\title{
Flexible Combinatorial Interaction Testing
}

\author{
Hanefi Mercan, Arsalan Javeed, and Cemal Yilmaz, Member, IEEE
}

\begin{abstract}
We present Flexible Combinatorial Interaction Testing (F-CIT), which aims to improve the flexibility of combinatorial interaction testing (CIT) by eliminating the necessity of developing specialized constructors for CIT problems that cannot be efficiently and effectively addressed by the existing CIT constructors. F-CIT expresses the entities to be covered and the space of valid test cases, from which the samples are drawn to obtain full coverage, as constraints. Computing an F-CIT object (i.e., a set of test cases obtaining full coverage under a given coverage criterion) then turns into an interesting constraint solving problem, which we call cov-CSP. cov-CSP aims to divide the constraints, each representing an entity to be covered, into a minimum number of satisfiable clusters, such that a solution for a cluster represents a test case and the collection of all the test cases generated (one per cluster) constitutes an F-CIT object, covering each required entity at least once. To solve the cov-CSP problem, thus to compute F-CIT objects, we first present two constructors. One of these constructors attempts to cover as many entities as possible in a cluster before generating a test case, whereas the other constructor generates a test case first and then marks all the entities accommodated by this test case as covered. We then use these constructors to evaluate F-CIT in three studies, each of which addresses a different CIT problem. In the first study, we develop structure-based F-CIT objects to obtain decision coverage-adequate test suites. In the second study, we develop order-based F-CIT objects, which enhance a number of existing order-based coverage criteria by taking the reachability constraints imposed by graph-based models directly into account when computing interaction test suites. In the third study, we develop usage-based F-CIT objects to address the scenarios, in which standard covering arrays are not desirable due to their sizes, by choosing the entities to be covered based on their usage statistics collected from the field. We also carry out user studies to further evaluate F-CIT. The results of these studies suggest that F-CIT is more flexible than the existing CIT approaches.
\end{abstract}

Keywords-Combinatorial interaction testing, covering arrays, sequence covering arrays, constraint solving, structural coverage, coverage criteria

\section{INTRODUCTION}

Exhaustively testing the input spaces of modern software systems in a timely manner (if not impossible at all) is generally far beyond the available resources for testing [1], such as time, computers, storage devices, network resources, and person-hour. Combinatorial interaction testing (CIT) approaches systematically sample the input space and test only the selected instances of the system's behavior [1], [2]. Note that the term "input" in CIT is used in the most general sense to refer to any factor, which can affect program executions, such as configuration options, input parameters, user events, etc.

CIT approaches typically model the software under test as a set of parameters, each of which takes its values from a discrete domain. As not all possible combinations of parameter values may be valid in practice, the model can also have a set of constraints, which invalidate certain combinations. Based on this model, CIT then generates a sample, i.e., a set of test cases, which from now on will be referred to as a CIT object, meeting a specified coverage criterion. That is, the sample contains some specified combinations

- H. Mercan, A. Javeed, and C. Yilmaz are with the Faculty of Engineering and Natural Sciences, Sabanci University, Istanbul, Turkey. E-mail: \{hanefimercan, ajaveed, cyilmaz\}@sabanciuniv.edu of parameters and their values. For instance, $t$-way covering arrays - a well-known CIT approach, where $t$ is called the coverage strength - requires that each valid combination of parameter values for every combination of $t$ parameters appears at least once in the sample [3], aiming to reveal all the failures caused by the interactions of $t$ or fewer parameters.

As an example, which will further be studied in detail in Section 2, Figure 1a presents a configurable system with 6 compile-time configuration options $\left(o_{1}, \ldots, o_{6}\right)$ implemented by using preprocessor directives. Each option has two levels of settings $\{(T) r u e$, (F)alse $\}$ and there are no inter-option constraints (i.e., all combinations of option settings are valid). The set of test cases in Figure $1 \mathrm{~b}$ represent a 2-way covering array, i.e., a CIT object, for this system. Since $t=2$, all pairwise combinations of settings for these 6 configuration options can be found in at least one of the 7 test cases selected by this CIT object.

To reduce the cost of testing, CIT constructors, i.e., the tools to compute CIT objects, aim to obtain a full coverage under the given criterion by using the smallest number of test cases possible. CIT has indeed been successfully used in many application domains, including systematic testing of network protocols [4], input parameters [5], software configurations [6], software product lines [7], multi-threaded applications [8], and graphical user interfaces [9]. 


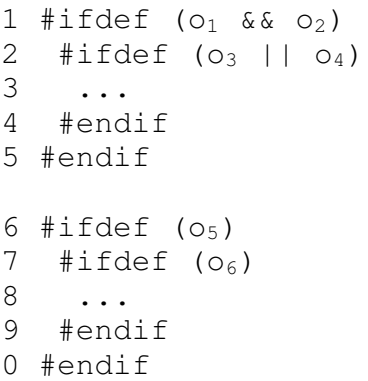

(a)

\begin{tabular}{|l|}
\hline entities to be covered \\
\hline$e_{1}:\left(o_{1} \wedge o_{2}\right)$ \\
$e_{2}: \neg\left(o_{1} \wedge o_{2}\right)$ \\
$e_{3}:\left(o_{1} \wedge o_{2}\right) \wedge\left(o_{3} \vee o_{4}\right)$ \\
$e_{4}:\left(o_{1} \wedge o_{2}\right) \wedge \neg\left(o_{3} \vee o_{4}\right)$ \\
$e_{5}:\left(o_{5}\right)$ \\
$e_{6}:\left(\neg O_{5}\right)$ \\
$e_{7}:\left(o_{5} \wedge o_{6}\right)$ \\
$e_{8}:\left(o_{5} \wedge \neg O_{6}\right)$ \\
\hline
\end{tabular}

(c)

\begin{tabular}{|cccccc|cccc|}
\hline \multicolumn{7}{|c|}{ test cases } & \multicolumn{5}{|c|}{ decision outcomes } \\
$o_{1}$ & $o_{2}$ & $o_{3}$ & $o_{4}$ & $o_{5}$ & $o_{6}$ & $o_{1} \wedge o_{2}$ & $o_{3} \vee o_{4}$ & $o_{5}$ & $o_{6}$ \\
\hline $\mathrm{T}$ & $\mathrm{F}$ & $\mathrm{T}$ & $\mathrm{F}$ & $\mathrm{F}$ & $\mathrm{F}$ & $F$ & - & $\mathrm{F}$ & - \\
$\mathrm{F}$ & $\mathrm{T}$ & $\mathrm{F}$ & $\mathrm{T}$ & $\mathrm{T}$ & $\mathrm{F}$ & $\mathrm{F}$ & - & $\mathrm{T}$ & $\mathrm{F}$ \\
$\mathrm{T}$ & $\mathrm{T}$ & $\mathrm{T}$ & $\mathrm{T}$ & $\mathrm{F}$ & $\mathrm{T}$ & $\mathrm{T}$ & $\mathrm{T}$ & $\mathrm{F}$ & - \\
$\mathrm{T}$ & $\mathrm{F}$ & $\mathrm{F}$ & $\mathrm{F}$ & $\mathrm{T}$ & $\mathrm{F}$ & $\mathrm{F}$ & - & $\mathrm{T}$ & $\mathrm{F}$ \\
$\mathrm{F}$ & $\mathrm{F}$ & $\mathrm{T}$ & $\mathrm{F}$ & $\mathrm{T}$ & $\mathrm{T}$ & $\mathrm{F}$ & - & $\mathrm{T}$ & $\mathrm{T}$ \\
$\mathrm{F}$ & $\mathrm{F}$ & $\mathrm{F}$ & $\mathrm{T}$ & $\mathrm{F}$ & $\mathrm{T}$ & $\mathrm{F}$ & - & $\mathrm{F}$ & - \\
$\mathrm{T}$ & $\mathrm{T}$ & $\mathrm{T}$ & $\mathrm{F}$ & $\mathrm{F}$ & $\mathrm{T}$ & $\mathrm{T}$ & $\mathrm{T}$ & $\mathrm{F}$ & - \\
\hline
\end{tabular}

(b)

\begin{tabular}{|cccccc|ccccc|}
\hline \multicolumn{7}{|c|}{ test cases } & \multicolumn{4}{c|}{ decision outcomes } \\
$o_{1}$ & $o_{2}$ & $o_{3}$ & $o_{4}$ & $o_{5}$ & $o_{6}$ & $o_{1} \wedge o_{2}$ & $o_{3} \vee o_{4}$ & $o_{5}$ & $o_{6}$ \\
\hline $\mathrm{T}$ & $\mathrm{T}$ & $\mathrm{T}$ & $\mathrm{T}$ & $\mathrm{T}$ & $\mathrm{T}$ & $T$ & $T$ & $T$ & $T$ \\
$\mathrm{~F}$ & $\mathrm{~F}$ & $\mathrm{~T}$ & $\mathrm{~F}$ & $\mathrm{~F}$ & $\mathrm{~T}$ & $F$ & - & $F$ & $T$ \\
$\mathrm{~T}$ & $\mathrm{~T}$ & $\mathrm{~F}$ & $\mathrm{~F}$ & $\mathrm{~T}$ & $\mathrm{~F}$ & $T$ & $F$ & $T$ & $F$ \\
\hline
\end{tabular}

(d)

Fig. 1: (a) An example set of preprocessor directives for a system with 6 compile-time configuration options, (b) an example 2-way standard covering array created for the system, (c) entities to be covered to obtain full coverage under the decision coverage criterion, and (d) an example test suite obtaining full coverage under the decision coverage criterion.

We, however, observe that when the actual CIT problems differ from the ones addressed by the existing CIT approaches, it can be difficult to use these approaches in an efficient and effective manner [1], [10], [11]. Note that, in this context, changes in CIT problems refer to changes in the coverage criteria or in the properties of the test spaces from which the samples are drawn, such that existing CIT constructors cannot be used as they are (i.e., requiring modifications, if at all possible) or demand excessive number of test cases to guarantee full coverage.

For example, if the coverage criterion in our running example was changed from $t$-way coverage to decision coverage [12], where the goal is to cover every outcome of a decision in Figure 1a at least once, then, to guarantee full coverage, the strength of the standard covering array to be used would be at least 4 (i.e., $t \geq 4)$. This is because the outcome of the decision in line 2 (Figure 1a) depends on the interactions among 4 options, namely $o_{1}, o_{2}, o_{3}$, and $o_{4}$. This, however, requires to have at least 16 test cases, while a full decision coverage in this scenario can be achieved by using as little as 3 test cases, such as the ones given in Figure $1 \mathrm{~d}$.

Different CIT problems typically necessitate the development of specialized constructors. Taking a brief look at the historical perspective of covering arrays can help understand this trend: The very first variants of covering array constructors supported only pairwise testing of binary parameters, where $t=2$ and each parameter had exactly two levels of values [13]. When these strict conditions were not met, the aforementioned objects were of little worth. Consequently, new CIT constructors were developed to handle the CIT problems, in which the parameters could take on a different number of values and the covering arrays could be computed for $t \geq 2$ [3]. However, as these objects assumed that all possible combinations of parameter values were valid, they were not appropriate in the presence of system-wide inter-parameter constraints, causing wasted resources in testing [14], [15]. Thus, new CIT constructors were developed to handle system-wide constraints [16], [17]. However, these objects then became inappropriate in the presence of test case-specific constraints, which led to the development of test case-aware covering arrays and their specialized constructors [18].

Developing specialized constructors can, however, be quite challenging and time-consuming, which is also apparent from more than 50 papers published in the literature, the sole purpose of which is to compute standard covering arrays [1], [2].

In this work, we introduce Flexible Combinatorial Interaction Testing (F-CIT) to improve the flexibility of CIT by eliminating the necessity of developing specialized constructors for every distinct CIT problem. In F-CIT, 
both the entities to be covered and the space of test cases, from which the samples will be drawn, are expressed as constraints. The problem of computing an F-CIT object to cover all the requested entities then turns into an interesting constraint solving problem, which we call cov-CSP [19], [20], [21]. Given a set of constraints, each of which represents an entity to be covered, cov-CSP aims to divide the constraints into a minimum number of satisfiable clusters, such that each cluster depicts a subset of the entities, which can be tested together in a single test case. A solution for a cluster then represents a test case, covering all the entities included in the cluster. Consequently, the collection of all the test cases generated (one per cluster) constitutes an F-CIT object that covers each required entity at least once. In the remainder of the paper, we use the terms "CIT object" and "F-CIT object" interchangeably to refer to a set of test cases, which obtain full coverage under a given coverage criterion.

Going back to our running example (Figure 1), a decision coverage-adequate F-CIT object can be computed by representing each configuration option as a Boolean variable. Then, each entity to be covered corresponds to a distinct outcome of a decision, represented as a constraint in Boolean logic. Figure 1c presents all the entities that need to be covered to obtain full decision coverage for the system given in Figure 1 a. These entities can be divided into 3 satisfiable clusters: $\left\{e_{1}, e_{3}, e_{5}, e_{7}\right\},\left\{e_{2}, e_{6}\right\}$, and $\left\{e_{4}, e_{8}\right\}$. A solution for each cluster represents a test case. For example, the three test cases in Figure 1d, each of which corresponds to a solution computed for a distinct cluster, represent an F-CIT object, achieving full decision coverage.

Note that we use the term "constraint" in the general sense in F-CIT. That is, any restriction, independent of the logic in which it is specified, is considered to be a constraint. Consequently, an F-CIT constructor can be used as long as the entities to be covered are expressed as constraints and an appropriate procedure (i.e., a "solver") is provided to determine if a given set of entities can be tested together in a single test case, i.e., if the respective constraints can be satisfied together. In our running example (Figure 1), for instance, we can use an ordinary SAT or CSP solver [22] to figure out whether the constraints included in the clusters are satisfiable or not. We, therefore, believe that F-CIT can be used in a wide spectrum of domains, including software product lines, system of systems, and cyberphysical systems, in addition to the domains, which we used for evaluating F-CIT in this work, i.e., highlyconfigurable systems and event-driven systems.

Note further that using constraint solving techniques for combinatorial interaction testing is not a new idea [6], [10], [15], [16], [17], [23], [24]. However, the constraints in F-CIT are interpreted quite differently than the ones used in existing CIT approaches. More specifically, while the constraints in existing CIT approaches are typically used to specify combinations of parameter values that should be avoided, they are used in F-CIT to specify both the combinations (i.e., the entities) to be covered and the space of valid test cases, from which the samples are drawn. Therefore, the scope of a constraint in existing CIT approaches is all the test cases included in a covering array. That is, all of the selected test cases should satisfy all the constraints. On the other hand, the scope of a constraint representing an entity to be covered in F-CIT is limited to a single test case. That is, such a constraint needs to be satisfied by at least one test case, rather than by all the test cases selected, allowing a considerable amount of flexibility.

For instance, in our running example (Figure 1), expressing $O_{5}$ and $\neg O_{5}$ (i.e., the outcomes of the decision in line 6) as constraints to selectively determine what to cover in standard covering arrays, prevents the generation of any covering arrays as these conflicting constraints are enforced to be satisfied by all of the selected test cases. In F-CIT, however, these constraints are required to be satisfied by different test cases. For example, in Figure 1d, while the former constraint is satisfied by the first and third test cases, the latter one is satisfied by the second test case.

F-CIT is not a methodology for deciding what needs to be tested. It, in fact, takes as input a set of entities to be covered and aims to cover them in a minimum number of test cases by accommodating as many entities as possible in a single test case. Note that for a given CIT problem, regardless of whether an F-CIT constructor is to be used or a specialized constructor is to be developed, entities to be covered need to be enumerated and a procedure needs to be devised to determine whether a given set of entities can be covered together in a single test case or not. Once these are given, though, F-CIT provides a constructor right away.

Furthermore, F-CIT does not aim to replace existing CIT approaches. We, indeed, don't see much value in using F-CIT to compute the same CIT objects that the existing CIT constructors compute, as the generalized F-CIT constructors may not be as efficient and as effective as their specialized counterparts. We rather aim to reduce the barriers to applying CIT to other domains and testing problems by generalizing the construction of CIT objects as much as possible, so that the collective effort spent for developing FCIT constructors can be leveraged to address a wider spectrum of CIT problems.

In this work, we present two F-CIT constructors, namely cover-and-generate and generate-and-cover. While the former aims to cover as many entities as possible in a cluster first and then generates a test case for the cluster, the latter generates a test case first and then 
marks all the entities accommodated by the test case as covered.

To evaluate F-CIT, we then carry out three case studies, each of which focuses on a different CIT problem. In the first study, we use F-CIT to compute structural code coverage-based test suites. In the second study, we use F-CIT to improve a number of existing order-based covering arrays for testing event-driven systems by taking the reachability constraints imposed by graph-based models directly into account during the construction of CIT objects. In the last study, we use F-CIT to compute usage-based CIT objects, where the entities to be covered are determined according to their usage statistics in the field - an approach which is of importance especially when standard covering arrays are not desirable due to their sizes.

In these studies, we observed that it was either unclear how to use the existing constructors (if at all possible) to compute the requested CIT objects; or the existing constructors required non-trivial modifications or excessive number of test cases to guarantee a full coverage. F-CIT, on the other hand, used the same constructor to compute all the requested CIT objects without requiring any modifications, demonstrating the flexibility of the proposed approach.

We also carry out user studies to further evaluate the proposed approach. More specifically, we observe human subjects working on the smaller instances of the very same CIT problems we study in this work and report the results we obtained together with the insights we gained.

In previous work [25], we presented an initial set of definitions for F-CIT and provided an algorithm for computing F-CIT objects. And, we did this without providing any implementations or empirical evaluations. In this work, however, we present a simplified set of more formal definitions, an additional F-CIT constructor, a tool implementing the F-CIT constructors, and three case studies together with user studies, in which F-CIT is evaluated.

The contributions of this work can be summarized as follows:

- A flexible approach, F-CIT, for computing combinatorial objects for testing,

- Two constructors together with a tool implementing these constructors to compute F-CIT objects,

- Definition and construction of structure-based FCIT objects,

- Definition and construction of order-based F-CIT objects,

- Definition and construction of usage-based F-CIT objects,

- A series of experiments demonstrating the flexibility of F-CIT,

- User studies demonstrating the usability of F-CIT.
The remainder of the paper is organized as follows: Section 2 provides a motivating example; Section 3 introduces F-CIT; Section 4 develops two constructors for computing F-CIT objects; Section 5 presents three case studies, demonstrating the drawbacks of the existing CIT approaches and how F-CIT addresses these drawbacks; Section 6 presents the user studies; Section 7 provides a general discussion of the applicability of F-CIT; Section 8 discusses threats to validity; Section 9 discusses related work; and Section 10 presents concluding remarks and possible directions for future work.

\section{Motivating Example}

In this section, we provide more details on our running example used in Section 1. In this example, we are concerned with compile-time configuration options implemented in the form of preprocessor directives, such as \#ifdef and \#ifndef directives found in $\mathrm{C}$ and $\mathrm{C}++$. Figure 1a presents a hypothetical system with 6 compile-time configuration options, namely $o_{1}, \ldots, o_{6}$, each of which happens to have two levels of settings (T)rue and (F)alse. In the remainder of the paper, we use the term "if-then-else directive" to refer to an \#ifdef, \# ifndef, or a similar conditional branch directive, the conditions of which are comprised of only compiletime configuration options and/or constants. Note that such directives allow the decision outcomes to be directly controlled from outside the system by modifying the settings of the compile-time options as a part of the build process.

An if-then-else directive essentially describes how configuration options interact with each other. That is, the outcome of a decision (thus the behavior of the system) may change due to these interactions. Consequently, these interactions may need to be tested. To this end, one structural test adequacy criterion that the developers can use is the decision coverage (DC) criterion. A full coverage under DC is obtained when every decision, such as $\left(o_{1} \wedge o_{2}\right)$ and $\left(o_{3} \vee o_{4}\right)$ in Figure 1a, is evaluated to both true and false.

Consider a scenario where the goal is to create a DCadequate test suite for the system given in Figure 1a. Note that since a single configuration can cover multiple decision outcomes, the number of configurations required to obtain full coverage under DC can be reduced by covering as many outcomes as possible in each of the selected configurations. This is, indeed, the main motivation behind CIT. Therefore, CIT should be of help.

\subsection{Applying standard CIT}

It, however, turns out that standard covering arrays are infeasible to achieve the aforementioned coverage criterion in an efficient and effective manner. 
As an in initial attempt, a standard 2-way covering given in Figure $1 \mathrm{~b}$ is created. The first 6 columns in this figure present the 2-way covering array and the last 4 columns depict the outcomes of the decisions: ' $T$ ' for true, ' $F$ ' for false, and ' - ' for decisions that are not exercised due to some unsatisfied guard conditions. For example, the first row indicates that the decision $\left(o_{3} \vee o_{4}\right)$ is not exercised by the configuration $\left(o_{1}=T, o_{2}=F, o_{3}=T, o_{4}=F, o_{5}=F, o_{6}=F\right)$, because the guard condition $\left(o_{1} \wedge o_{2}\right)$ evaluates to $F$.

This covering array while obtaining a full coverage for the if-then-else directive between the lines 6 and 10 in Figure 1a, obtains only $75 \%$ DC coverage for the ifthen-else directive between the lines 1 and 5 , covering 3 out of 4 decision outcomes required for full coverage. More specifically, out of the decision outcomes $\left\{\left(o_{1} \wedge\right.\right.$ $\left.\left.o_{2}\right), \neg\left(o_{1} \wedge o_{2}\right),\left(o_{1} \wedge o_{2}\right) \wedge\left(o_{3} \vee o_{4}\right),\left(o_{1} \wedge o_{2}\right) \wedge \neg\left(o_{3} \vee o_{4}\right)\right\}$, the last one where the inner decision $\left(o_{3} \vee o_{4}\right)$ needs to be evaluated to $F$, is not covered. Note that this outcome can only be achieved with a single 4 -way combination, in which $o_{1}=T, o_{2}=T, o_{3}=F$, and $o_{4}=F$.

One solution approach to overcome this issue is to increase the strength of the covering array, i.e., to use a larger $t$. This, however, can excessively increase the number of configurations to be tested. For example, since the missing combination in our example is a 4way combination, to guarantee the coverage of this combination, a 4-way covering array needs to be created at the very least. However, a 4 -way covering array for this scenario can have as many as 28 configurations.

An alternative approach is to use a variable-strength covering array, requiring a 4-way coverage only for the options $\left\{o_{1}, \ldots, o_{4}\right\}$. However, since what is actually being requested is the exhaustive testing of all possible combinations of settings for these 4 binary options, at least 16 configurations are required by this alternative.

Note that decision outcomes that need to be covered cannot be expressed as constraints in standard covering arrays in an attempt to selectively determine what to cover. This is because constraints in standard covering arrays are globally enforced. That is, the constraints should be satisfied by each and every configuration included in the covering array. Therefore, expressing the decision outcomes as constraints in standard covering arrays prevents the creation of any CIT objects because the alternative outcomes of a decision are guaranteed to conflict with each other. For example, since the outcomes of the decision at line 6 in Figure 1a, i.e., $O_{5}$ and $\neg O_{5}$, conflict with each other, no configuration satisfying both of these constraints can be generated; thus, no standard covering array can be constructed.

\subsection{Applying F-CIT}

F-CIT, on the other hand, can flexibly be used as follows to obtain DC-adequate test suites. Each entity to be covered corresponds to a distinct decision outcome. The entities are then expressed as constraints by using Boolean logic where each configuration option is represented by a Boolean variable. For our running example, Figure 1c presents all the entities required to be covered to obtain full coverage under the DC criterion. For instance, the first two entities $\left(e_{1}\right.$ and $\left.e_{2}\right)$ represent the $T$ and $F$ outcomes of the decision at line 1 in Figure 1a, respectively.

Given the entities in Figure 1c, an F-CIT constructor divides them into 3 clusters: $\left\{e_{1}, e_{3}, e_{5}, e_{7}\right\},\left\{e_{2}, e_{6}\right\}$, and $\left\{e_{4}, e_{8}\right\}$, such that all the constraints within a cluster can be satisfied together and that the number of clusters required to cover all the entities is minimized as much as possible.

Each cluster represents a set of decision outcomes that can be covered together in a single configuration. Therefore, a solution computed for a cluster represents a configuration, which covers all the decision outcomes included in the cluster. Consequently, the FCIT constructor generates the three configurations (one for each cluster) given in Figure 1d, which obtain full coverage under the DC criterion; the first configuration covers the entities $\left\{e_{1}, e_{3}, e_{5}, e_{7}\right\}$, the second configuration covers $\left\{e_{2}, e_{6}\right\}$, and last configuration covers $\left\{e_{4}, e_{8}\right\}$.

Note that neither the clusters nor the configurations generated in this study are unique in the sense that there are other sets of configurations that an F-CIT constructor can generate to achieve full coverage. This is indeed similar to what we have in standard covering arrays as different $t$-way covering arrays can be computed for the same input space model.

Note further that although half of the constraints in Figure 1c conflict with the other half, it does not create an issue for F-CIT. This is because as each of these constraints represents an entity to be covered, F-CIT enforces them at the level of a test case. This, in turn, improves the flexibility of CIT, compared to enforcing the constraints at the level of a test suite as is the case with standard covering arrays where each and every test case included in a test suite should satisfy all the constraints. That is, F-CIT aims to satisfy each constraint representing an entity in at least one test case, rather than enforcing all the selected test cases to satisfy all of the entity constraints. For example, in the test suite given in Figure 1d, the constraint for entity $e_{2}: \neg\left(o_{1} \wedge o_{2}\right)$ is satisfied by the second configuration only. The other configurations included in this test suite, indeed, violate this constraint.

\section{F-CIT}

F-CIT takes as input a set of entities $E$ to be covered and a model $M=\langle P, D, C\rangle$, where $P=\left\{p_{1}, p_{2}, \ldots, p_{k}\right\} \quad$ is a set of parameters, $D=\left\{D_{1}, D_{2}, \ldots, D_{k}\right\}$ is a set of respective domains 
of values, and $C$ is a constraint defined over $P$. While $C$ defines the space of valid test cases, from which the samples are drawn, $E$ specifies what needs to be covered by these samples.

Next, we make a number of definitions, starting from the "standard" definitions and going towards the FCIT-specific ones:

Definition 1. A constraint is a tuple $\left\langle P^{\prime}, R\right\rangle$ where $P^{\prime} \subseteq P$ is a subset of $l \leq k$ parameters and $R$ is an l-ary relation on the corresponding domains.

Definition 2. An evaluation is a function from a subset of parameters to a particular set of values in the corresponding subset of domains.

Definition 3. An evaluation satisfies a constraint $<P^{\prime}, R>$, if the values assigned to the parameters in $P^{\prime}$, satisfies the relation $R$.

Definition 4. An evaluation is consistent with respect to a set of constraints, if it satisfies all the constraints.

Definition 5. An evaluation is complete, if it includes all the parameters in $P$.

Definition 6. An F-CIT testable entity is a constraint over a subset of $P$, which has at least one evaluation consistent with $C$, representing an entity to be covered in testing.

Definition 7. An F-CIT test case is a complete evaluation of $P$, which is consistent with $C$.

Definition 8. An F-CIT testable entity is said to be covered by an F-CIT test case, if and only if the test case is consistent with the testable entity.

Definition 9. Given an F-CIT model $M=\langle P, D, C\rangle$ and a set of testable entities $E$ to be covered, an F-CIT object is a set of F-CIT test cases, such that every F-CIT testable entity in E, is covered by at least one F-CIT test case.

Going back to our running example in Section 2, the F-CIT model $M=\langle P, D, C\rangle$ is defined as follows: $P=\left\{o_{1}, \ldots, o_{6}\right\}$, $D=\{\{T, F\},\{T, F\},\{T, F\},\{T, F\},\{T, F\},\{T, F\}\}$, and $C$ : true, indicating that all possible configurations are valid. An F-CIT testable entity corresponds to a distinct decision outcome expressed as a constraint in Boolean logic. The testable entities to be covered $E=\left\{e_{1}, \ldots, e_{8}\right\}$ are then defined as they are given in Figure 1c. For example, the testable entity $e_{1}$ is defined as $\neg\left(o_{1} \wedge o_{2}\right)$, representing the $F$ outcome of the first decision in Figure 1a. An F-CIT test case corresponds to a configuration, in which each configuration option assumes the value of either $T$ or $F$, such as the second configuration in Figure $1 \mathrm{~d}$ where $o_{1}=F, o_{2}=F$, $o_{3}=T, o_{4}=F, o_{5}=F$, and $o_{6}=T$. An F-CIT object then corresponds to a decision coverage-adequate set of F-CIT test cases, such as the ones given in Figure 1d.

\section{Computing F-CIT OBJects}

It turns out that computing F-CIT objects requires us to solve an interesting constraint satisfaction problem, which we call cov-CSP, inspired from the theoretical concepts for "measuring" the level of consistency in paraconsistent logic (i.e., "inconsistency-tolerant" systems of logic) [19], [20], [21].

Given a set of constraints $H$, cov-CSP aims to divide $H$ into a minimum number of satisfiable clusters. That is, cov-CSP seeks to satisfy the constraints, not necessarily as a whole, but in groups. We first define cov-CSP in the most general sense and then show how solving this problem helps compute F-CIT objects.

Definition 10. Given a set of constraints $H=\left\{h_{1}, \ldots, h_{m}\right\}$, cov-CSP divides $H$ into a minimum number of clusters $S=\left\{H_{1}, \ldots, H_{n}\right\}$, such that $\bigcup_{H_{i} \in S} H_{i}=H$ and that for each $H_{i} \in S, \bigwedge_{h \in H_{i}} h$ is satisfiable, i.e., all the constraints in a cluster are satisfiable together.

Given a model $M=<P, D, C>$ and a set of F-CIT testable entities to be covered $E=\left\{e_{1}, \ldots, e_{m}\right\}$, each of which is represented as a constraint, computing an FCIT object proceeds by first solving the cov-CSP problem, so that $E$ is divided into a "minimum" number of satisfiable clusters $S=\left\{E_{1}, \ldots, E_{n}\right\}$ (as specified by Definition 10). Note that since computing the global minimum may not be computationally feasible (or desirable), F-CIT aims to compute an approximation to it.

Each cluster depicts a set of testable entities that can be tested together. Therefore, a solution for a cluster, represents an F-CIT test case covering all the F-CIT testable entities included in the cluster. Consequently, the collection of all the test cases generated (one per cluster), constitutes an F-CIT object covering each testable entity in $E$ at least once.

The only remaining detail to ensure the generation of valid test cases, is to take the model constraint $C$ into account. To this end, when checking the satisfiability of a cluster $E_{i} \in S$ or computing a solution for it, the constraint to satisfy simply becomes $C \wedge \bigwedge_{e \in E_{i}} e$.

Note that, in order to reduce the number of test cases required, it is desirable to avoid redundancy as much as possible by covering each testable entity in exactly one test case. However, a testable entity, in the process of covering other testable entities, may end up being covered by multiple test cases. This can happen unintentionally (i.e., by chance) or intentionally to satisfy the model constraint $C$.

Next, we present two constructors for computing FCIT objects (thus, for solving the cov-CSP problem), namely cover-and-generate and generate-and-cover. 


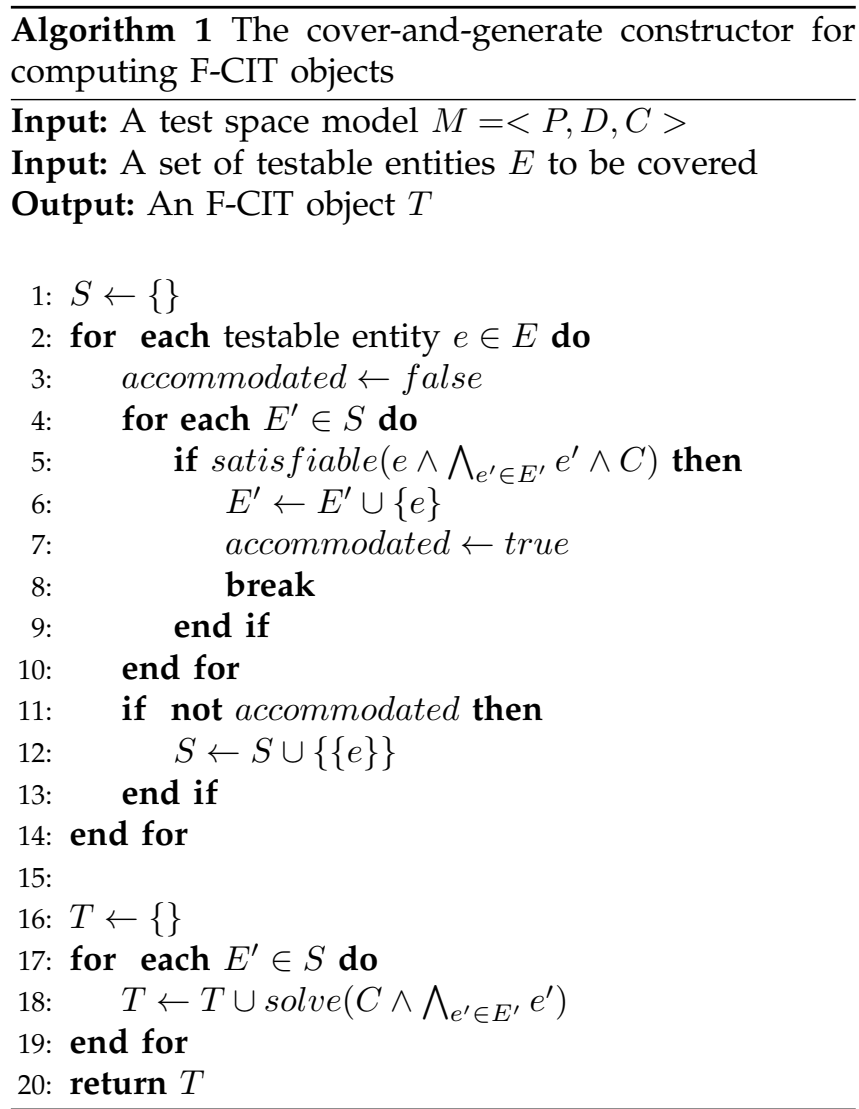

\subsection{The Cover-and-Generate Constructor}

The cover-and-generate constructor (Algorithm 1) maintains a pool $S$ of clusters, each representing a set of testable entities that can be covered together. The pool is initially empty (line 1). Then, for each testable entity $e \in E$, we attempt to accommodate it in an existing cluster $E^{\prime} \in S$ (lines 4-10). To this end, we check to see if $e$ is satisfiable together with all the constraints in $E^{\prime}$ as well as with the model constraint $C$, i.e., whether $e \wedge \bigwedge_{e^{\prime} \in E^{\prime}} e^{\prime} \wedge C$ is satisfiable (line 5). If so, $e$ is included in $E^{\prime}$ (line 6), indicating that $e$ can be accommodated together in a single test case with the other testable entities in $E^{\prime}$. Otherwise (i.e., if no such cluster is found), we populate $S$ with a new cluster initially having only $e$ (line 12). Once all the testable entities are processed, for each cluster $E^{\prime} \in S$, we generate a test case by solving $C \wedge \bigwedge_{e^{\prime} \in E^{\prime}} e^{\prime}$ (line $18)$. The collection of all the test cases generated $(T)$, is then returned as the F-CIT object computed, covering all the testable entities in $E$ (lines 17-20).

\subsection{The Generate-and-Cover Constructor}

The generate-and-cover constructor associates a cluster with an F-CIT test case, rather than with a set of F-CIT testable entities. Conceptually, this constructor generates a test case first and then marks all the testable entities accommodated by the test case as covered.
Algorithm 2 The generate-and-cover constructor for computing F-CIT objects

Input: A test space model $M=\langle P, D, C>$ Input: A set of testable entities $E$ to be covered Output: An F-CIT object $T$

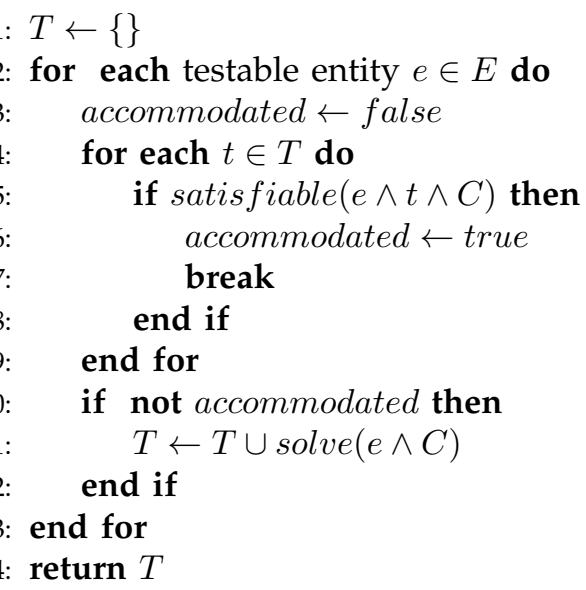

Therefore, it is different than the cover-and-generate constructor, which attempts to cover as many testable entities as possible in a cluster before generating a test case. Consequently, the set of clusters maintained through the iterations of the generate-and-cover constructor, simply represents the F-CIT test cases that have already been included in the F-CIT object being computed.

Given a model $M=<P, D, C>$ and a set of testable entities $E$ to be covered, one way to generate a test case is to compute a solution for the model constraint $C$, regardless of $E$. However, generating test cases without taking the testable entities to be covered into account, may make it quite difficult to cover the entities that are hard to cover by chance. We, therefore, employ an alternative approach in this work, which guarantees that at least one previously uncovered testable entity is covered by every test case generated.

Algorithm 2 presents the generate-and-cover constructor. The F-CIT object $T$ is initially empty (line 1 ). Then, for each testable entity $e \in E$, we check to see if $e$ has already been covered by a test case $t \in T$ (lines 4-9), i.e., if there exists a test case $t \in T$, which is consistent with $e$ (line 5). If no such test case is found, a new F-CIT test case covering $e$, is generated by solving the constraint $e \wedge C$ and $T$ is populated with the newly generated test case (lines 10-12). Once all the testable entities in $E$ have been processed, $T$ is returned as the F-CIT object computed (line 14).

\subsection{A Seeding Mechanism}

Both of the constructors we have discussed so far can also take as input a seed, which in this context refers 
to a set of F-CIT test cases. Given a seed, all the FCIT testable entities in the seed, are considered to have already been covered and additional F-CIT test cases are generated only to cover the remaining entities.

To this end, the only change that needs to be made is to modify line 1 in Algorithms 1 and 2, such that instead of starting with an empty pool of clusters, we start with an initially populated pool of clusters, each of which is created to include a single F-CIT test case in the seed. Nothing else in the algorithms needs to be changed.

In Section 5.1, we use the seeding mechanism both to compute higher strength F-CIT objects from lower strength F-CIT objects (by using the lower strength objects as seeds) and to generate F-CIT objects that satisfy multiple coverage criteria (by using an object satisfying a coverage criterion as a seed to compute another object satisfying a different coverage criterion).

\subsection{Example: Computing DC-Adequate Test Suites as F-CIT Objects}

In this section, for illustrative purposes, we use the cover-and-generate constructor (Algorithm 1) to compute DC-Adequate test suites as F-CIT objects using our running example in Section 2. For the sake of the discussion, however, we introduce the following system-wide constraint to the problem: $\left(o_{2}=F\right) \Longrightarrow\left(o_{6}=T\right)$, i.e., if $o_{2}$ is false, then $o_{6}$ must be true, invalidating the combination $\left(o_{2}=F, o_{6}=F\right)$.

Modeling. The F-CIT model is defined as $\quad M=\langle P, D, C\rangle$, where $P=\left\{o_{1}, \ldots, o_{6}\right\}$, $D=\{\{T, F\}, \ldots,\{T, F\}\}$, and $C:\left(\neg O_{2} \Longrightarrow o_{6}\right)$. Each F-CIT testable entity then naturally corresponds to a decision outcome to be covered. Figure 1c presents all the F-CIT testable entities that need to be covered to obtain full coverage under the decision coverage criterion.

Assuming that the testable entities in Figure 1c are processed in the order $e_{1}, \ldots, e_{8}$, the coverand-generate constructor proceeds as follows: First, $e_{1}:\left(o_{1} \wedge o_{2}\right)$ is processed. Since the pool $S$ is initially empty (line 1), a new cluster $E_{1}=\left\{e_{1}\right\}$ is created and $S$ is populated with $E_{1}$, i.e., $S=\left\{E_{1}\right\}$ (line 12). Then, $e_{2}: \neg\left(o_{1} \wedge o_{2}\right)$ is processed. Since $e_{1} \wedge e_{2} \wedge C$, i.e., $\left(o_{1} \wedge o_{2}\right) \wedge \neg\left(o_{1} \wedge o_{2}\right) \wedge\left(\neg o_{2} \Longrightarrow o_{6}\right)$, is not satisfiable (line 5), $e_{2}$ cannot be placed in $E_{1}$. So, a new cluster $E_{2}=\left\{e_{2}\right\}$ is created and $S$ is updated to $\left\{E_{1}, E_{2}\right\}$ (line 12). Next, $e_{3}:\left(o_{1} \wedge o_{2}\right) \wedge\left(o_{3} \vee o_{4}\right)$ is processed. Since $e_{1} \wedge e_{3} \wedge C$, i.e., $\left(o_{1} \wedge o_{2}\right) \wedge\left(\left(o_{1} \wedge o_{2}\right) \wedge\left(o_{3} \vee o_{4}\right)\right) \wedge\left(\neg o_{2} \Longrightarrow o_{6}\right)$, is satisfiable (line 5), $e_{3}$ is included in $E_{1}$ (line 6). After processing all the remaining testable entities in Figure $1 \mathrm{c}$, we have the clusters given in the first column of Table 1.

For each cluster in $S=\left\{E_{1}, E_{2}, E_{3}\right\}$, we then generate an F-CIT test case by satisfying the
TABLE 1: An F-CIT object (second column) created for the set of satisfiable clusters $S=\left\{E_{1}, E_{2}, E_{3}\right\}$ (first column) obtained for the testable entities in Figure 1c.

\begin{tabular}{|c|cccccc|}
\hline satisfiable clusters & \multicolumn{7}{|c|}{ DC-adequate } & F-CIT object \\
$S=\left\{E_{1}, E_{2}, E_{3}\right\}$ & $o_{1}$ & $o_{2}$ & $o_{3}$ & $o_{4}$ & $o_{5}$ & $o_{6}$ \\
\hline$E_{1}=\left\{e_{1}, e_{3}, e_{5}, e_{7}\right\}$ & $\mathrm{T}$ & $\mathrm{T}$ & $\mathrm{T}$ & $\mathrm{T}$ & $\mathrm{T}$ & $\mathrm{T}$ \\
$E_{2}=\left\{e_{2}, e_{6}\right\}$ & $\mathrm{F}$ & $\mathrm{F}$ & $\mathrm{T}$ & $\mathrm{F}$ & $\mathrm{F}$ & $\mathrm{T}$ \\
$E_{3}=\left\{e_{4}, e_{8}\right\}$ & $\mathrm{T}$ & $\mathrm{T}$ & $\mathrm{F}$ & $\mathrm{F}$ & $\mathrm{T}$ & $\mathrm{F}$ \\
\hline
\end{tabular}

constraints included in the cluster together with the model constraint $C$ (lines 16-19). For example, for $E_{1}$, solving $e_{1} \wedge e_{3} \wedge e_{5} \wedge e_{7} \wedge C$ produces the test case $\quad\left(o_{1}=T, o_{2}=T, o_{3}=T, o_{4}=T, o_{5}=T, o_{6}=T\right)$. Processing all the clusters would then generate the F-CIT object given in the second column of Table 1 (line 20), which is, indeed, DC-adequate.

\subsection{Discussion}

Regarding constraints and solvers. The terms "constraint" and "solver" are used in the general sense in F-CIT. That is, any restriction, independent of the logic in which it is specified, is considered to be a constraint and a solver conceptually determines whether a given set of testable entities can be covered together in a single test case or not. Therefore, F-CIT expects that the underlying solver supports essentially a single computational primitive, namely solve. The other primitive used in Algorithms 1 and 2, namely satisfiable, can actually be implemented by using solve as the absence of a solution indicates unsatisfiability.

Having a simple interface between F-CIT constructors and solvers further improves the flexibility of FCIT. For example, all of the widely-used SAT and CSP solvers, in one form or another, provide a solve primitive. Furthermore, this feature also allows applicationand domain-specific solvers to be used with F-CIT constructors (Section 5.3).

This interface can indeed be further generalized by having solve to take as input a set of constraints, each of which can represent a testable entity, a model constraint, or a test case. Since an F-CIT constructor does not then need to interpret these constraints, the testable entities, the model constraints, and the test cases can be expressed in any form desired, which may not even need to be formal.

Regarding constructors. We have presented two constructors in this section, namely the cover-andgenerate constructor and the generate-and-cover constructor. We introduced the latter solely to mimic one of the simplest ways of generating F-CIT objects: Keep on generating valid test cases until all the required entities have been covered. As such, we use this constructor as a base line for comparisons in our experiments (Section 5), demonstrating that computing F-CIT objects in an efficient and effective manner is not trivial. Indeed, 
the results of our experiments strongly suggest that the cover-and-generate constructor performed better than the generate-and-cover constructor in reducing both the sizes and the construction times of F-CIT objects (Section 5).

We, therefore, generally suggest to use the coverand-generate constructor. However, the generate-andcover constructor can still be of practical interest in scenarios especially when it is costly to determine whether multiple testable entities can be covered together or not (due to, for example, the complexity of the constraints to be solved) and when it is easy to cover the entities by chance in valid test cases. Note that the presence of these factors favors the generate-andcover constructor as multiple testable entities can be covered by generating a valid test case. Furthermore, by making sure that each test case covers at least one previously uncovered testable entity, the generate-andcover constructor guarantees the convergence into full coverage. Clearly, the end-users can always experiment with both constructors to determine the one to use in their projects.

With all these in mind, we have implemented the FCIT constructors given in Algorithms 1 and 2 in Python in the form of an extensible tool that can work with any types of constraints and solvers. The tool can be downloaded at https://github.com/susoftgroup/UCIT/.

The efficiency and effectiveness of the F-CIT constructors we introduced in this work (i.e., the construction times and the sizes of the F-CIT objects computed), can be effected by the order, in which the testable entities are processed. In the presence of some knowledge regarding a favorable order (or a partial order), the testable entities can be sorted accordingly before they are fed to an F-CIT constructor. If not, a random order can be used by shuffling the entities. Furthermore, the construction process can be repeated multiple times in an attempt to compute smaller F-CIT objects at the cost of increased construction times. In Section 5.3.4, we carry out additional set of experiments to evaluate the sensitivity of the cover-and-generate constructor (which generally performed better than the generateand-cover constructor) to the order the testable entities are processed.

Furthermore, F-CIT constructors may not be as efficient as their specialized counterparts. Our ultimate goal, however, is not to perform better than the existing constructors when F-CIT is used to compute the same CIT objects that these constructors are specifically designed to compute. As a matter of fact, we don't see much value in using F-CIT in such scenarios unless the F-CIT constructors perform better than the existing ones. Our goal is rather to improve the flexibility, thus the applicability, of CIT by eliminating the necessity of developing specialized constructors for every distinct
CIT problem, which is not addressed by the existing constructors.

\section{EXPERIMENTS}

F-CIT does not aim to replace existing CIT constructors, but rather to reduce the barriers to applying CIT to other domains and problems. Note that, in this context, changing the underlying CIT problem is not the same as simply changing the parameters of an existing problem, but rather changing the problem itself. For example, for standard covering arrays, we don't consider the changes in system-wide constraints and/or the changes in model parameters to be a change in the underlying CIT problem. This is because the only thing that changes in such situations is the problem parameters, while the original problem remains intact, which is to cover all valid $t$-tuples at least once.

To evaluate F-CIT, we, therefore, carry out three case studies, each of which focuses on a different CIT problem. In the first study (Section 5.1), we compute structure-based CIT objects to obtain decision coverage-adequate objects. In the second study (Section 5.2), we compute order-based CIT objects, where the reachability constraints imposed by an underlying graph-based model are taken into account to cover various sequences of events. In the third study (Section 5.3), we compute usage-based CIT objects by selecting the tuples to be covered based on their usage statistics in the field, which is especially useful when standard covering arrays are not desirable due to their sizes.

In each study, we first introduce the CIT problem of interest and discuss the motivation behind this problem. We then discuss and empirically demonstrate that to compute the requested CIT objects, the existing constructors (as they are) require excessive number of test cases to guarantee full coverage. Or, they require non-trivial modifications. Or, it is not clear (if at all possible) how to modify them. We finally express the CIT problems in F-CIT and show that the very same FCIT constructor (thus, the same construction approach) can compute all of the requested CIT objects in all the studies without any modifications, demonstrating the flexibility of the proposed approach.

In the experiments, we integrate different "solvers" with F-CIT. This, however, is solely for the purpose of demonstrating that F-CIT can work with different solvers. The very same solver, such as the CSP solver we use in Section 5.1, can in deed be used in all the studies.

Note further that although the CIT problems in our studies are different than the ones addressed by existing CIT constructors, we opt to use existing constructors for comparisons in the experiments to justify the need for F-CIT. That is, in these studies, we are not claiming that F-CIT constructors perform better than 
standard CIT constructors (because the underlying CIT problems are different), but rather demonstrating that a different CIT constructor is indeed needed to compute the requested CIT objects in an efficient and effective manner. Otherwise, i.e., had the existing constructors addressed the CIT problems presented in this paper in an efficient and effective manner, there would be no need for F-CIT.

We, furthermore, use our generate-and-cover F-CIT constructor as a base line to show that computing FCIT objects is not trivial at all and that better construction approaches, such as the cover-and-generate approach, are needed.

The raw data we obtained from the experiments can be found at https://github.com/susoftgroup/UCIT/.

\subsection{Study 1: Structure-Based CIT}

In this study, we use the same CIT problem discussed in Section 2.

\subsubsection{Coverage criterion}

In [26], [12], we introduced a novel CIT object, which given a structural coverage criterion, such as decision coverage (DC), computes a "minimal" test suite to obtain full coverage under the criterion. In this work, we not only express the same coverage criterion using F-CIT, demonstrating the expressiveness of F-CIT, but also generalize the aforementioned coverage criterion to higher coverage strengths, demonstrating the flexibility of F-CIT. We call this structure-based CIT.

In a nutshell, structure-based CIT takes as input the source code of the system under test, a coverage strength $t$, and a structural code coverage criterion. First, for each outer-most if-then-else directive in the implementation, a virtual configuration option is defined. Then, for a given a virtual configuration option, conditions that must be satisfied to obtain a full coverage under the given structural coverage criterion for the respective if-then-else directive, are defined as virtual settings. Finally, a number of configurations are selected to cover all valid $t$-way combinations of virtual option settings. The smaller the number of configurations selected, the better the approach is.

Next, without losing generality, we provide more details by using DC as the structural code coverage criterion of interest. The proposed approach, on the other hand, is readily available to use with other structural coverage criteria, such as condition coverage [27].

Definition 11. A virtual configuration option (or virtual option, in short) represents an outer-most if-thenelse directive, which is not nested in another if-then-else directive.

For example, the system in Figure 1a has two virtual options: $v o_{1}$ representing the outer-most if-then-else directive between lines 1 and 5 and $v o_{2}$ representing the outer-most if-then-else directive between lines 6 and 10

Definition 12. Given a virtual configuration option, each feasible outcome of every decision in the respective if-thenelse directive, is defined as a virtual setting and expressed as a constraint, such that covering all of these virtual settings obtains a full coverage under DC.

For instance, the virtual option $v o_{1}$ in our running example has four virtual settings: $\left\{o_{1} \wedge o_{2}, \neg\left(o_{1} \wedge o_{2}\right)\right.$, $\left.\left(o_{1} \wedge o_{2}\right) \wedge\left(o_{3} \vee o_{4}\right),\left(o_{1} \wedge o_{2}\right) \wedge \neg\left(o_{3} \vee o_{4}\right)\right\}$. The first two settings are respectively for covering the true and false branches of the decision $o_{1} \wedge o_{2}$ and the last two settings are respectively for covering the true and false branches of the decision $o_{3} \vee o_{4}$ while taking the guard condition $o_{1} \wedge o_{2}$ into account. Similarly, $v o_{2}$ has four virtual settings: $\left\{o_{5}, \neg O_{5}, o_{5} \wedge o_{6}, o_{5} \wedge \neg o_{6}\right\}$.

Not all virtual settings of a virtual option may be valid due to some conflicting settings required for the actual configuration options that appear multiple times in the same if-then-else directive. Since each virtual setting is expressed as a constraint, an invalid virtual setting can be marked and filtered out by determining whether or not the respective constraint is satisfiable. That is, a virtual setting is invalid, if the respective constraint is not satisfiable. Clearly, covering invalid virtual settings is not required to achieve full coverage. Consequently, in the remainder of the paper, the term "virtual setting" is used to refer to valid virtual settings.

Definition 13. A t-combination is a combination of virtual settings for a combination of $t$ distinct virtual options, which is expressed by joining the respective constraints with the AND logical operator.

As was the case with virtual settings, a $t$ combination is invalid, if the respective constraint is not satisfiable. In the remainder of the paper, the term " $t$-combination" is used to refer to valid $t$ combinations.

Note that each $t$-combination represents an interaction that can be tested. Going back to our running example and considering that $t=2$, some example 2-combinations for the virtual options $v o_{1}$ and $v o_{2}$ are: $\left(o_{1} \wedge o_{2}\right) \wedge\left(o_{5}\right)$, testing the interaction between the true branches of the decisions at lines 1 and 6; and $\left(\left(o_{1} \wedge o_{2}\right) \wedge \neg\left(o_{3} \vee o_{4}\right)\right) \wedge\left(o_{6}\right)$, testing the interaction between the false branch of the decision at line 2 and the true branch of the decision at line 7 .

Definition 14. Given a set of virtual configuration options, their virtual settings, and a coverage strength $t, \mathrm{t}$ way structure-based coverage criterion $K_{\text {struct }}$ marks all valid t-combinations for coverage.

Definition 15. Given a set of virtual configuration options, their virtual settings, and a coverage strength $t, a$ t-way structure-based F-CIT object is a set of actual system 
TABLE 2: Information about the subject applications used in Study 1.

\begin{tabular}{|c|c|c|c|c|c|c|c|}
\hline sut & version & description & $\begin{array}{r}\text { actual } \\
\text { options }\end{array}$ & $\begin{array}{r}\text { virtual } \\
\text { options }\end{array}$ & $\begin{array}{r}\text { valid } \\
1 \text {-combins }\end{array}$ & $\begin{array}{r}\text { valid } \\
2 \text {-combins }\end{array}$ & $\begin{array}{r}\text { valid } \\
\text { 3-combins }\end{array}$ \\
\hline mpsolve & 2.2 & Mathematical solver & 14 & 4 & 30 & 296 & 1104 \\
\hline dia & 0.96 .1 & Diagramming application & 15 & 11 & 42 & 734 & 7170 \\
\hline irissi & 0.8 .13 & IRC client & 30 & 11 & 70 & 2102 & 36056 \\
\hline xterm & 2.4 .3 & Terminal emulator & 38 & 31 & 78 & 2871 & 66497 \\
\hline parrot & 0.9 .1 & Virtual machine & 51 & 29 & 152 & 10359 & 426194 \\
\hline $\operatorname{gimp}$ & 3.2.5 & Vector graphics editor & 79 & 28 & 198 & 16438 & 794050 \\
\hline pidgin & 2.4 .0 & IM & 53 & 43 & 199 & 17857 & 986926 \\
\hline python & 2.6 .4 & Programming language & 68 & 49 & 210 & 21180 & 1368012 \\
\hline xfig & 2.6 .8 & Graphics manipulator & 79 & 48 & 237 & 26985 & 1969006 \\
\hline vim & 7.3 & Text editor & 79 & 49 & 239 & 27442 & 2019176 \\
\hline sylpheed & 2.6.0 & E-mail client & 84 & 48 & 258 & 31597 & 2451586 \\
\hline cherokee & 1.0 .2 & Web server & 97 & 28 & 272 & 32530 & 2318986 \\
\hline
\end{tabular}

configurations, in which each t-combination selected by $K_{\text {struct }}$ is covered by at least one configuration.

In this context, an actual system configuration is said to cover a $t$-combination, if the configuration is consistent with the respective constraint.

Note further that the coverage strength $t$ in $K_{\text {struct }}$ can be 1 , which simply marks the virtual settings of all the virtual options for coverage. Therefore, covering all valid 1-combinations (i.e., all virtual settings) guarantees to obtain full coverage under DC. Consequently, 1way structure-based F-CIT objects are the same/similar combinatorial objects we introduced in our short paper [12], but expressed in F-CIT, demonstrating the expressiveness of F-CIT.

One issue with the 1-way structure-based F-CIT objects, however, is that they don't take the interactions between structurally isolated if-then-else directives into account. Take the 1-way structure-based object given in Figure $1 \mathrm{~d}$ as an example, although a DC-adequate test suite, it does not, for example, test the interaction between the true branch of the decision $o_{1} \wedge o_{2}$ (line 1) and the false branch of the decision $o_{5}$ (line 6).

This issue, which was not addressed in our previous work [12], can now easily be handled in F-CIT by simply increasing the strength of $K_{\text {struct }}$, demonstrating the flexibility of F-CIT by generalizing the coverage criterion introduced in [12]. Going back to our running example in Figure 1 and considering that $t=2, K_{\text {struct }}$ selects $4 * 4=162$-combinations for $v o_{1}$ and $v o_{2}$, covering all the pairwise interactions between the settings of these virtual options.

\subsubsection{Study setup}

For the evaluations, we used 12 subject applications. Each application had a number of binary compile-time configuration options implemented by using preprocessor directives. Table 2 provides information about these subject applications. The columns of this table
TABLE 3: Percentages of the if-then-else directives (one per virtual option) that are of cyclomatic complexity $2,3,4,5$, and $\geq 6$.

\begin{tabular}{|l|rrrrr|}
\hline & \multicolumn{5}{|c|}{ cyclomatic complexity } \\
sut & 2 & 3 & 4 & 5 & $\geq 6$ \\
\hline mpsolve & 0 & 50 & 0 & 0 & 50 \\
dia & 9.09 & 63.64 & 27.27 & 0 & 0 \\
irissi & 0 & 36.36 & 36.36 & 0 & 27.27 \\
xterm & 54.84 & 25.81 & 6.45 & 6.45 & 6.45 \\
parrot & 24.14 & 37.93 & 13.79 & 6.90 & 17.24 \\
gimp & 0 & 57.14 & 10.71 & 28.57 & 3.57 \\
pidgin & 2.33 & 53.49 & 25.58 & 9.30 & 9.30 \\
python & 8.16 & 63.27 & 16.33 & 4.08 & 8.16 \\
xfig & 2.08 & 50 & 20.83 & 14.58 & 12.50 \\
vim & 4.08 & 48.98 & 20.41 & 14.29 & 12.24 \\
sylpheed & 10.42 & 56.25 & 8.33 & 6.25 & 18.75 \\
cherokee & 3.57 & 32.14 & 14.29 & 7.14 & 42.86 \\
\hline
\end{tabular}

respectively present the subject applications, their versions and descriptions, the numbers of actual compiletime options they have, the numbers of virtual options extracted, and the numbers of 1-, 2- and 3combinations selected by our structure-based coverage criterion. Note that since we were not aware of any inter-option constraints for these subject applications, all possible combinations of option settings were considered to be valid. Furthermore, to give an idea about the structural complexities of the virtual options we extracted, Table 3 presents the percentages of the virtual options that are of cyclomatic complexities of 2 , $3,4,5$, and $\geq 6$, respectively. Throughout the paper cyclomatic complexities are computed on a per virtual option basis by using Radon [28] - a tool to compute various code metrics.

All the experiments, unless otherwise stated, were repeated 5 times and carried out on Google Cloud using Intel Xeon CPU 2.30GHz machine with 4 GB of 
RAM, running 64-bit Ubuntu 17.10 as the operating system.

\subsubsection{Applying standard CIT}

Modeling. The very first observation we make is that standard covering arrays cannot be used (as they are) with virtual options because the settings of virtual options are constraints, rather than discrete values as is the case with standard covering arrays. For example, one setting for $v o_{1}$ is $\left(o_{1} \wedge o_{2}\right) \wedge\left(o_{3} \vee o_{4}\right)$ and another is $\left(o_{1} \wedge o_{2}\right) \wedge \neg\left(o_{3} \vee o_{4}\right)$. To the best of our knowledge, there is no standard covering array constructor that can take constraints as settings. Note that these virtual settings cannot be expressed as constraints in standard constructors either, because such constraints are globally enforced and virtual settings can conflict with each other, which prevents the creation of any covering arrays (Section 2).

An alternative approach can be to create a standard covering array for the actual configuration options to obtain full coverage under $K_{\text {struct }}$. This, however, may unnecessarily increase the number of configurations required. For example, the standard 2-way covering array given in Figure $1 \mathrm{~b}$ obtains only $38 \%$ coverage under the 2-way $K_{\text {struct }}$ criterion (covering only 9 out of 242 -combinations). Since the maximum number of actual configuration options involved in a 2-combination is 6 in this example, a 6 -way covering array needs to be used to guarantee full coverage. This, however, is the same as exhaustive testing. Indeed, using variable strength covering arrays as an alternative, also suffers from the same issue.

Next, to demonstrate that the CIT problem defined in this study is indeed different than the ones addressed by standard covering arrays, which justifies the need for a different constructor to guarantee full coverage in an efficient and effective manner, we apply standard CIT on the subject applications in Table 2.

Evaluations. We first observed that since standard covering arrays do not necessarily take the complex interactions between configuration options into account, they, especially in the presence of tangled options, either fail to obtain full decision coverage or require excessive number of test cases [26], [12].

More specifically, we first created standard 2-way and 3-way covering arrays for our subject applications and measured the $t$-way structure-based coverage they provided for $t=1,2$, and 3 . The experiments for $t=1$ and 2 were repeated 30 times, whereas those for $t=3$ were repeated 5 times as measuring the coverage for higher strengths was costly. The average sizes of the standard 2-way and 3-way covering arrays created were 13.74 and 36.78 , respectively.

Standard covering arrays did not even guarantee DC adequacy, i.e., 1-way structure-based coverage (Table 4). More specifically, in about $58 \%$ (14 out of 24 )
TABLE 4: Percentages of the 1-, 2-, and 3 -combinations covered by standard 2- and 3-way covering arrays. The experiments were repeated 30 times.

\begin{tabular}{|l|rrr|rrr|}
\hline \multirow{3}{*}{ sut } & \multicolumn{3}{|c|}{ standard 2-way CA } & \multicolumn{3}{c|}{ standard 3-way CA } \\
\cline { 2 - 6 } & \multicolumn{3}{|c|}{$\begin{array}{c}\text { \% } \text {-combinations } \\
\text { covered }\end{array}$} & \multicolumn{3}{c|}{$\begin{array}{c}t \text {-combinations } \\
\text { covered }\end{array}$} \\
\cline { 2 - 7 } & $t=1$ & $t=2$ & $t=3$ & $t=1$ & $t=2$ & $t=3$ \\
\hline mpsolve & 100 & 55 & 23 & 100 & 83 & 56 \\
dia & 99 & 39 & 18 & 100 & 46 & 27 \\
irissi & 100 & 36 & 11 & 100 & 49 & 22 \\
xterm & 97 & 49 & 29 & 98 & 55 & 38 \\
parrot & 90 & 29 & 8 & 94 & 33 & 15 \\
gimp & 95 & 36 & 14 & 98 & 47 & 21 \\
pidgin & 99 & 23 & 11 & 100 & 25 & 17 \\
python & 98 & 31 & 12 & 99 & 36 & 18 \\
xfig & 99 & 31 & 12 & 100 & 35 & 18 \\
vim & 99 & 30 & 11 & 100 & 34 & 18 \\
sylpheed & 97 & 39 & 16 & 98 & 45 & 25 \\
cherokee & 99 & 21 & 5 & 100 & 28 & 10 \\
\hline
\end{tabular}

TABLE 5: Percentages of valid 1-combinations of various cyclomatic complexities covered by standard $t$-way covering arrays.

\begin{tabular}{|c|r|r|}
\hline \multirow{2}{*}{$\begin{array}{c}\text { cyclomatic } \\
\text { complexity }\end{array}$} & \multicolumn{2}{|c|}{$\begin{array}{c}\text { standard } t \text {-way } \\
\text { covering arrays }\end{array}$} \\
\cline { 2 - 3 } & $t=2$ & $t=3$ \\
\hline 2 & 100.00 & 100.00 \\
3 & 100.00 & 100.00 \\
4 & 98.96 & 100.00 \\
5 & 98.17 & 99.84 \\
$\geq 6$ & 94.17 & 97.28 \\
\hline
\end{tabular}

of the experimental setups, standard covering arrays could not obtain full DC coverage. Overall, the DC coverages achieved were $97.58 \%$ and $99.08 \%$, on average, for $t=2$ and 3 , respectively.

Furthermore, the higher the strength of the structurebased criterion, the more the required combinations were missing from the standard covering arrays (Table 4). Overall, the 2- and 3-way standard covering arrays, while respectively covering $34.92 \%$ and $43.00 \%$ of all the 2 -combinations, achieved $14.17 \%$ and $23.75 \%$ coverage of the 3-combinations.

Similarly, the more the cyclomatic complexity of the virtual options, the more the required combinations were missing (Table 5). For example, standard 2-way covering arrays, on average, covered $100.00 \%, 100.00 \%$, $98.96 \%, 98.17 \%$, and $94.17 \%$ of the 1 -combinations for the virtual options with cyclomatic complexities of 2 , $3,4,5$, and $\geq 6$, respectively.

We have then created higher strength as well as variable strength covering arrays. For the former, we determined the maximum number of distinct configuration options that appear in a $t$-way virtual option combination and used it as the strength of the standard covering array. For the latter, we determined the number of distinct configuration options that appear 
TABLE 6: Using standard covering arrays to guarantee full coverage under structure-based coverage criterion. The columns indicate the subject application, the coverage strength of the standard covering array computed together with the average construction time and size obtained by repeating the experiments 3 times for 1-, 2-, and 3-way structure-based CIT, respectively. The symbol '-' marks experimental setups, for which the standard constructor failed with an "out of memory" exception.

\begin{tabular}{|l|rrr|rrr|rrr|}
\hline \multirow{3}{*}{ sut } & \multicolumn{9}{|c|}{$t$-way standard covering arrays created for structure-based CIT } \\
\cline { 2 - 10 } & \multicolumn{3}{|c|}{$\begin{array}{l}\text { 1-way structure- } \\
\text { based CIT }\end{array}$} & \multicolumn{3}{c|}{$\begin{array}{c}\text { 2-way structure- } \\
\text { based CIT }\end{array}$} & \multicolumn{3}{c|}{$\begin{array}{c}\text { 3-way structure } \\
\text { based CIT }\end{array}$} \\
\cline { 2 - 10 } & $\mathrm{t}$ & time & size & t & time & size & t & time & size \\
\hline mpsolve & 2 & 0.34 & 10 & 4 & 0.33 & 54 & 6 & 0.56 & 272 \\
dia & 3 & 0.36 & 26 & 5 & 0.46 & 134 & 7 & 0.97 & 608 \\
irissi & 4 & 0.90 & 82 & 7 & - & - & 9 & - & - \\
xterm & 9 & - & - & 12 & - & - & 15 & - & - \\
parrot & 10 & - & - & 15 & - & - & 18 & - & - \\
xfig & 6 & - & - & 9 & - & - & 12 & - & - \\
python & 5 & 616.80 & 299 & 9 & - & - & 12 & - & - \\
pidgin & 8 & - & - & 11 & - & - & 14 & - & - \\
gimp & 5 & - & - & 10 & - & - & 15 & - & - \\
vim & 5 & - & - & 10 & - & - & 15 & - & - \\
sylpheed & 10 & - & - & 16 & - & - & 20 & - & - \\
cherokee & 4 & 73.77 & 130 & 7 & - & - & 10 & - & - \\
\hline
\end{tabular}

in each t-way virtual option combination and used it as the coverage strength to be satisfied for these configuration options. All of the covering arrays in these experiments were computed by using ACTS [29] and the experiments were repeated 3 times.

Tables 6-7 present the results we obtained. In $75 \%$ (27 out of 36) of the experimental setups for computing fixed-strength covering arrays and in $28 \%$ (10 out of 36) of the experimental setups for computing variable strength covering arrays, the standard constructor (ACTS) failed with an "out of memory" exception. The tables, therefore, present only the experiments, in which we were able to compute a covering array using the standard constructor. Although the covering arrays we could compute achieved full coverage, they did so at the expense of excessive number of configurations. For comparisons, the interesting reader can refer to Table 8 to check the sizes of the F-CIT objects computed for the study.

\subsubsection{Applying F-CIT}

Modeling. We have defined the F-CIT model as $M=\langle P, D, C\rangle$, where $P$ is the set of variables representing the actual configuration options; $D$ is their respective domains, i.e., the settings that the actual configuration options can take on; and $C$ is the model constraint (if any) invalidating certain combinations of option settings. Each F-CIT testable entity then naturally corresponded to a valid $t$-combination to be covered (Definition 13) and each F-CIT test case naturally corresponded to a configuration, in which every actual configuration option has a valid setting.

We have also used the seeding mechanism of F-CIT (Section 4.3) in this study to combine multiple coverage
TABLE 7: Using variable strength covering arrays to guarantee full coverage under structure-based coverage criterion. The columns indicate the subject application and the average construction time and size of the variable strength covering arrays computed for 1-, 2-, and 3-way structure-based CIT, respectively. The experiments were repeated 3 times. The symbol '-' marks experimental setups, for which the standard constructor failed with an "out of memory" exception.

\begin{tabular}{|l|rr|rr|rr|}
\hline \multirow{3}{*}{ sut } & \multicolumn{2}{|c|}{ variable strength covering arrays created for structure-based CIT } \\
\cline { 2 - 7 } & \multicolumn{2}{|c|}{$\begin{array}{l}\text { 1-way structure- } \\
\text { based CIT }\end{array}$} & \multicolumn{2}{c|}{$\begin{array}{c}\text { 2-way structure- } \\
\text { based CIT }\end{array}$} & \multicolumn{2}{|c|}{$\begin{array}{c}\text { 3-way structure- } \\
\text { based CIT }\end{array}$} \\
\cline { 2 - 7 } & time & size & time & size & time & size \\
\hline mpsolve & 0.29 & 8 & 0.41 & 47 & 0.88 & 252 \\
dia & 0.32 & 8 & 0.42 & 48 & 0.79 & 202 \\
irissi & 0.33 & 16 & 0.99 & 323 & 554.99 & 3217 \\
xterm & 0.54 & 512 & 12.05 & 4187 & - & - \\
parrot & 5.49 & 3750 & - & - & - & - \\
xfig & 364.78 & 585 & - & - & - & - \\
python & 0.40 & 32 & 4.70 & 845 & 6319.56 & 13350 \\
pidgin & 0.44 & 256 & 15.90 & 3447 & - & - \\
gimp & 0.41 & 32 & 6.07 & 730 & 4317.48 & 8908 \\
vim & 0.41 & 36 & 4.82 & 718 & 43198.74 & 9037 \\
sylpheed & 19.62 & 5062 & - & - & - & - \\
cherokee & 0.43 & 18 & - & - & - & - \\
\hline
\end{tabular}

criteria. In particular, to construct 1-way structurebased F-CIT objects in some experiments, we used standard 2-way or 3-way covering arrays computed for the actual configuration options, as seeds. By doing so, we effectively computed $t$-way DC-adequate covering arrays, which not only covered all $t$-way combinations of actual option settings, but also achieved DC adequacy.

To further demonstrate that the very same seeding mechanism can also be used to incrementally compute F-CIT objects - a well-known approach for computing standard covering arrays [30], we have used lower strength structure-based F-CIT objects as seeds to compute higher strength F-CIT objects.

Cost. To extract virtual options from source code, we used cppstats, which is a static analysis tool for analyzing $\mathrm{C} / \mathrm{C}++$ preprocessor-based variability in highly configurable systems [31]. The tool parsed the if-thenelse directives into an XML-based tree representation. We then simply traversed the representation to identify the elements that corresponded to virtual options. An if-then-else directive, which was not structurally contained in another if-then-else directive simply became a virtual option. Once a virtual option was found, we traversed the respective tree to determine the virtual settings, i.e., visiting the decisions in the possibly nested if-then-else directive. For each decision $d$ with a guard condition $g$, two virtual settings were created: $g \wedge d$ and $g \wedge \neg d$. All told, developing a generic script to carry out these steps took about 10 hours.

We have integrated our constructors given in Algorithms 1 and 2 with SATisPy [32], which is a Python library that interfaces with various SAT solvers, such as MiniSat [33]. Since the decisions in the source code 
TABLE 8: Information about the structure-based F-CIT objects created. The symbol '*' marks the experimental setups, in which the generate-and-cover constructor timed out after six days. The experiments were repeated 5

times.

\begin{tabular}{|l|rr|rr|rr|rr|rrr|r|r|r|}
\hline & \multicolumn{4}{|c|}{ 1-way } & \multicolumn{4}{c|}{ 2-way } & \multicolumn{4}{c|}{ 3-way } \\
\hline & \multicolumn{2}{|c|}{$\begin{array}{c}\text { generate- } \\
\text { and-cover }\end{array}$} & \multicolumn{2}{c|}{$\begin{array}{c}\text { cover-and- } \\
\text { generate }\end{array}$} & \multicolumn{2}{c|}{$\begin{array}{c}\text { generate- } \\
\text { and-cover }\end{array}$} & \multicolumn{2}{c|}{$\begin{array}{c}\text { cover-and- } \\
\text { generate }\end{array}$} & \multicolumn{2}{c|}{$\begin{array}{c}\text { generate- } \\
\text { and-cover }\end{array}$} & \multicolumn{2}{c|}{$\begin{array}{c}\text { cover-and- } \\
\text { generate }\end{array}$} \\
\hline sut & time & size & time & size & time & size & time & size & time & size & time & size \\
\hline mpsolve & 0.37 & 3.00 & 0.31 & 3.00 & 17.61 & 15.20 & 2.07 & 14.00 & 221.54 & 93.40 & 11.99 & 39.80 \\
dia & 0.37 & 4.40 & 0.34 & 4.20 & 16.35 & 19.60 & 2.26 & 19.40 & 482.35 & 131.80 & 24.79 & 70.60 \\
irissi & 0.69 & 4.00 & 0.66 & 4.00 & 74.21 & 25.20 & 13.16 & 24.20 & 8461.64 & 316.40 & 139.32 & 109.20 \\
xterm & 0.61 & 4.20 & 0.58 & 4.20 & 50.54 & 19.80 & 5.74 & 21.20 & 7025.89 & 271.60 & 92.54 & 79.00 \\
parrot & 2.03 & 10.00 & 1.95 & 10.00 & 877.18 & 57.80 & 46.65 & 55.80 & 206682.44 & 841.33 & 1070.67 & 317.40 \\
gimp & 2.45 & 8.20 & 2.27 & 8.00 & 825.78 & 49.80 & 67.11 & 48.00 & 457184.81 & 998.50 & 1645.61 & 272.80 \\
pidgin & 2.26 & 4.40 & 2.29 & 4.40 & 788.98 & 34.00 & 31.82 & 33.40 & & $*$ & $*$ & 628.75 & 172.00 \\
python & 2.16 & 4.80 & 2.07 & 4.40 & 743.89 & 36.00 & 28.68 & 34.60 & & $*$ & $*$ & 932.46 & 187.00 \\
xfig & 2.81 & 5.80 & 2.74 & 6.00 & 1355.77 & 46.00 & 78.54 & 45.80 & & $*$ & $*$ & 2311.84 & 270.00 \\
vim & 2.82 & 6.40 & 2.69 & 6.20 & 1357.64 & 48.60 & 56.47 & 48.60 & & $*$ & $*$ & 1679.70 & 291.20 \\
sylpheed & 3.18 & 6.00 & 3.04 & 6.60 & 1737.00 & 49.20 & 78.20 & 47.40 & & $*$ & $*$ & 2724.60 & 279.20 \\
cherokee & 3.59 & 5.00 & 3.53 & 5.00 & 2792.24 & 45.40 & 79.89 & 45.00 & & $*$ & $*$ & 2095.94 & 252.40 \\
\hline
\end{tabular}

were already expressed as Boolean expressions and since the virtual settings (thus, the testable entities) were simply obtained by joining these expressions (or their negations) with the AND logical operator, the integration step took about 1 hour. Most of this time was, indeed, spent for developing simple syntactic transformations to match the input format of the solver. Furthermore, since all the testable entities in this study are expressed in Boolean logic, the SATisPy solver, which we opted to use in the first place due to its easeof-use, can easily be replaced with any other SAT or CSP solver.

Evaluations. The $t$-way structure-based F-CIT objects we computed in this study covered all the required $t$-combinations by construction. Furthermore, the cover-and-generate constructor generally performed better than the generate-and-cover constructor in reducing both the sizes and the construction times (Table 8). We, therefore, ran the generate-and-cover constructor with a time-out period of six days per construction. Overall, the cover-and-generate constructor reduced the sizes by an average of $2 \%, 77 \%$, and $66 \%$, while at the same time reducing the construction times by an average of $3.31 \%, 95.39 \%$, and $99.56 \%$, when $t=1,2$, and 3, respectively. Note further that in $16.67 \%$ (6 out of 36 ) of the experimental setups, te generate-and-cover constructor timed out (Table 8). $\mathrm{We}$, therefore, focus on the results obtained from the cover-and-generate constructor in the remainder of this section.

As expected, the higher the coverage strength, the larger the size and the construction time of the structure-based F-CIT objects tended to be. More specifically, the average sizes were $5.50,36.45$, and 195.05 with the average constructions times of 1.87 , 40.88 , and 1113.18 seconds for 1-, 2-, and 3-way structure-based F-CIT objects, respectively.
TABLE 9: Information about the $t$-way DC-adequate covering arrays created by computing 1-way structure-based F-CIT objects using $t$-way standard covering arrays as seeds. The column '+cfgs.' reports the average numbers of additional configurations needed. The experiments were repeated 5 times.

\begin{tabular}{|l|cc|cc|cc|cc|}
\hline \multirow{3}{*}{ sut } & \multicolumn{3}{|c|}{$\begin{array}{c}\text { using 2-way standard } \\
\text { CAs as seeds }\end{array}$} & \multicolumn{3}{c|}{$\begin{array}{c}\text { using 3-way standard } \\
\text { CAs as seeds }\end{array}$} \\
\cline { 2 - 9 } & $\begin{array}{c}\text { generate- } \\
\text { and-cover } \\
\text { constructor }\end{array}$ & \multicolumn{2}{c|}{$\begin{array}{c}\text { cover-and- } \\
\text { generate } \\
\text { constructor }\end{array}$} & $\begin{array}{c}\text { generate- } \\
\text { and-cover } \\
\text { constructor }\end{array}$ & $\begin{array}{c}\text { cover-and- } \\
\text { generate } \\
\text { constructor }\end{array}$ \\
\cline { 2 - 9 } mpsolve & 0.72 & 0.00 & 0.61 & 0.00 & 0.70 & 0.00 & 0.60 & 0.00 \\
dia & 0.47 & 0.00 & 0.42 & 0.00 & 0.45 & 0.00 & 0.40 & 0.00 \\
irissi & 1.07 & 1.00 & 0.83 & 1.00 & 1.05 & 0.00 & 0.83 & 0.00 \\
xterm & 0.74 & 3.80 & 0.86 & 1.00 & 0.77 & 0.00 & 0.92 & 0.00 \\
parrot & 3.84 & 12.40 & 3.53 & 7.00 & 4.25 & 6.00 & 4.14 & 5.00 \\
gimp & 5.68 & 12.20 & 3.98 & 3.00 & 6.28 & 4.60 & 4.63 & 2.00 \\
pidgin & 2.53 & 1.00 & 3.09 & 1.00 & 2.71 & 0.00 & 3.31 & 0.00 \\
python & 3.82 & 5.00 & 3.51 & 2.00 & 3.85 & 0.00 & 3.61 & 0.00 \\
xfig & 4.23 & 3.00 & 4.24 & 1.00 & 4.41 & 0.00 & 4.20 & 0.00 \\
vim & 3.72 & 3.40 & 4.12 & 3.00 & 3.64 & 0.00 & 4.14 & 0.00 \\
sylpheed & 5.08 & 3.40 & 4.57 & 2.00 & 5.71 & 1.00 & 5.02 & 1.00 \\
cherokee & 5.80 & 3.00 & 6.12 & 1.00 & 6.22 & 1.00 & 6.13 & 1.00 \\
\hline
\end{tabular}

Computing t-way DC-adequate covering arrays. Note that as the ultimate goal of the structure-based F-CIT objects is to obtain full coverage under the $K_{\text {struct }}$ coverage criterion, they may not cover all the standard $t$-tuples. For example, the 1-way structure-based FCIT objects we generated covered $67.33 \%$ and $40.00 \%$ of all the 2- and 3-tuples, on average, respectively. The numbers were $94.33 \%$ and $86.33 \%$ for the 2-way structure-based and $95.17 \%$ and $91.75 \%$ for the 3-way structure-based F-CIT objects.

One good thing about having a seeding mechanism in F-CIT is that it can be leveraged to satisfy multiple coverage criteria. For example, one way to obtain $t$ way DC-adequate covering arrays, i.e., standard $t$-way covering arrays that guarantee full DC coverage, is 
TABLE 10: Using structure-based F-CIT objects as seeds to cover the missing 2- and 3-tuples by computing standard covering arrays. The column '+cfgs.' reports the average numbers of additional configurations needed. The experiments were repeated 5 times.

\begin{tabular}{|c|c|c|c|c|c|c|c|c|c|c|c|c|}
\hline \multirow{4}{*}{ sut } & \multicolumn{6}{|c|}{ standard 2-way CA } & \multicolumn{6}{|c|}{ standard 3-way CA } \\
\hline & \multicolumn{6}{|c|}{ using $t$-way structure-based objects as seeds } & \multicolumn{6}{|c|}{ using $t$-way structure-based objects as seeds } \\
\hline & \multicolumn{2}{|c|}{$t=1$} & \multicolumn{2}{|c|}{$t=2$} & \multicolumn{2}{|c|}{$t=3$} & \multicolumn{2}{|c|}{$t=1$} & \multicolumn{2}{|c|}{$t=2$} & \multicolumn{2}{|c|}{$t=3$} \\
\hline & time & + cfgs. & time & +cfgs. & time & +cfgs. & time & + cfgs. & time & + +cfgs. & time & + +cfgs. \\
\hline mpsolve & 0.07 & 7.00 & 0.06 & 2.40 & 0.06 & 0.80 & 0.07 & 19.40 & 0.08 & 13.20 & 0.07 & 8.40 \\
\hline dia & 0.06 & 6.80 & 0.07 & 2.00 & 0.07 & 2.00 & 0.08 & 18.80 & 0.08 & 11.60 & 0.08 & 9.20 \\
\hline irissi & 0.07 & 8.80 & 0.08 & 2.00 & 0.10 & 2.00 & 0.16 & 27.80 & 0.16 & 16.60 & 0.23 & 34.80 \\
\hline xterm & 0.08 & 9.00 & 0.09 & 7.00 & 0.13 & 7.00 & 0.24 & 31.20 & 0.24 & 25.20 & 0.31 & 24.40 \\
\hline parrot & 0.10 & 8.40 & 0.14 & 4.40 & 0.24 & 4.00 & 0.41 & 34.40 & 0.48 & 21.20 & 0.66 & 18.00 \\
\hline gimp & 0.15 & 10.80 & 0.22 & 8.20 & 0.35 & 7.80 & 0.97 & 39.80 & 1.19 & 30.40 & 1.47 & 28.20 \\
\hline pidgin & 0.10 & 10.00 & 0.12 & 7.00 & 0.21 & 7.00 & 0.42 & 35.40 & 0.46 & 24.60 & 0.69 & 29.80 \\
\hline python & 0.13 & 10.40 & 0.19 & 5.00 & 0.27 & 5.00 & 0.72 & 38.60 & 0.70 & 24.20 & 1.19 & 58.60 \\
\hline xfig & 0.16 & 10.80 & 0.22 & 3.00 & 0.34 & 3.00 & 1.04 & 40.00 & 1.05 & 21.00 & 1.43 & 23.60 \\
\hline vim & 0.15 & 11.00 & 0.20 & 3.00 & 0.34 & 3.00 & 0.97 & 40.20 & 1.10 & 20.80 & 1.40 & 24.00 \\
\hline sylpheed & 0.16 & 10.60 & 0.22 & 4.80 & 0.39 & 4.80 & 1.25 & 41.00 & 1.48 & 24.00 & 1.65 & 26.00 \\
\hline cherokee & 0.19 & 12.00 & 0.27 & 7.40 & 0.48 & 6.80 & 1.73 & 43.80 & 2.24 & 29.80 & 2.26 & 26.00 \\
\hline
\end{tabular}

TABLE 11: Information about the 3-way structure-based F-CIT objects created by using 2-way structure-based F-CIT objects as seeds. The experiments were repeated 5 times.

\begin{tabular}{|l|rc|rr|}
\hline \multirow{2}{*}{ sut } & \multicolumn{2}{|c|}{$\begin{array}{c}\text { generate-and-cover } \\
\text { constructor }\end{array}$} & \multicolumn{2}{c|}{$\begin{array}{c}\text { cover-and-generate } \\
\text { constructor }\end{array}$} \\
& \multicolumn{1}{c}{ time } & size & \multicolumn{1}{c|}{ time } & \multicolumn{1}{c|}{ size } \\
\hline mpsolve & 45.75 & 30.00 & 49.78 & 34.00 \\
dia & 152.62 & 58.20 & 65.38 & 59.60 \\
irissi & 2050.65 & 97.60 & 1029.66 & 92.00 \\
xterm & 594.90 & 73.20 & 167.30 & 71.60 \\
parrot & 18647.29 & 278.40 & 4103.58 & 279.60 \\
gimp & 12679.40 & 216.40 & 5563.53 & 215.80 \\
pidgin & 52514.79 & 158.80 & 30171.88 & 157.20 \\
python & 38510.67 & 170.40 & 16897.34 & 168.20 \\
xfig & 59537.75 & 230.40 & 14543.20 & 222.40 \\
vim & 67225.58 & 258.20 & 19227.10 & 247.80 \\
sylpheed & 117420.77 & 236.00 & 67550.43 & 243.80 \\
cherokee & 161779.40 & 211.20 & 57712.16 & 208.40 \\
\hline
\end{tabular}

to use standard $t$-way covering arrays as seeds to compute 1-way structure-based F-CIT objects.

To demonstrate the feasibility of this approach, we generated 2- and 3-way DC-adequate covering arrays (Table 9). We observed that 1-way structure-based FCIT objects turned the standard covering arrays into DC-adequate test suites with little increases in both the sizes and the construction times. The average numbers of additional configurations required on top of the standard 2-way and 3-way covering arrays were 1.83 and 0.75 , respectively, with the additional construction times of 2.99 and 3.16 seconds, on average.

Note that using structure-based F-CIT objects as seeds to compute standard covering arrays is also possible. To demonstrate the feasibility, we used, 1-, 2-, and 3-way structure-based F-CIT objects as seeds to compute 2- and 3-way standard covering arrays
TABLE 12: Information about the 4-way structure-based F-CIT objects created. Due to the cost, the experiments were repeated only once.

\begin{tabular}{|l|r|r|r|}
\hline sut & $\begin{array}{r}\text { valid } \\
\text { 4-combins }\end{array}$ & time & size \\
\hline mpsolve & 1344 & 16.24 & 62 \\
dia & 32346 & 111.32 & 197 \\
xterm & 615994 & 576.92 & 281 \\
irissi & 395504 & 2067.48 & 442 \\
pidgin & 15293336 & 16772.38 & 751 \\
python & 19856465 & 16958.23 & 869 \\
gimp & 14678226 & 42706.50 & 1293 \\
parrot & 7587625 & 19631.76 & 1482 \\
cherokee & 47087747 & 90360.34 & 2300 \\
sylpheed & 81732014 & 111090.57 & 3040 \\
xfig & 76405845 & 149335.77 & 3987 \\
vim & 76661558 & 96900.90 & 4340 \\
\hline
\end{tabular}

(Table 10). The average numbers of additional configurations required on top of the 1-, 2-, and 3-way structure-based F-CIT objects were 21.92, 13.28, and 15.18 , respectively, with the additional construction times of $0.40,0.46$, and 0.60 seconds, on average.

Incrementally computing structure-based F-CIT objects. Another use of the seeding mechanism is to leverage lower strength F-CIT objects as seeds for computing higher strength F-CIT objects. To demonstrate the feasibility, we used 2-way structure-based F-CIT objects as seeds to compute 3-way structure-based F-CIT objects. The results of these experiments can be found in Table 11.

Computing 4-way structure-based F-CIT objects. Last but not least, we have run our cover-and-generate constructor for $t=4$. Table 12 presents the results we obtained. Overall, the minimum, the average, and the maximum sizes of the 4-way structure-based F-CIT objects we computed were 62,1587 , and 4340 with 
the construction times of $16.24,45544,03$, and 96900.90 seconds respectively.

\subsubsection{Discussion}

Standard covering arrays and structure-based F-CIT objects clearly employ different coverage criteria. We, therefore, do not claim that the F-CIT constructors developed in this work performed better than the standard CIT constructor used in the study. We rather demonstrate that a different CIT constructor is indeed needed to obtain full coverage under the structurebased coverage criterion in an efficient and effective manner. Had the existing constructors addressed the structure-based CIT problem in an efficient and effective manner, there would be no need for F-CIT.

\subsection{Study 2: Order-Based CIT}

In this study, we use graphs to model the input spaces of software systems, which we believe can address many interesting test scenarios, such as the ones that arise during the systematic testing of event-driven systems as well as multi-threaded applications. We first define the model of the input space in an abstract manner and briefly discuss two scenarios in which the same or similar models have been used for testing, then present a number of coverage criteria for which CIT can be used to satisfy and discuss the shortcomings of the state-of-the-art CIT approaches, and finally present how F-CIT overcomes these shortcomings.

The model of interest in this study, in its simplest form, is a directed graph $G=\left(V, E, v_{0}, v_{\perp}\right)$, where $V$ is a set of nodes; $E$ is a set of ordered pairs of the form $(v, w)$, representing a directed edge from node $v \in V$ to node $w \in V$; and $v_{0} \in V$ and $v_{\perp} \in V$ are two distinguished nodes, namely the entry and the exit node. The entry node has an in-degree of 0 and the exit node has an out-degree of 0 . Furthermore, all the nodes are reachable from the entry node and the exit node is reachable from all the nodes. Figure 2 presents some example models.

Given a graph-based model, one high-level testing objective is to generate test cases to satisfy some structural coverage criterion, such as exercising every node and/or edge at least once [34]. When graphs are used as a model, however, the coverage criterion is often concerned with the order of the entities (e.g., nodes and edges) to be tested. For Figure 2a, one such criterion for example, would be to generate a set of paths from the entry node to the exit node, such that every valid order of two (not necessarily distinct) nodes is covered (not necessarily in a consecutive manner) by at least one path. Given this criterion, some example orders to be covered for Figure 2a are: $\left[v_{3}, v_{4}\right],\left[v_{1}, v_{6}\right],\left[v_{6}, v_{6}\right]$, and $\left[v_{6}, v_{5}\right]$, which can all be covered (together with other orders) by the path $\left(v_{0}, v_{1}, v_{3}, v_{4}, v_{5}, v_{6}, v_{5}, v_{6}, v_{\perp}\right)$. On

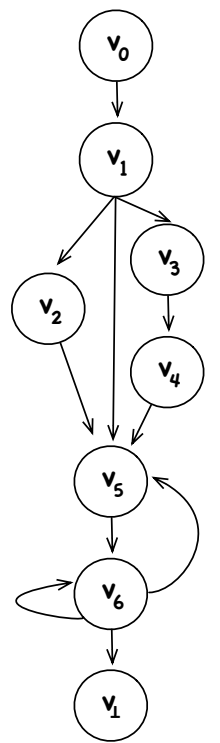

(a)

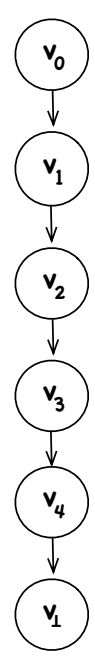

(b)

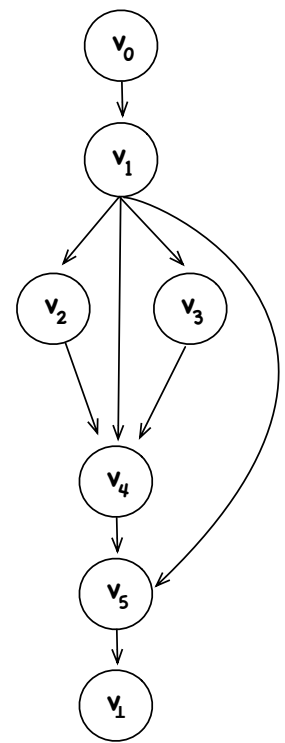

(c)
Fig. 2: Example graph-based models.

the other hand, $\left[v_{2}, v_{3}\right]$ and $\left[v_{2}, v_{4}\right]$ are not valid orders since no paths can include them.

The same and similar graph-based models and coverage criteria have indeed been used for software testing. For example, in systematic testing of event-driven systems, such as graphical user interfaces (GUIs), graph-based models can capture the flow of events in the form of event sequence graphs [35] or event-flow graphs [36], [35], where each node represents an event and a directed edge from $v_{1}$ to $v_{2}$ indicates that event $v_{2}$ can follow event $v_{1}$. In this context, an event is considered as an environmental or a user stimulus that from the perspective of testing can be mimicked by a test case. Since the behavior of an event-driven system often depends on the order, in which the events are processed, testing such a system typically involves validating the system response under different event orders.

Another domain, in which graph-based models have been used for testing, concerns the systematic testing of multi-threaded applications. In this domain, the model of a thread captures all sequences of "atomic blocks" that might be traversed through the thread during its execution [37], [38]. In the remainder of the paper, the aforementioned models are referred to as atomic block flow graphs (AFGs).

Each node in an AFG represents an atomic block and the edges connecting the nodes represent the possible execution sequences of atomic blocks. For the programs adhering to a strict mutual-exclusion locking principle [38], an atomic block is defined as a code segment from one lock exit to the subsequent lock exit. And, a lock exit in this context corresponds to the release of a lock previously acquired 
for a synchronized code segment [38], [39], [40]. For such programs, testing approaches aim to reveal nondeadlock errors, namely atomicity-violation and orderviolation errors. Atomicity-violation errors occur when a sequence of operations that need to be carried in an atomic manner is erroneously divided into multiple atomic blocks, such that the atomicity of the entire operation cannot be guaranteed. Order-violation errors occur when an implicit execution order between two groups of atomic blocks is assumed, but not enforced, e.g., thread $A$ is assumed to start before thread $B$. To detect these errors, different orders of atomic blocks need to tested.

Note that for these and similar scenarios, since a test case (e.g., a path from the entry node to the exit node) can cover more than one order, the number of test cases to obtain full coverage under a given coverage criterion can be reduced by carefully constructing the test cases. Consequently, CIT approaches can be of practical help.

With standard covering arrays, however, the order of parameter values in a test case is assumed to have no effect on the fault revealing ability of the test case. For example, given a software configuration, such as the ones studied in Section 5.1, any permutation of the option settings constituting the configuration, covers exactly the same set of option setting combinations, thus all these permutations should detect the same faulty interactions. For the scenarios we are interested in this study, however, the order matters. Consequently, the types of CIT objects we need in this study are quite different than the one we have computed in Section 5.1.

\subsubsection{Coverage criterion}

To take the orders into account, a different type of covering array, called a sequence-covering array, was defined in [41] and a number of interesting orderbased coverage criteria were presented in [9]. In this study, we improve on these works by making both the coverage criteria and the construction approach take the reachability constraints imposed by a given graphbased model into account. Further discussion on this can be found in Section 5.2.3.

Definition 16. Given $G=\left(V, E, v_{0}, v_{\perp}\right)$, a path is an ordered sequence of nodes $\left(v_{i_{1}}, \ldots, v_{i_{n}}\right)$, such that $\left(v_{i_{j}}, v_{i_{j+1}}\right) \in E$ for $1 \leq j<n$.

Definition 17. Given $G=\left(V, E, v_{0}, v_{\perp}\right), a$ test case is $a$ path from $v_{0}$ to $v_{\perp}$.

For a given test case $p$ of length $n$, let $p_{i}$, where $0 \leq$ $i \leq n$, be the node located at position $i$ in the test case, such that $p_{0}=v_{0}$ and $p_{n}=v_{\perp}$.

Definition 18. Given $G=\left(V, E, v_{0}, v_{\perp}\right), \quad a \quad t$-order $\left[v_{i_{1}}, \ldots, v_{i_{t}}\right]$, where $v_{i_{j}} \in V$ for $1 \leq j \leq t$, is an ordered tuple of not-necessarily-distinct $t$ nodes, such that there exists a test case $p$, in which the nodes $\left[v_{i_{1}}, \ldots, v_{i_{t}}\right]$ appear in the order they are given (not necessarily in a consecutive manner, though). A test case $p$ of length $n$, such that $p_{m_{j}}=v_{i_{j}}$ and $0<m_{1}<m_{2}<\cdots<m_{t}<n$ for $1 \leq j \leq t$, is said to cover the $t$-order $\left[v_{i_{1}}, \ldots, v_{i_{t}}\right]$.

For instance, for the graph given in Figure $2 \mathrm{a},\left[v_{1}, v_{2}\right]$ and $\left[v_{1}, v_{6}\right]$, which are both covered by the test case $\left(v_{0}, v_{1}, v_{2}, v_{5}, v_{6}, v_{\perp}\right)$, are examples of 2 -orders, whereas $\left[v_{2}, v_{3}\right]$ is not a 2 -order, since there is no path from $v_{2}$ to $v_{3}$.

Definition 19. Given $G=\left(V, E, v_{0}, v_{\perp}\right)$, a consecutive-torder $\left[v_{i_{1}}, \ldots, v_{i_{t}}\right]$ is a t-order, such that there exists a test case $p$ of length $n$, where $v_{i_{j}} \in V$ and $p_{m_{j}}=v_{i_{j}}$ for $1 \leq$ $j \leq t$, and $m_{k+1}=m_{k}+1$ for $1 \leq k<t$, i.e., $\left[v_{i_{1}}, \ldots, v_{i_{t}}\right]$ is a subpath in path $p$. Such a test case $p$ is said to cover the consecutive-t-order $\left[v_{i_{1}}, \ldots, v_{i_{t}}\right]$.

For instance, for the graph given in Figure 2a, $\left[v_{1}, v_{2}\right],\left[v_{6}, v_{5}\right]$, and $\left[v_{6}, v_{6}\right]$, which all appear as subpaths in $\left(v_{0}, v_{1}, v_{2}, v_{5}, v_{6}, v_{5}, v_{6}, v_{6}, v_{\perp}\right)$, are examples of consecutive-2-orders, whereas $\left[v_{1}, v_{6}\right]$, although a 2order, is not a consecutive-2-order, as there is no edge from $v_{1}$ to $v_{6}$.

Definition 20. Given $G=\left(V, E, v_{0}, v_{\perp}\right), \quad$ a nonconsecutive-t-order $\left[v_{i_{1}}, \ldots, v_{i_{t}}\right]$ is a t-order, such that there exists a test case $p$ of length $n$, where $v_{i_{j}} \in V$ and $p_{m_{j}}=v_{i_{j}}$ for $1 \leq j \leq t$, and $m_{k+1}-m_{k}>1$ for at least one $1 \leq k<t$. Such a test case $p$ is said to cover the non-consecutive-t-order $\left[v_{i_{1}}, \ldots, v_{i_{t}}\right]$.

For Figure 2a, $\left[v_{1}, v_{5}\right]$ is an example of a nonconsecutive-2-order, because there is at least one path, e.g., $\left(v_{0}, v_{1}, v_{2}, v_{5}, v_{6}, v_{\perp}\right)$, where the nodes constituting the order can appear in a non-consecutive manner. On the other hand, $\left[v_{3}, v_{4}\right]$, although a 2-order, is not a nonconsecutive-2-order, because all the paths including this order have it in a consecutive manner.

Based on these definitions, we define the four coverage criteria given below (inspired from [9]). We call this order-based CIT.

Definition 21. Given $G=\left(V, E, v_{0}, v_{\perp}\right)$, a set of test cases $T$ is t-order adequate, if and only if for every t-order in $G$, there exists at least one test case in $T$, which covers it.

Definition 22. Given $G=\left(V, E, v_{0}, v_{\perp}\right)$, a set of test cases $T$ is t-cover adequate, if and only if for every consecutive$t$-order in $G$, there exists at least one test case in $T$, which covers it.

Definition 23. Given $G=\left(V, E, v_{0}, v_{\perp}\right)$, a set of test cases $T$ is $t^{+}$-cover adequate, if and only if for every nonconsecutive-t-order in $G$, there is at least one test case in $T$, which covers it.

Definition 24. Given $G=\left(V, E, v_{0}, v_{\perp}\right)$, a set of test cases $T$ is $t^{*}$-cover adequate, if and only if $T$ is both $t$-cover adequate and $t^{+}$-cover adequate. 
Note that to satisfy the $t$-order adequacy criterion, all possible $t$-orders need to be covered at least once regardless of whether they are covered in the form of a consecutive- or non-consecutive-t-order, whereas to satisfy the $t$-cover adequacy criterion all possible $t$-orders that can be covered in a consecutive manner need to be covered in the form of a consecutive-t-order. Similarly, to satisfy the $t^{+}$-cover adequacy criterion, all possible $t$-orders that can be covered in a nonconsecutive manner need to be covered in the form of a non-consecutive- $t$-order. Finally, $t^{*}$-cover adequacy criterion is different than the $t$-order adequacy criterion, because when a $t$-order can be covered both in a consecutive and non-consecutive manner, the $t^{*}$-cover adequacy criterion guarantees that it is covered in the form of both consecutive- and non-consecutive-t-order, whereas for the $t$-order adequacy criterion covering it in either way is enough.

\subsubsection{Study setup}

In this study, we used 171 AFGs (atomic block flow graphs) obtained from Apache ActiveMQ v5.9.1 [42] - a high-performance, open source message oriented middleware - to evaluate the proposed approach. We unrolled the cycles in these graphs once to get acyclic graphs, which is a frequently used approach in bounded model checking [43] (see Section 5.2.4 for more details). After being unrolled, these graphs had an average of 312.82 nodes ( $\min =12$ and $\max =3604)$ and an average of 493.23 edges $(\min =12$ and $\max =6566$ ). All the experiments were carried out on the same Google Cloud platform we used in Study 1 (Section 5.1).

Note that due to the volume of the data to be reported in this section, using tabular notations was simply out of the question. Therefore, we opted to present different views of the data as we see fit by using plots, such as Figure 3, or by using summary tables, such as Tables 13-14. The raw data can, however, be found at https://github.com/susoftgroup/UCIT/.

In the summary tables, we first divide the experiments into 4 almost equal-size partitions with increasing complexity either by using the number of settings each configuration option has (e.g., Table 15) or by using the number of F-CIT entities to be covered (e.g., Tables 13 and 14). For each partition, we then report the minimum, median, and maximum results obtained in the partition. For a better interpretation of the results, we also filter out the experimental setups, in which the number of testable entities to be covered is less than 10. Furthermore, the partitions are indicated in the summary tables by the unique ID numbers reported under the "part." column.

\subsubsection{Applying standard CIT}

Modeling. The coverage criteria we have defined in Section 5.2.1 are inspired from [9], which empirically demonstrates that these order-based criteria are effective in detecting faults in event-driven software systems, such as graphical user interfaces (GUIs).

On the other hand, although the aforementioned work presents an approach to generate order-based CIT objects for a given graph-based model, it does not provide a systematic way of taking the reachability constraints imposed by the underlying graph into account during the construction of these objects. Such constraints are rather attempted to be handled after a CIT object is constructed with the aim of converting the invalid test cases, which are erroneously selected due to the overlooked-for constraints, to valid ones. However, no systematic way of carrying this post-mortem analysis is provided in [9]. Therefore, this approach can generate many invalid test cases, which may not be trivially "fixed." For example, for the model given in Figure $2 b$, out of 24 possible permutations of 4 nodes (excluding $v_{0}$ and $v_{\perp}$ ), only one of them $(4.2 \%)$ is a valid test case, which is difficult to generate by chance. Invalid test cases is an important issue in CIT, because they often result in wasted testing resources [14], [15].

More specifically, the proposed construction approach in [9] uses standard covering arrays to compute order-based CIT objects. It takes as input a set of $e$ events, a coverage strength $t$, and a predetermined length $l$ for the test cases to be generated (i.e., only fixed-length test cases can be generated) and as output computes a standard $t$-way covering array for $l$ options, each of which can take on $e$ settings (one distinct setting per event). For example, to compute a 2-cover-adequate CIT object for the model given in Figure $2 \mathrm{a}$, the aforementioned approach would generate a standard 2-way covering array for 6 options (because the minimum length of a test case to guarantee the coverage of all consecutive-2-orders is 6 ), each of which has 6 settings (because the number of nodes except for $v_{0}$ and $v_{\perp}$ is 6 ).

Evaluations. We used the aforementioned construction approach [9] to obtain full coverage under the order-based coverage criteria for the graphs discussed in Section 5.2.2. To this end, given a graph with $e$ nodes, we, in an attempt to make sure that every requested order can be covered, used the longest path length $l$ in the graph as the fixed-length. Note that this approach requires us to fix the length of the test cases to be generated. Consequently, the problem of covering different types of $t$-orders, independent of the actual coverage criterion used, was turned into a problem of computing a standard $t$-way covering array for $l$ options, each of which can take on $e$ settings. We used ACTS [29] to compute the required standard covering arrays. The experiments were repeated 5 times.

Table 15 presents the results we obtained. As the graphs got larger, since the number of settings for each option (i.e., the number of nodes $e$ in the graph) 


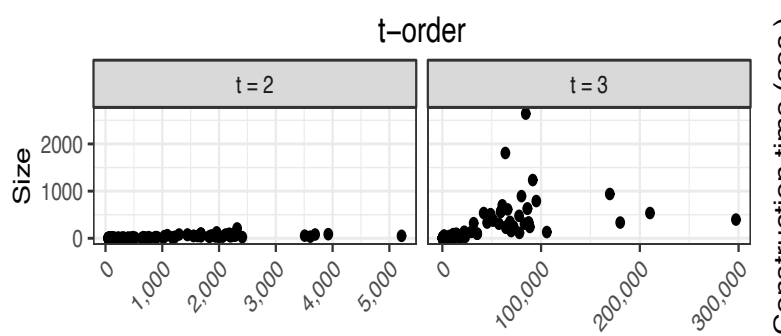

No of entities
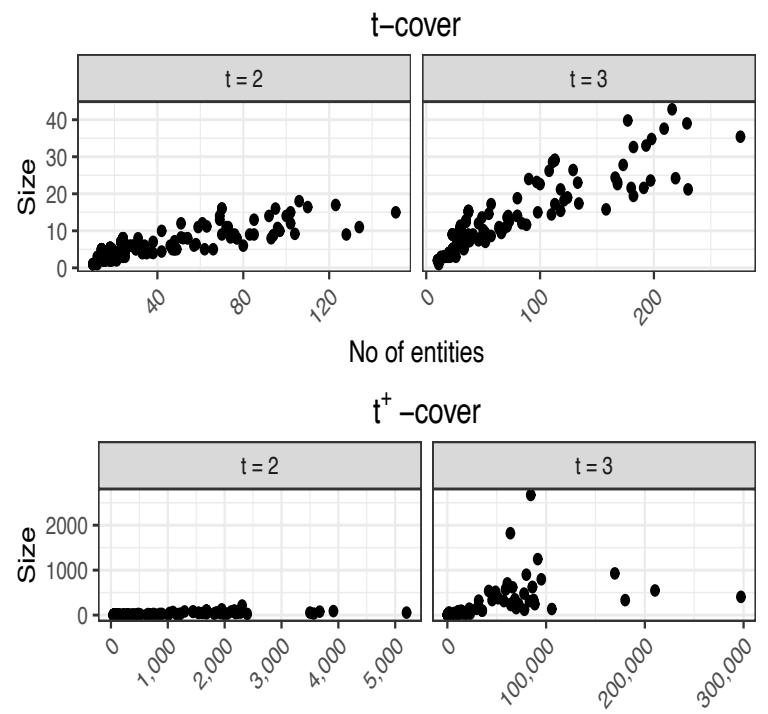

No of entities

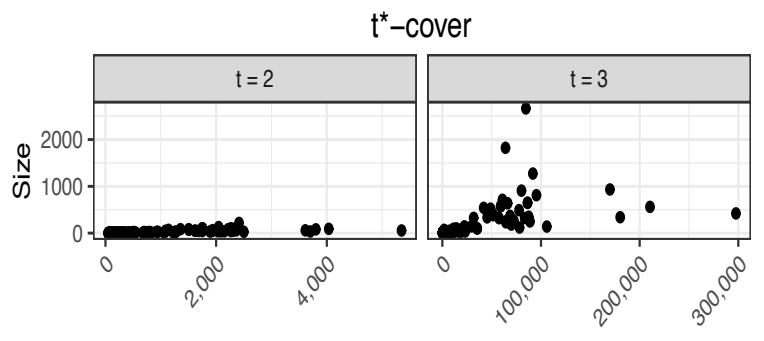

No of entities

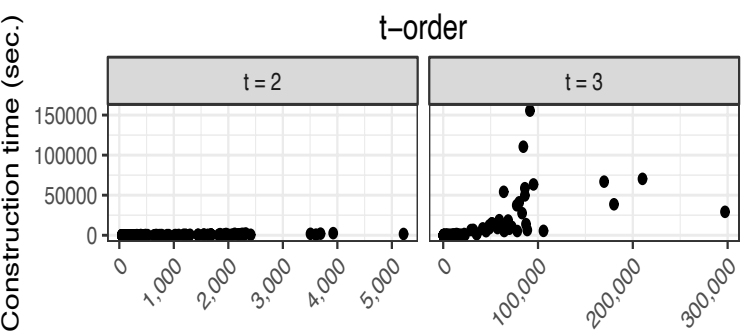

No of entities
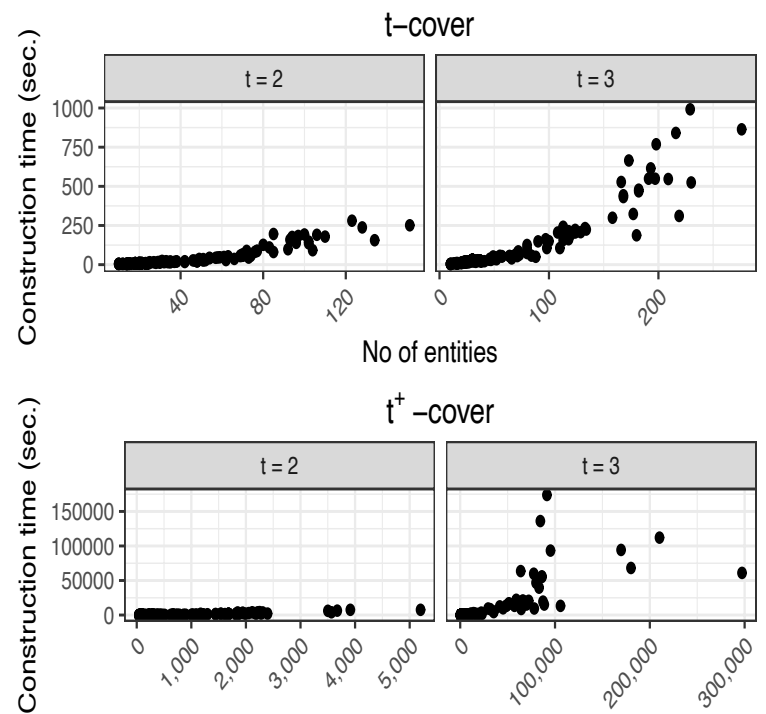

No of entities

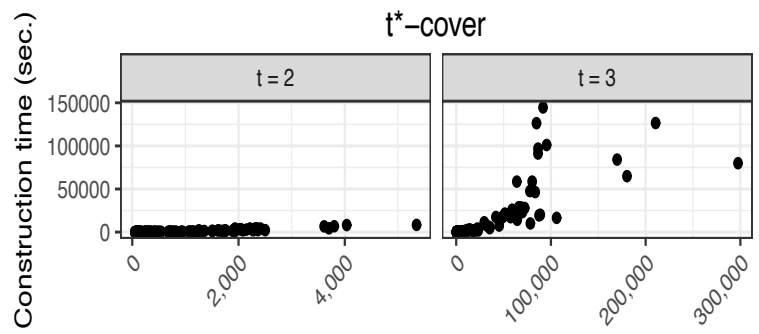

No of entities

Fig. 3: Results obtained for the coverage criteria given in Definitions 21-24. The horizontal axes represent the numbers of F-CIT entities to be covered, whereas the vertical axes depict either the average sizes of the F-CIT objects constructed or the average construction times (in seconds), depending on the graph. The experiments were repeated up to 5 times.

increased, it took increasingly longer times to compute the required covering arrays. This was indeed the case even for relatively small option counts (i.e., small values of $l$ ). As it was not feasible for us to generate all the required covering arrays, we employed a threshold value of 70 when $t=2$ and 30 when $t=3$ on the number of settings an option can have. This enabled us to cover all of the experimental setups, in which $e<70$ when $t=2$ and $e<30$ when $t=3$. These thresholds were chosen, such that the standard covering array constructor had one day to compute the requested object. Within the allocated time limits, we were able to generate standard covering arrays for $96.49 \%$ (165 out of 171) of the models when $t=2$ and for $66.67 \%$ (114 out of 171) of the models when $t=3$.

None of the test cases in the generated covering arrays, on the other hand, were valid. As no systematic approach is presented in [9] to take the reachability constraints enforced by the underlying graphs into account or to fix the invalid test cases in a post-mortem 
TABLE 13: Summary statistics for the construction times (in seconds) and the sizes of the $t$-way order-based F-CIT objects created for a) $t=2$ and (b) $t=3 \mathrm{~b}$. For each partition, the minimum, median, and maximum values encountered in the partition for the metrics in the columns are reported. The experiments were repeated up to 5 times.

\begin{tabular}{|c|c|c|c|c|c|c|c|}
\hline & part. & stat. & nodes & edges & entities & time & size \\
\hline \multirow{12}{*}{ 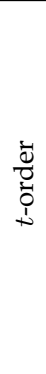 } & \multirow{3}{*}{1} & $\min$ & 12 & 12 & 31 & 2 & 1 \\
\hline & & med & 44 & 66 & 61 & 11 & 5 \\
\hline & & $\max$ & 46 & 67 & 71 & 18 & 6 \\
\hline & \multirow{3}{*}{2} & $\min$ & 14 & 13 & 78 & 3 & 1 \\
\hline & & med & 34 & 38 & 123 & 13 & 3 \\
\hline & & $\max$ & 70 & 99 & 224 & 41 & 13 \\
\hline & \multirow{3}{*}{3} & $\min$ & 35 & 40 & 227 & 18 & 2 \\
\hline & & med & 59 & 82 & 394 & 38 & 5 \\
\hline & & $\max$ & 172 & 248 & 1020 & 188 & 26 \\
\hline & \multirow{3}{*}{4} & $\min$ & 118 & 150 & 1037 & 116 & 11 \\
\hline & & med & 580 & 871 & 1936 & 725 & 54 \\
\hline & & $\max$ & 3604 & 6566 & 5215 & 2608 & 210 \\
\hline \multirow{12}{*}{ 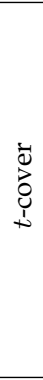 } & \multirow{3}{*}{1} & $\min$ & 12 & 12 & 10 & 3 & 1 \\
\hline & & med & 28 & 37 & 15 & 5 & 5 \\
\hline & & $\max$ & 44 & 66 & 17 & 9 & 5 \\
\hline & \multirow{3}{*}{2} & $\min$ & 19 & 23 & 18 & 6 & 2 \\
\hline & & med & 40 & 47 & 18 & 8 & 4 \\
\hline & & $\max$ & 54 & 73 & 21 & 11 & 5 \\
\hline & \multirow{3}{*}{3} & $\min$ & 23 & 28 & 23 & 7 & 3 \\
\hline & & med & 69 & 98 & 30 & 15 & 5 \\
\hline & & $\max$ & 460 & 620 & 52 & 36 & 12 \\
\hline & \multirow{3}{*}{4} & $\min$ & 112 & 150 & 54 & 29 & 5 \\
\hline & & med & 580 & 871 & 83 & 92 & 11 \\
\hline & & $\max$ & 3604 & 6566 & 151 & 399 & 21 \\
\hline \multirow{12}{*}{ 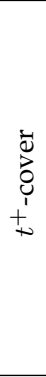 } & \multirow{3}{*}{1} & $\min$ & 12 & 12 & 30 & 12 & 1 \\
\hline & & med & 44 & 66 & 59 & 27 & 5 \\
\hline & & $\max$ & 46 & 67 & 68 & 39 & 6 \\
\hline & \multirow{3}{*}{2} & $\min$ & 16 & 18 & 73 & 27 & 1 \\
\hline & & med & 34 & 38 & 114 & 50 & 3 \\
\hline & & $\max$ & 71 & 111 & 222 & 141 & 13 \\
\hline & \multirow{3}{*}{3} & $\min$ & 35 & 40 & 241 & 114 & 2 \\
\hline & & med & 59 & 82 & 397 & 215 & 5 \\
\hline & & $\max$ & 172 & 248 & 1032 & 722 & 47 \\
\hline & \multirow{3}{*}{4} & $\min$ & 118 & 150 & 1089 & 695 & 11 \\
\hline & & med & 580 & 871 & 1936 & 2067 & 55 \\
\hline & & $\max$ & 3604 & 6566 & 5200 & 7559 & 213 \\
\hline \multirow{12}{*}{ 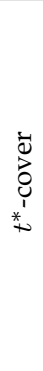 } & \multirow{3}{*}{1} & $\min$ & 12 & 12 & 42 & 15 & 1 \\
\hline & & med & 44 & 66 & 76 & 35 & 8 \\
\hline & & $\max$ & 46 & 67 & 86 & 53 & 8 \\
\hline & \multirow{3}{*}{2} & $\min$ & 16 & 18 & 88 & 35 & 2 \\
\hline & & med & 34 & 38 & 134 & 62 & 4 \\
\hline & & $\max$ & 70 & 99 & 240 & 151 & 14 \\
\hline & \multirow{3}{*}{3} & $\min$ & 35 & 40 & 242 & 118 & 2 \\
\hline & & med & 59 & 82 & 409 & 232 & 6 \\
\hline & & $\max$ & 172 & 248 & 1065 & 807 & 27 \\
\hline & \multirow{3}{*}{4} & $\min$ & 118 & 150 & 1084 & 644 & 14 \\
\hline & & med & 580 & 871 & 2029 & 2234 & 59 \\
\hline & & $\max$ & 3604 & 6566 & 5351 & 8383 & 218 \\
\hline
\end{tabular}

(a)

\begin{tabular}{|c|c|c|c|c|c|c|c|}
\hline & part. & stat. & nodes & edges & entities & time & size \\
\hline \multirow{12}{*}{ 㐫 } & \multirow{3}{*}{1} & $\min$ & 12 & 12 & 121 & 3 & 1 \\
\hline & & med & 28 & 37 & 312 & 34 & 9 \\
\hline & & $\max$ & 44 & 66 & 340 & 49 & 12 \\
\hline & \multirow{3}{*}{2} & $\min$ & 17 & 18 & 373 & 5 & 1 \\
\hline & & med & 46 & 67 & 526 & 38 & 8 \\
\hline & & $\max$ & 70 & 99 & 1023 & 81 & 19 \\
\hline & \multirow{3}{*}{3} & $\min$ & 34 & 38 & 1329 & 36 & 3 \\
\hline & & med & 54 & 69 & 3742 & 90 & 8 \\
\hline & & $\max$ & 172 & 248 & 14056 & 1612 & 98 \\
\hline & \multirow{3}{*}{4} & $\min$ & 99 & 128 & 15196 & 333 & 24 \\
\hline & & med & 580 & 864 & 65389 & 12517 & 347 \\
\hline & & $\max$ & 3604 & 6566 & 297308 & 155572 & 2643 \\
\hline \multirow{12}{*}{ 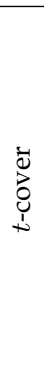 } & \multirow{3}{*}{1} & $\min$ & 12 & 12 & 10 & 4 & 1 \\
\hline & & med & 28 & 37 & 20 & 11 & 4 \\
\hline & & $\max$ & 54 & 69 & 24 & 20 & 9 \\
\hline & \multirow{3}{*}{2} & $\min$ & 20 & 24 & 25 & 15 & 3 \\
\hline & & med & 44 & 66 & 28 & 29 & 10 \\
\hline & & $\max$ & 50 & 72 & 30 & 33 & 11 \\
\hline & \multirow{3}{*}{3} & $\min$ & 23 & 28 & 31 & 17 & 5 \\
\hline & & med & 69 & 98 & 46 & 29 & 10 \\
\hline & & $\max$ & 152 & 219 & 73 & 84 & 17 \\
\hline & \multirow{3}{*}{4} & $\min$ & 112 & 150 & 80 & 49 & 11 \\
\hline & & med & 580 & 871 & 134 & 234 & 23 \\
\hline & & $\max$ & 3604 & 6566 & 276 & 1163 & 42 \\
\hline \multirow{12}{*}{ 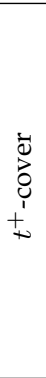 } & \multirow{3}{*}{1} & $\min$ & 12 & 12 & 121 & 18 & 1 \\
\hline & & med & 28 & 37 & 310 & 60 & 9 \\
\hline & & $\max$ & 44 & 66 & 330 & 82 & 12 \\
\hline & \multirow{3}{*}{2} & $\min$ & 17 & 18 & 371 & 39 & 1 \\
\hline & & med & 46 & 67 & 521 & 74 & 9 \\
\hline & & $\max$ & 70 & 99 & 1007 & 156 & 19 \\
\hline & \multirow{3}{*}{3} & $\min$ & 34 & 38 & 1327 & 160 & 3 \\
\hline & & med & 54 & 69 & 3735 & 277 & 8 \\
\hline & & $\max$ & 172 & 248 & 14034 & 2865 & 98 \\
\hline & \multirow{3}{*}{4} & $\min$ & 99 & 128 & 15185 & 1169 & 25 \\
\hline & & med & 580 & 864 & 65389 & 17037 & 349 \\
\hline & & $\max$ & 3604 & 6566 & 297294 & 173798 & 2672 \\
\hline \multirow{12}{*}{\begin{tabular}{l}
$\dot{0}$ \\
0 \\
0 \\
\multirow{1}{1}{} \\
$*$ \\
$+\infty$
\end{tabular}} & \multirow{3}{*}{1} & $\min$ & 12 & 12 & 136 & 22 & 1 \\
\hline & & med & 28 & 37 & 338 & 79 & 16 \\
\hline & & $\max$ & 44 & 66 & 346 & 142 & 18 \\
\hline & \multirow{3}{*}{2} & $\min$ & 17 & 18 & 401 & 49 & 3 \\
\hline & & med & 46 & 67 & 544 & 119 & 14 \\
\hline & & $\max$ & 70 & 99 & 1038 & 175 & 26 \\
\hline & \multirow{3}{*}{3} & $\min$ & 34 & 38 & 1373 & 182 & 4 \\
\hline & & med & 54 & 69 & 3763 & 394 & 15 \\
\hline & & $\max$ & 172 & 248 & 14144 & 3262 & 102 \\
\hline & \multirow{3}{*}{4} & $\min$ & 99 & 128 & 15253 & 1359 & 32 \\
\hline & & med & 580 & 864 & 65507 & 23023 & 368 \\
\hline & & $\max$ & 3604 & 6566 & 297570 & 144652 & 2665 \\
\hline
\end{tabular}

(b) manner, it is was not clear at all how to avoid and/or fix these invalid test cases.

\subsubsection{Applying F-CIT}

Given a graph $G=\left(V, E, v_{0}, v_{\perp}\right)$, which models the input space of the system under test, an F-CIT testable entity corresponds to a $t$-order, a consecutive-t-order, or a non-consecutive-t-order to be covered, depending on the the coverage criterion (Definitions 21-24). Then, an F-CIT test case corresponds to a path from $v_{0}$ to $v_{\perp}$ (Definition 17). Finally, the graph $G$, as it restricts the orders to be covered as well as the test cases to be generated, is expressed as the F-CIT model constraint $C$.

To this end, we encode the problem of finding a path from a source node to a sink node as a singlesource single-sink flow problem [44]. In particular, flow on an edge $\left(v_{i}, v_{j}\right) \in E$ (using the terminology of flow networks) is represented by a unique variable $e_{i j}$. From the perspective of finding a path, an edge $\left(v_{i}, v_{j}\right) \in E$ is either taken, i.e., $e_{i j}=1$, indicating that there is a flow on the edge, or not taken, i.e., $e_{i j}=0$, indicating that there is no flow on the edge: 
TABLE 14: Summary statistics for the construction times (in seconds) and the sizes of the 4-way order-based F-CIT objects computed. For each partition, the minimum, median, and maximum values encountered in the partition for the metrics in the columns are reported. Due to the cost, the experiments were repeated only once.

\begin{tabular}{|c|c|c|c|c|c|c|c|}
\hline & part. & stat. & nodes & edges & entities & time & size \\
\hline \multirow{12}{*}{ 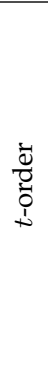 } & \multirow{3}{*}{1} & $\min$ & 12 & 12 & 274 & 1 & 1 \\
\hline & & med & 28 & 37 & 1059 & 34 & 9 \\
\hline & & $\max$ & 44 & 66 & 1432 & 61 & 10 \\
\hline & \multirow{3}{*}{2} & $\min$ & 17 & 18 & 1456 & 3 & 1 \\
\hline & & med & 46 & 67 & 1735 & 35 & 6 \\
\hline & & $\max$ & 51 & 73 & 2866 & 59 & 15 \\
\hline & \multirow{3}{*}{3} & $\min$ & 23 & 28 & 3189 & 6 & 1 \\
\hline & & med & 44 & 55 & 19424 & 51 & 6 \\
\hline & & $\max$ & 76 & 111 & 27601 & 786 & 191 \\
\hline & \multirow{3}{*}{4} & $\min$ & 41 & 49 & 29070 & 35 & 4 \\
\hline & & med & 99 & 135 & 118962 & 559 & 64 \\
\hline & & $\max$ & 578 & 804 & 4360399 & 92109 & 5742 \\
\hline \multirow{12}{*}{ 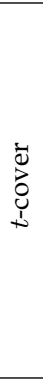 } & \multirow{3}{*}{1} & $\min$ & 12 & 12 & 10 & 2 & 1 \\
\hline & & med & 44 & 55 & 11 & 3 & 3 \\
\hline & & $\max$ & 2889 & 4956 & 14 & 23 & 7 \\
\hline & \multirow{3}{*}{2} & $\min$ & 17 & 18 & 15 & 3 & 2 \\
\hline & & med & 51 & 70 & 18 & 6 & 7 \\
\hline & & $\max$ & 1703 & 3121 & 21 & 19 & 9 \\
\hline & \multirow{3}{*}{3} & $\min$ & 19 & 23 & 23 & 9 & 5 \\
\hline & & med & 56 & 80 & 27 & 16 & 8 \\
\hline & & $\max$ & 2652 & 4147 & 39 & 67 & 15 \\
\hline & \multirow{3}{*}{4} & $\min$ & 59 & 82 & 40 & 19 & 6 \\
\hline & & med & 456 & 702 & 51 & 40 & 14 \\
\hline & & $\max$ & 2748 & 4264 & 143 & 197 & 26 \\
\hline \multirow{12}{*}{$\begin{array}{r}\ddot{d} \\
\grave{0} \\
\dot{0} \\
+ \\
+\infty\end{array}$} & \multirow{3}{*}{1} & $\min$ & 12 & 12 & 264 & 15 & 1 \\
\hline & & med & 28 & 37 & 1044 & 81 & 9 \\
\hline & & $\max$ & 44 & 66 & 1426 & 129 & 9 \\
\hline & \multirow{3}{*}{2} & $\min$ & 17 & 18 & 1438 & 43 & 1 \\
\hline & & med & 46 & 67 & 1724 & 103 & 6 \\
\hline & & $\max$ & 51 & 73 & 2833 & 140 & 15 \\
\hline & \multirow{3}{*}{3} & $\min$ & 23 & 28 & 3174 & 107 & 1 \\
\hline & & med & 44 & 55 & 19410 & 290 & 6 \\
\hline & & $\max$ & 76 & 111 & 27572 & 1145 & 191 \\
\hline & \multirow{3}{*}{4} & $\min$ & 41 & 49 & 29046 & 389 & 4 \\
\hline & & med & 112 & 154 & 149246 & 3071 & 70 \\
\hline & & $\max$ & 578 & 804 & 4360336 & 171050 & 5821 \\
\hline \multirow{12}{*}{$\begin{array}{l}\vec{D} \\
\overrightarrow{0} \\
\stackrel{0}{1} \\
\stackrel{*}{\sim}\end{array}$} & \multirow{3}{*}{1} & $\min$ & 12 & 12 & 274 & 20 & 1 \\
\hline & & med & 28 & 37 & 1059 & 93 & 11 \\
\hline & & $\max$ & 44 & 66 & 1432 & 123 & 13 \\
\hline & \multirow{3}{*}{2} & $\min$ & 17 & 18 & 1456 & 47 & 2 \\
\hline & & med & 46 & 67 & 1735 & 112 & 11 \\
\hline & & $\max$ & 51 & 73 & 2561 & 172 & 17 \\
\hline & \multirow{3}{*}{3} & $\min$ & 23 & 28 & 2866 & 119 & 4 \\
\hline & & med & 40 & 47 & 19424 & 259 & 7 \\
\hline & & $\max$ & 71 & 111 & 27214 & 1539 & 199 \\
\hline & \multirow{3}{*}{4} & $\min$ & 41 & 49 & 27601 & 414 & 10 \\
\hline & & med & 99 & 137 & 117735 & 2381 & 53 \\
\hline & & $\max$ & 578 & 804 & 4360399 & 189773 & 5858 \\
\hline
\end{tabular}

$$
e_{i j} \in\{0,1\} \text {. }
$$

Leaving cyclic graphs aside for the moment (which will be discussed later on in this section), to generate a test case, i.e., to form a flow from the source node $v_{0}$ to the sink node $v_{\perp}$, one of the outgoing edges of $v_{0}$ and one of the incoming edges of $v_{\perp}$ must be taken:
TABLE 15: Summary statistics for the construction times (in seconds) and the sizes of the standard covering arrays obtained by using the order-based construction approach presented in [9]. None of the test cases chosen by these standard covering arrays were valid. For each partition, the minimum, median, and maximum values encountered in the partition for the metrics in the columns are reported. The experiments were repeated 5 times.

\begin{tabular}{|c|c|c|c|c|c|c|}
\hline & part. & stats. & options & $\begin{array}{r}\text { settings } \\
\text { per options }\end{array}$ & time & size \\
\hline \multirow{12}{*}{ II } & \multirow{3}{*}{1} & $\min$ & 13 & 9 & 0.38 & 175 \\
\hline & & med & 28 & 12 & 0.64 & 336 \\
\hline & & $\max$ & 29 & 13 & 0.67 & 344 \\
\hline & \multirow{3}{*}{2} & $\min$ & 15 & 14 & 0.47 & 289 \\
\hline & & med & 31 & 17 & 0.86 & 561 \\
\hline & & $\max$ & 47 & 18 & 2.57 & 812 \\
\hline & \multirow{3}{*}{3} & $\min$ & 23 & 19 & 0.74 & 703 \\
\hline & & med & 39 & 22 & 3.22 & 1118 \\
\hline & & $\max$ & 129 & 37 & 80.44 & 3974 \\
\hline & \multirow{3}{*}{4} & $\min$ & 54 & 38 & 14.99 & 3474 \\
\hline & & med & 180 & 47 & 377.5 & 6282 \\
\hline & & $\max$ & 1153 & 63 & 43879.56 & 12171 \\
\hline \multirow{12}{*}{$\stackrel{\text { II }}{+}$} & \multirow{3}{*}{1} & $\min$ & 13 & 9 & 2.28 & 2594 \\
\hline & & med & 29 & 12 & 15.07 & 7014 \\
\hline & & $\max$ & 29 & 12 & 19.84 & 7014 \\
\hline & \multirow{3}{*}{2} & $\min$ & 15 & 13 & 3.8 & 6496 \\
\hline & & med & 28 & 13 & 19.1 & 8776 \\
\hline & & $\max$ & 32 & 15 & 90.78 & 14156 \\
\hline & \multirow{3}{*}{3} & $\min$ & 18 & 16 & 3.82 & 4096 \\
\hline & & med & 32 & 17 & 140.7 & 20501 \\
\hline & & $\max$ & 47 & 18 & 760.67 & 28240 \\
\hline & \multirow{3}{*}{4} & $\min$ & 23 & 19 & 62.53 & 24659 \\
\hline & & med & 33 & 21 & 410.34 & 38834 \\
\hline & & $\max$ & 62 & 27 & 11241.67 & 103923 \\
\hline
\end{tabular}

$$
\begin{aligned}
& \sum_{\left(v_{0}, v_{i}\right) \in E} e_{0 i}=1 \\
& \sum_{\left(v_{i}, v_{\perp}\right) \in E} e_{i \perp}=1 .
\end{aligned}
$$

Note that since the graph is acyclic, at most one of the incoming and at most one of the outgoing edges of a node can be taken, i.e., there can be a flow on at most one incoming and at most one outgoing edge.

The flow through node $i$ is then expressed as a constraint indicating that the amount of outgoing flow from $i$ is the same as the amount of incoming flow to $i$ :

$$
\sum_{\left(v_{k}, v_{i}\right) \in E} e_{k i}=\sum_{\left(v_{i}, v_{l}\right) \in E} e_{i l} \leq 1 .
$$

Note that the source and the sink nodes are exempt from (4) since there is no flow into the source node and no flow out of the sink node.

As an example, Figure 4 presents an encoding to compute a test case, i.e., a path from $v_{0}$ to $v_{\perp}$, for the graph given in Figure 2c.

To make sure that a specific order is covered by a test case, additional constraints are needed. More 


$$
\begin{gathered}
e_{01}, e_{12}, e_{13}, e_{14}, e_{15}, e_{24}, e_{34}, e_{45}, e_{5 \perp} \in\{0,1\} \\
e_{01}=1 \\
e_{5 \perp}=1 \\
e_{01}=e_{12}+e_{13}+e_{14}+e_{15} \\
e_{12}=e_{24} \\
e_{13}=e_{34} \\
e_{24}+e_{14}+e_{34}=e_{45} \\
e_{45}+e_{15}=e_{5 \perp}
\end{gathered}
$$

Fig. 4: Single-source single-sink encoding to find a path from the entry node $v_{0}$ to the exit node $v_{\perp}$ in the graph given in Figure 2c.

formally, to cover a $t$-order $\left[v_{i_{1}}, \ldots, v_{i_{t}}\right]$ in a graph $G=\left(V, E, v_{0}, v_{\perp}\right)$, the following additional constraints are needed:

$$
\sum_{\left(v_{k}, v_{i_{s}}\right) \in E} e_{k i_{s}}=1 \text { for } 1 \leq s \leq t,
$$

which indicate that all the nodes in the requested order must be visited. Since the graph is acyclic, the order of visit is guaranteed.

For example, to cover the 3 -order $\left[v_{1}, v_{4}, v_{5}\right]$ in Figure $2 c$, the encoding in Figure 4 needs to be extended with:

$$
\begin{gathered}
e_{01}=1 \\
e_{24}+e_{14}+e_{34}=1 \\
e_{45}+e_{15}=1 .
\end{gathered}
$$

To cover the same order $\left[v_{i_{1}}, \ldots, v_{i_{t}}\right]$ in a nonconsecutive manner, however, the following constraint is required in addition to (5):

$$
\sum_{1<s \leq t} e_{i_{s-1} i_{s}}<t-1,
$$

which ensures that the length of the path from $v_{i_{1}}$ to $v_{i_{t}}$ is at least $t$.

Going back to our running example, to cover $\left[v_{1}, v_{4}, v_{5}\right]$ in a non-consecutive manner, the following additional constraint is required on top of (6):

$$
e_{14}+e_{45}<2 \text {. }
$$

If, on the other hand, the $t$-order $\left[v_{i_{1}}, \ldots, v_{i_{t}}\right]$ needs to be covered in a consecutive manner, then the following constraints are needed instead of (5) and (7):

$$
e_{i_{s-1} i_{s}}=1 \text { for } 1<s \leq t,
$$

making sure that the edges between all the consecutive pairs of nodes in the order are taken.

For our running example in Figure 4, we would need the following additional constraints to cover $\left[v_{1}, v_{4}, v_{5}\right]$ in a consecutive manner:

$$
\begin{aligned}
& e_{14}=1 \\
& e_{45}=1 .
\end{aligned}
$$

For a given graph $G=\left(V, E, v_{0}, v_{\perp}\right)$, we have, therefore, defined the F-CIT model in this study as $M=<P, D, C>$, where $P$ is the set of variables, each of which represents a distinct edge in the graph; $D$ is a set of sets $\{0,1\}$, one per edge, indicating whether there is flow on the edge or not (i.e., whether the edge is taken or not); and $C$ is the model constraint capturing the reachability restrictions in the graph. More specifically, for a given graph, Equations 1-4 constitute the model constraint $C$. For example, Figure 4 presents the F-CIT model constraint created for the graph given in Figure 2c. Note that, given a graph, $C$ stays the same regardless of the testable entities to be covered. Each F-CIT testable entity then corresponds to an order to be covered (Definitions 21-24). In particular, to cover a $t$-order, Equation 5; to cover a $t$-order in a nonconsecutive manner, Equations 5 and 7; and to cover a $t$-order in a consecutive manner, Equation 9 needs to be used. As an example, Equation 6 presents the constraints to be used to cover the 3 -order $\left[v_{1}, v_{4}, v_{5}\right]$ in the graph given in Figure 2c. Similarly, Equations 6 and 8 are needed to cover the same 3-order in a nonconsecutive manner. And, Equation 10 is needed to cover it in a consecutive manner. Each F-CIT test case then corresponds to a path from the entry node $v_{0}$ to the exit node $v_{\perp}$ (Definition 17), covering a number of required orders.

Note that we have so far been concerned with directed acyclic graphs (DAGs). To work with cyclic graphs, we unroll the cycles $k$ times (for this work, $k=1$ ), which is a frequently used approach in bounded model checking [43]. To this end, we first convert a given graph to a regular expression [45], where all the Kleene plus operators are replaced by using the Kleene star operator, i.e., converting $a^{+}$to $a a^{*}$. We then replace all the Kleene stars in the expression using the bounded repetition operator, such that the respective strings can be repeated at most $k$ times, i.e., converting $a^{*}$ to $a\{0, k\}$. Finally, the resulting regular expression is converted back to a graph.

For this work, we used the Vcsn tool [46] to carry out these steps. More specifically, converting a graph to a regular expression and a regular expression to a graph were carried out by using a single Vcsn shell command. And, replacing the unbounded Kleene star operators by bounded repetition operators was performed by another shell command using the replace string-replacement utility. 
o graph

edge $(\mathrm{v} 0, \mathrm{v} 1)$.

edge (v1, v2).

$\div$..

\% 'reaches' definitions

reaches $(A, B)$ :- edge $(A, B)$.

reaches $(A, B)$ :- edge $(A, C)$, reaches $(C, B)$.

$\div 3$-orders

$\operatorname{order}(A, B, C)$ :- $\operatorname{reaches}(A, B)$, reaches $(B, C)$.

\% consecutive-3-orders

consec $(A, B, C):-\operatorname{edge}(A, B)$, edge $(B, C)$.

- non-consecutive-3-orders

nonconsec $(A, B, C):-\operatorname{reaches}(A, X), X !=B$, reaches $(X, B)$, reaches $(B, C)$.

nonconsec $(A, B, C):-\operatorname{reaches}(A, B)$, reaches $(B, X), X !=C$ reaches $(X, C)$.

Fig. 5: An example ASP encoding for determining the valid consecutive, nonconsecutive, and regular 3-orders.

After having an acyclic graph, we used ASP (Answer Set Programming) [47], [48] to determine the different types of $t$-orders to be covered. Note that this step could also have been carried out by using reachabilitybased graph algorithms. We, however, chose to use ASP because, being a declarative logic programming paradigm, it was a perfect match for the task at hand. We were even able to provide whole code segments in the paper (e.g., Figure 5) to demonstrate the effort involved in the development

Figure 5 presents an example ASP encoding to determine the consecutive, non-consecutive, and regular 3 -orders. Below, we explain the encoding in a nutshell with no intention to introduce ASP. For more details about ASP, the interested reader may refer to an introduction [49] or a book [50].

A DAG is expressed by using edge ( . . ) facts. There is a path from node $A$ to node $B$, i.e., $A$ reaches $B$ or $B$ is reachable from $A$, if there is an edge from $A$ to $B$ (i.e., edge $(A, B)$ holds) or there is an edge from $A$ to $\mathrm{C}$ and $\mathrm{B}$ is reachable from $\mathrm{C}$ :

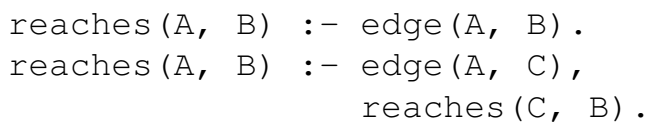

Then, $[A, B, C]$ is a valid 3-order, i.e., $\operatorname{order}(A, B, C)$, if $A$ reaches $B$ and $B$ reaches C:

$$
\begin{aligned}
\operatorname{order}(A, B, C):- & \operatorname{reaches}(A, B), \\
& \text { reaches }(B, C) .
\end{aligned}
$$

For a 3-order $[\mathrm{A}, \mathrm{B}, \mathrm{C}]$ to be covered in a consecutive manner, there should be an edge from $A$ to $B$ and edge from $B$ to $C$ :

$$
\begin{array}{r}
\operatorname{consec}(A, B, C):- \\
\operatorname{edge}(A, B), \\
\operatorname{edge}(B, C) .
\end{array}
$$

And, for the same order to be covered in a nonconsecutive manner, $B$ should be reachable from $A$ via another node or $\mathrm{C}$ should be reachable from $\mathrm{B}$ via another node:

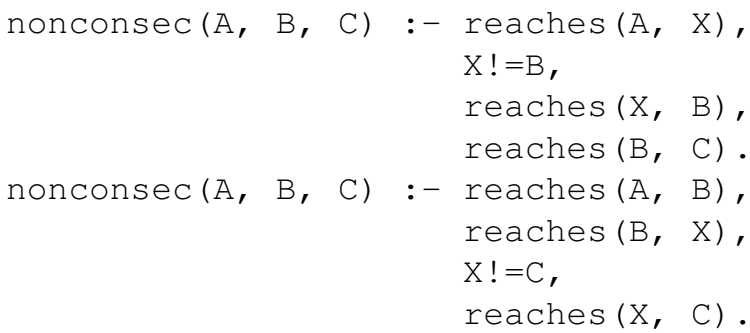

Note that this encoding can trivially be extended to determine $t$-orders for any strength $t$.

Cost. All told, developing a generic Python script to unroll the cycles in a given graph using $\mathrm{V}$ csn took about 2 hours, which was mostly spent for writing procedures to match the input and output formats of Vcsn. Similarly, developing a generic Python script to automatically generate the ASP encodings for determining different types of $t$-orders to be covered, such as the one given in Figure 5, took about another 2 hours. Integrating a CSP solver, namely Sugar [51], with the constructors (as also discussed in Section 5.3.4) took less than 1 hour.

Evaluations. To evaluate the proposed approach, we first used our F-CIT constructors to compute $t$-way $(t=\{2,3\})$ order-based F-CIT objects for the graphs discussed in Section 5.2.2. The experiments were repeated up to 5 times; $94 \%$ of the experiments with the best-performing F-CIT constructor (i.e., cover-andgenerate) were repeated exactly 5 times. By construction, all the test cases selected by the F-CIT objects computed were valid and all these F-CIT objects achieved full coverage under the respective coverage criterion.

As was the case with the previous study (Section 5.1), the cover-and-generate constructor performed generally better than the generate-and-cover constructor. Therefore, we ran the generate-and-cover constructor with a time-out period of one day, while letting the cover-and-generate constructor run to completion. For $93.20 \%$ (1275 out of 1368) of the experimental setups, the generate-and-cover constructor computed the requested F-CIT objects within the allocated time limits. For these setups, the cover-and-generate constructor reduced the sizes by an average of $65.86 \%$ and $60.79 \%$, while at the same time reducing the construction times by an average of $72.24 \%$ and $77.03 \%$ when $t=2$ and 3 , respectively. We, therefore, focus on the results obtained from the cover-and-generate constructor in the remainder of this section.

Figure 3 presents the results obtained from the coverand-generate constructor and Table 13 provides some summary statistics. As expected, the coverage criteria listed in the order of increasing number of entities they required to cover, were: $t$-cover, $t^{+}$-cover, $t$-order, 
and $t^{*}$-cover. These criteria respectively marked an average of $38.50,665.87,672.37$, and 704.36 entities for coverage when $t=2$; and an average of $61.73,21678.98$, 21685.99 , and 21740.71 entities when $t=3$.

The sizes of the order-based F-CIT objects as well their construction times tended to be correlated with the number of entities to be covered. Overall, the minimum, the average, and the maximum sizes of the FCIT objects created were $1,17.28$, and 220, respectively, when $t=2$; and $1,54.27$, and 2672 when $t=3$. And the construction times for these objects respectively were $2.57,461.32$, and 4156.25 seconds when $t=2$; and 3.24 , 2568.12 , and 136116.19 seconds when $t=3$.

Another trend we observed was that although the numbers of entities to be covered by the $t$-order criterion were similar to those to be covered by the $t^{+}$-cover and $t^{*}$-cover criteria, covering the latter set of entities took longer than covering the former set of entities. The average constructions times were 251.29, 751.03, and 806.92 seconds for $t$-order, $t^{+}$-cover, and $t^{*}$-cover criteria, respectively, when $t=2$; and 6885.59, 9508.46, and 10626.21 seconds when $t=3$. We believe that this was because of the additional constraints to be satisfied to make sure that the requested orders are covered in a non-consecutive manner (i.e., need for solving the constraints in Equation 7 on top of Equation 5).

Computing 4-way order-based F-CIT objects. Last but not least, we ran our cover-and-generate constructor for $t=4$ with a time-out period of 200 hours. For $88.01 \%$ ( 602 out of 684 ) of the experimental setups, the constructor was able to generate the requested F-CIT objects within the allocated time limits, whereas for the remaining $11.99 \%$ of the setups, it timed out.

Table 14 presents the results we obtained. Overall, the minimum, the average, and the maximum sizes of the 4-way order-based F-CIT objects computed were $1,136.42$, and 5858, respectively. And the construction times for these objects respectively were 1.87, 4522.53, and 189773.20 seconds.

\subsubsection{Discussion}

Note that given a graph, there are different approaches for solving the problem of finding a path covering certain sequences of nodes. In this study, however, our goal was to demonstrate that there is at least one solution, which can be expressed in F-CIT. For example, instead of using a constraint solver, one can use a model checker and formulate the same problem as a property stating that there is no path covering the requested orders. A counter example (if any) would then be a test case covering the orders. Similarly, one can even develop a special purpose constraint solver, which uses graph-based reachability algorithms, to determine whether a given set of orders can appear on a single path. These solutions would all work with F-CIT as long as the underlying solver supports the single primitive solve as discussed in Section 4.

\subsection{Study 3: Usage-Based CIT}

An electronics company has approached us to improve their CIT-based testing practices. In particular, they were interested in testing the Internet connectivity feature of a consumer device, which they market in dozens of countries. The end-users of this device can customize the aforementioned feature by using 9 configuration options, which have 308, 280, 154, 82, 58, $41,6,3$, and 2 settings, respectively. Since there is no system-wide constraint, all possible configurations (i.e., all possible combinations of option settings) are valid. All told, these options constitute a space of more than 90 billion valid configurations.

The company provided us with 526691 real configurations that they collected from the field during the month of May in 2016. Each configuration was obtained from a different consumer device and there were a total of 37503 distinct configurations, i.e., some configurations were used by multiple costumers.

Historically, configuration-related failures in this system have often been caused by the faulty interactions among the configuration options. However, exhaustive testing of neither the whole configuration space nor the distinct configurations seen in the field, is desirable for the company. Due to legal and privacy concerns, we are not able to provide further details.

\subsubsection{Coverage criterion}

We first attempted to create standard covering arrays for the scenario at hand (see Section 5.3.3 for more information). It turned out that the smallest covering array we could generate was a 2-way covering array of size 86241 . It is, however, quite difficult to justify the use of all these configurations for testing when one knows that the total number of distinct configurations used in the field is 37503. Had the company had enough resources (i.e., time and computing platforms) to test all the distinct configurations in the field, they would have done it.

We, therefore, defined two novel coverage criteria, namely $K_{\text {seen }}$ and $K_{\text {weighted }}$, based on the idea that when testing all $t$-tuples is not feasible, one should at the very least, consider testing the $t$-tuples appearing in the field. We call this usage-based CIT.

Definition 25. The seen-t-way coverage criterion $K_{\text {seen }}$ takes as input a set of configurations $T$, a coverage strength $t$, and a cutoff frequency in $[0,1)$, and mark for coverage all the $t^{\prime}$-tuples $\left(1 \leq t^{\prime} \leq t\right)$ appearing in $T$, the frequencies of which are greater than the cutoff frequency.

The frequency of a tuple is computed as follows:

Definition 26. Given a set of configurations $T$, the frequency of a tuple is the ratio of the number of configurations in $T$, in which the tuple appear, to the total number of configurations in $T$. 
Note that, when the frequency cutoff is $0, K_{\text {seen }}$ selects all the $t^{\prime}$-tuples $\left(1 \leq t^{\prime} \leq t\right)$ appearing in $T$.

$K_{\text {seen }}$ can further be extended to obtain variable strength coverage by using a weighted sum of the frequencies, where the weight of a tuple is defined as follows:

Definition 27. Given a set of configurations $T$, the weight of a tuple is the ratio of the number of times the tuple appears in $T$ to the total number of tuples in $T$.

Note that computing the denominator in Definition 27 does not require to explicitly enumerate all possible tuples appearing in $T$. More specifically, since the number of tuples in a given configuration of $k$ options is $2^{k}-1$, the total number of tuples in $T$ (thus the denominator) is $|T|\left(2^{k}-1\right)$.

Definition 28. The weighted-t-way coverage criterion $K_{\text {weighted }}$ takes as input a set of configurations $T$, a coverage strength $t$, and $a$ cutoff weight in $(0,1]$, and mark for coverage a minimal set of $t^{\prime}$-tuples $\left(1 \leq t^{\prime} \leq t\right)$, the total weight of which is greater than or equal to the given cutoff weight.

To determine the tuples to be covered by this criterion, all the $t^{\prime}$-tuples $\left(1 \leq t^{\prime} \leq t\right)$ appearing in $T$ are sorted by the descending order of their weights. Then, the minimum number tuples from the top of the list are selected, such that the total weight of the selected tuples is greater than or equal to the cutoff weight. Note that the $K_{\text {weighted }}$ criterion with the cutoff weight of 1 can be satisfied by selecting all the distinct configurations in $T$.

\subsubsection{Study setup}

For the evaluations, we used the aforementioned subject application with 9 configuration options, which had $308,280,154,82,58,41,6,3$, and 2 settings, respectively, together with the 526691 real configurations collected from the field, out of which 37503 were distinct.

All the experiments were carried out on the same Google Cloud platform with the previous two studies (Sections 5.1 and 5.2).

\subsubsection{Applying standard CIT}

Modeling. We first attempted to create standard covering arrays of various strengths by using a number of well-known covering array constructors, namely Jenny [52], PICT [53], and ACTS [29].

The very first thing we observed was that although we had a small number of configuration options (only 9 ), due to the large number of settings some of these options had, many of the existing covering array constructors failed to generate the requested covering arrays. For example, we were not even able to model the configuration space in Jenny, because it turned out
Jenny employs the letters of the English alphabet to represent the settings of a configuration option, limiting the maximum number of settings that an option can have to 52 (the number of capital and lowercase letters in the English alphabet). On the other hand, PICT, which is specifically designed for scalability [53], was able to generate a 2-way covering array of size 86241 in 100 seconds. It, however, failed to generate a 3-way covering array in 10 days, after which we terminated the process. Whereas ACTS was able to generate a 2-way covering array of size 86255 in 16 seconds and a 3-way covering array of size 13283730 in 1887 seconds (about 32 minutes). However, when we attempted to generate 4-way covering arrays, ACTS crashed after a while with some memory-related errors.

Note that given a usage-based coverage criterion, neither the tuples to be covered nor the tuples not to be covered can be expressed as constraints in standard constructors in an attempt to selectively determine what to cover and what not to cover. This is because constraints in standard constructors are globally enforced, i.e., all of the test cases selected must satisfy all of the constraints. Therefore, expressing a tuple, which is selected by a given coverage criterion, as a constraint to indicate that the tuple needs to be covered, will enforce the same tuple to appear in all of the selected configurations. Since this can prevent conflicting tuples from being covered, no covering array can be created. Similarly, expressing a tuple, which is not selected by a given coverage criterion, as a constraint to indicate that the tuple needs to be avoided, can also prevent the creation of a covering array. It may not, for example, be possible to assign values to certain model parameters due to some invalidated tuple combinations.

An alternative approach might be to express tuples that are not needed to be covered as soft constraints, which mark combinations of parameter values that are permitted, but not desirable [16]. However, when the tuples to be covered is a small fraction of all the tuples, the number of soft constraints can get quite large, which can in turn cause performance and scalability issues. For example, in our experiments, $99.90 \%$ of all the tuples (of strength up to and including a given value of $t$ ), on average, did not need to be covered. In other words, had soft constraints been used to express these need-not-to-be-covered tuples, the number of constraints would have been as high as 4.7 trillion in some experiments. We couldn't experiment with this approach, because none of the standard constructors that we have access to, supported soft constraints.

Evaluations. All told, the size of the smallest standard covering array that we could generate was larger than the number of distinct configurations seen in the field, which rendered the use of standard covering arrays in this context hard to justify. 
TABLE 16: Statistics about the $K_{\text {seen }}$ coverage obtained by standard covering arrays. The columns, respectively, report the frequency cutoff values, the numbers of testable entities to be covered, and the numbers of testable entities covered by the standard 2-way and 3-way covering arrays created for the study.

\begin{tabular}{|c|c|c|c|c|c|c|c|c|c|c|c|c|c|c|c|}
\hline \multirow[b]{3}{*}{ cutoff } & \multicolumn{3}{|c|}{$t=2$} & \multicolumn{3}{|c|}{$t=3$} & \multicolumn{3}{|c|}{$\mathrm{t}=4$} & \multicolumn{3}{|c|}{$t=5$} & \multicolumn{3}{|c|}{$\mathrm{t}=6$} \\
\hline & \multirow{2}{*}{$\begin{array}{r}\text { no of } \\
\text { entities }\end{array}$} & \multicolumn{2}{|c|}{$\begin{array}{c}\text { \% covered } \\
\text { by standard CAs }\end{array}$} & \multirow{2}{*}{$\begin{array}{r}\text { no of } \\
\text { entities }\end{array}$} & \multicolumn{2}{|c|}{$\begin{array}{c}\% \text { covered } \\
\text { by standard CAs }\end{array}$} & \multirow{2}{*}{$\begin{array}{r}\text { no of } \\
\text { entities }\end{array}$} & \multicolumn{2}{|c|}{$\begin{array}{c}\% \text { covered } \\
\text { by standard CAs }\end{array}$} & \multirow{2}{*}{$\begin{array}{r}\text { no of } \\
\text { entities }\end{array}$} & \multicolumn{2}{|c|}{$\begin{array}{c}\% \text { covered } \\
\text { by standard CAs }\end{array}$} & \multirow{2}{*}{$\begin{array}{r}\text { no of } \\
\text { entities }\end{array}$} & \multicolumn{2}{|c|}{$\begin{array}{c}\% \text { covered } \\
\text { by standard CAs }\end{array}$} \\
\hline & & 2-way & 3-way & & 2-way & 3-way & & 2-way & 3-way & & 2-way & 3-way & & 2-way & 3-way \\
\hline 0.5 & 4 & 100 & 100 & 4 & 100 & 100 & 4 & 100 & 100 & 4 & 100 & 100 & 4 & 100 & 100 \\
\hline 0.25 & 20 & 100 & 100 & 25 & 100 & 100 & 26 & 100 & 100 & 26 & 100 & 100 & 26 & 100 & 100 \\
\hline 0.2 & 34 & 100 & 100 & 44 & 100 & 100 & 49 & 100 & 100 & 50 & 98 & 100 & 50 & 98 & 100 \\
\hline 0.15 & 54 & 100 & 100 & 89 & 97 & 100 & 100 & 94 & 100 & 101 & 93 & 100 & 101 & 93 & 100 \\
\hline 0.1 & 80 & 100 & 100 & 164 & 90 & 100 & 235 & 78 & 100 & 264 & 70 & 97 & 269 & 69 & 96 \\
\hline 0.05 & 200 & 100 & 100 & 474 & 85 & 100 & 734 & 67 & 96 & 900 & 56 & 87 & 971 & 51 & 81 \\
\hline 0.04 & 240 & 100 & 100 & 601 & 84 & 100 & 964 & 65 & 95 & 1204 & 53 & 85 & 1318 & 48 & 79 \\
\hline 0.03 & 299 & 100 & 100 & 811 & 80 & 100 & 1395 & 58 & 94 & 1811 & 46 & 82 & 2001 & 42 & 75 \\
\hline 0.02 & 422 & 100 & 100 & 1281 & 76 & 100 & 2382 & 53 & 92 & 3256 & 39 & 79 & 3705 & 35 & 71 \\
\hline 0.01 & 669 & 100 & 100 & 2201 & 74 & 100 & 4475 & 47 & 90 & 6585 & 33 & 74 & 7825 & 28 & 63 \\
\hline 0.005 & 1056 & 100 & 100 & 3751 & 71 & 100 & 7949 & 44 & 89 & 12028 & 30 & 71 & 14634 & 25 & 59 \\
\hline 0.001 & 2652 & 100 & 100 & 11604 & 67 & 100 & 27890 & 39 & 85 & 45599 & 25 & 65 & 57741 & 19 & 53 \\
\hline 0 & 22554 & 100 & 100 & 182952 & 42 & 100 & 658825 & 11 & 67 & 1240182 & 1 & 27 & 1321685 & 0 & 4 \\
\hline
\end{tabular}

TABLE 17: Statistics about the $K_{\text {weighted }}$ coverage obtained by standard covering arrays. The columns, respectively, report the weight cutoff values, the numbers of testable entities to be covered, and the numbers of testable entities covered by the standard 2-way and 3-way covering arrays created for the study.

\begin{tabular}{|c|c|c|c|c|c|c|c|c|c|c|c|c|c|c|c|}
\hline \multirow[b]{3}{*}{ cutoff } & \multicolumn{3}{|c|}{$t=2$} & \multicolumn{3}{|c|}{$t=3$} & \multicolumn{3}{|c|}{$t=4$} & \multicolumn{3}{|c|}{$t=5$} & \multicolumn{3}{|c|}{$t=6$} \\
\hline & \multirow{2}{*}{$\begin{array}{r}\text { no of } \\
\text { entities }\end{array}$} & \multicolumn{2}{|c|}{$\begin{array}{c}\% \text { covered } \\
\text { by standard CAs }\end{array}$} & \multirow{2}{*}{$\begin{array}{r}\text { no of } \\
\text { entities }\end{array}$} & \multicolumn{2}{|c|}{$\begin{array}{c}\% \text { covered } \\
\text { by standard CAs }\end{array}$} & \multirow{2}{*}{$\begin{array}{r}\text { no of } \\
\text { entities }\end{array}$} & \multicolumn{2}{|c|}{$\begin{array}{c}\% \text { covered } \\
\text { by standard CAs }\end{array}$} & \multirow{2}{*}{$\begin{array}{r}\text { no of } \\
\text { entities }\end{array}$} & \multicolumn{2}{|c|}{$\begin{array}{c}\% \text { covered } \\
\text { by standard CAs }\end{array}$} & \multirow{2}{*}{$\begin{array}{r}\text { no of } \\
\text { entities }\end{array}$} & \multicolumn{2}{|c|}{$\begin{array}{c}\% \text { covered } \\
\text { by standard CAs }\end{array}$} \\
\hline & & 2-way & 3-way & & 2-way & 3-way & & 2-way & 3-way & & 2-way & 3-way & & 2-way & 3-way \\
\hline 0.70 & 332 & 100 & 100 & 2171 & 74 & 100 & 8037 & 44 & 89 & 18518 & 28 & 69 & 29272 & 22 & 56 \\
\hline 0.75 & 426 & 100 & 100 & 2951 & 72 & 100 & 11240 & 42 & 87 & 26467 & 27 & 68 & 42694 & 21 & 54 \\
\hline 0.80 & 566 & 100 & 100 & 4117 & 70 & 100 & 16252 & 41 & 87 & 39309 & 25 & 66 & 63498 & 19 & 52 \\
\hline 0.85 & 793 & 100 & 100 & 6051 & 69 & 100 & 24798 & 39 & 86 & 60222 & 23 & 64 & 98886 & 17 & 50 \\
\hline 0.90 & 1185 & 100 & 100 & 9675 & 68 & 100 & 40330 & 37 & 85 & 101015 & 21 & 62 & 169254 & 15 & 47 \\
\hline 0.95 & 2073 & 100 & 100 & 17826 & 64 & 100 & 79330 & 33 & 83 & 211007 & 17 & 58 & 368650 & 12 & 43 \\
\hline
\end{tabular}

To further demonstrate that obtaining full coverage in an efficient and effective manner under the usagebased coverage criteria is a non-trivial task, Tables 1617 report the coverage percentages obtained by the standard covering arrays generated in this study. In particular, when $t>3$ with $K_{\text {seen }}$, the standard 2- and 3 -way covering arrays did not guarantee to cover all the requested tuples. For example, when cutoff $=0.001$, only $39 \%(85 \%), 25 \%(65 \%)$, and $19 \%(53 \%)$ of all the required tuples for $t=4,5$, and 6 under $K_{\text {seen }}$, were covered by the standard 2-way (3-way) covering arrays (Table 16). Similarly, the standard covering arrays did not guarantee to cover all the tuples requested by $K_{\text {weighted }}$ either, especially for large values of coverage strength and weight cutoff values. For example, when $t=6$ and cutoff $=0.95$ only $12 \%$ and $43 \%$ of all the required tuples were covered by the standard 2-way and 3-way covering arrays, respectively (Table 17).

\subsubsection{Applying F-CIT}

Modeling. We have defined the F-CIT model as $\quad M=<P, D, C\rangle$, where $P=\left\{o_{1}, \ldots, o_{9}\right\}$, $D=\{\{1 . .308\},\{1 . .280\},\{1 . .154\},\{1 . .82\},\{1 . .58\},\{1 . .41\}$, $\{1 . .6\},\{1 . .3\},\{1,2\}\}$, and $C:$ true, indicating that all possible configurations were valid.

Each F-CIT testable entity then naturally corresponded to a tuple selected by the coverage criterion $K_{\text {seen }}$ or $K_{\text {weighted }}$, which was expressed as a constraint over finite sets. For example, the 3 -tuple $\left(o_{1}=204, o_{5}=12, o_{9}=1\right)$ was expressed as $o_{1}=204 \wedge o_{5}=12 \wedge o_{9}=1$. Note that the very same approach can readily be used to define and compute standard $t$-way covering arrays as F-CIT objects by expressing all valid $t$-tuples as F-CIT testable entities.

Consequently, any solver that works with logical operators, such as $\wedge$ (AND), and equality constraints over finite sets, such as $o_{1}=204$, including the commonplace SAT and CSP solvers [54], [55], can be used with the F-CIT constructors compute the F-CIT objects satisfying the $K_{\text {seen }}$ and $K_{\text {weighted }}$ criteria.

Indeed, being able to work with any type of constraints as long as an appropriate solver is provided, improves the flexibility of F-CIT. To demonstrate that this feature also enables the use of domain- and/or application-specific solvers, we have implemented a quite simple solver for this study, instead of trivially using an existing SAT or CSP solver.

Algorithm 3 presents the aforementioned solver. It simply determines whether a given set of tuples $E$ can be accommodated together in a single configuration or not. In particular, it marks $E$ as satisfiable as long as the option settings appearing in $E$ do not contradict with each other (lines 7-8).

Cost. All told, developing a generic script to determine the tuples (i.e., the testable entities) selected by the $K_{\text {seen }}$ and $K_{\text {weighted }}$ coverage criteria for any configuration space model, coverage strength, and cutoff value, took less than 2 hours. And, implementing the solver in Algorithm 3 and integrating it with the F-CIT constructors took less than 1 hour. To further demon- 


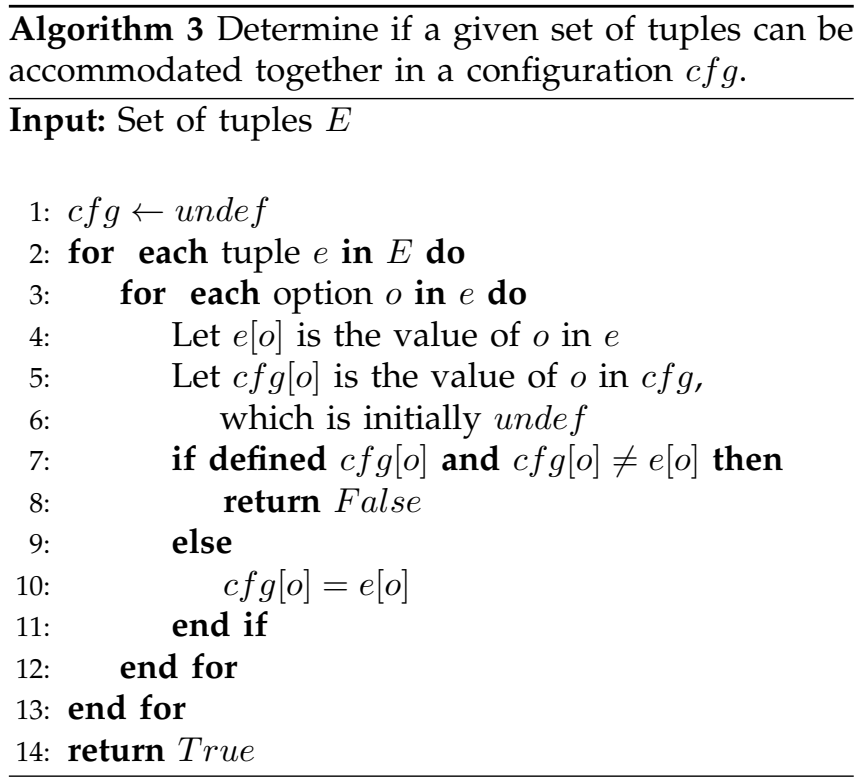

strate the flexibility of F-CIT, we have also integrated our constructors with a CSP solver (namely, Sugar [51]) to solve exactly the same set of constraints. Interestingly enough, it took about the same time (less than 1 hour) for us to do that as we needed to implement a simple procedure to match the input format of the solver. The implementation was done in Python.

Evaluations. To evaluate the proposed approach, we carried out a series of experiments. In these experiments, we used the cover-and-generate and generateand-cover constructors given in Algorithms 1 and 2 to compute F-CIT objects of various strengths. Since the cover-and-generate constructor performed generally better than the generate-and-cover constructor, the experiments with the latter constructor were repeated up to 3 times and with a time-out period of one day for each repetition to keep the cost of the experiments under control. The experiments with the former constructor, on the other hand, were repeated 100 times to evaluate the sensitivity of the proposed approach to the order, in which the testable entities are processed, except for the experimental setups where the frequency cutoff was 0 and $t>2$, which were repeated only once, to keep the cost under further control. In all the experiments, the orders were randomly generated by shuffling the testable entities to be covered.

Evaluating the $K_{\text {seen }}$ coverage criterion. Table 18 summarizes the results we obtained for the $K_{\text {seen }}$ coverage criterion. We first observed that the F-CIT constructors, especially the cover-and-generate constructor, were scaled to obtain full coverage under $K_{\text {seen }}$ for various values of $t$ up to and including 6 , even when the frequency cutoff was 0 . As a matter of fact, we chose to stop at the strength of 6 , because, in the presence of 9 options, increasing the strength any further was quickly becoming exhaustive testing, which, in this context, is the same as testing all the distinct configurations seen in the field.

We then observed that the cover-and-generate constructor performed generally better than the generateand-cover constructor in reducing both the covering array sizes and construction times. More specifically, the cover-and-generate constructor reduced the sizes by an average of $65.62 \%, 82.55 \%, 86.35 \%, 88.25 \%$, and $88.16 \%$ while at the same time reducing the construction times by an average of $96.35 \%, 97.20 \%, 97.88 \%, 97.72 \%$, and $97.32 \%$ when $t=2,3,4,5$, and 6 , respectively. We, therefore, focus on the results obtained from the cover-and-generate constructor in the remainder of this section.

When $t \leq 3$ and cutoff $=0$, i.e., when all the $t$-tuples seen in the field are required to be covered, the sizes of the F-CIT objects generated by the cover-and-generate constructor, were smaller than the number of distinct configurations seen in the field, i.e., 37503. More specifically, the average sizes were 3625.24 and 24971.00 with the average construction times of 205.55 and 7216.62 seconds for $t=2$ and 3 , respectively (Table 18). When $t>3$ and cutoff $=0$, however, the F-CIT objects had more than 37503 configurations, on average (Table 18).

In reality, when testing all the $t$-tuples seen in the field is still not practical due to the cost, the cutoff parameters of the usage-based coverage criteria can be utilized to select a weighted fraction of the tuples for testing. For example, when the frequency cutoff was set to 0.001 with $K_{\text {seen }}$, i.e., when the tuples that appeared in at least one thousandth of the configurations seen in the field were to be covered, the average sizes of the F-CIT objects became 327.31, 817.66, 1124.70, 1123.06, and 1015.85 when $t=2,3,4,5$, and 6 , respectively.

All the results we obtained under different coverage strengths and cutoff values can be found in Table 18 . For a fixed strength, as the cutoff increased, the number of testable entities as well as the size of the F-CIT objects tended to decrease. For example, when $t=6$, the average sizes of the F-CIT objects were 1015.85, $206.63,95.96,46.23$, and 5.84 for cutoff $=0.001,0.005$, $0.01,0.02$, and 0.1 , respectively. For a fixed cutoff, as the strength increased, on the other hand, although the number of testable entities to be covered increased, this did not necessarily cause an increase in the sizes of the F-CIT objects computed. For example, when cutoff $=0.005$, the average size of the F-CIT objects was 228.94 for $t=5$, but 206.63 for $t=6$. We believe that this was because covering a frequently appearing $t$ tuple covers multiple frequently appearing $t^{\prime}$-tuples, where $t^{\prime}<t$. Thus, covering higher strength tuples may help reduce the number of test cases needed by covering more required tuples per test case. Regarding the construction times, except for the experimental setups, in which cutoff $=0$, all the constructions times 
TABLE 18: Statistics about the F-CIT objects created for the $K_{\text {seen }}$ coverage criterion, where the columns, respectively, report the coverage strengths, the frequency cutoff values, the numbers of testable entities to be covered, and the average construction times (in seconds) as well as the average sizes of the F-CIT objects computed by the generate-and-cover and cover-and-generate constructors together with the minimum, maximum, standard deviation, and coefficient of variation statistics for the results obtained from the latter constructor. The character ${ }^{\prime * \prime}$ marks the experimental setups, in which the generate-and-cover constructor timed out after one day. Furthermore, the number of times the experiments were repeated are given in the column "repeat count."

\begin{tabular}{|c|c|c|c|c|c|c|c|c|c|c|c|c|c|c|c|c|}
\hline \multirow[b]{3}{*}{$\mathrm{t}$} & \multirow[b]{3}{*}{ cutoff } & \multirow{3}{*}{$\begin{array}{r}\text { no of } \\
\text { entities }\end{array}$} & \multicolumn{3}{|c|}{$\begin{array}{l}\text { generate-and-cover } \\
\text { constructor }\end{array}$} & \multicolumn{11}{|c|}{ cover-and-generate constructor } \\
\hline & & & avg. & avg. & repeat & & & time & & & & & size & & & repeat \\
\hline & & & time & size & count & $\min$. & avg. & $\max$. & sd. & $\mathrm{cv}$. & $\min$. & avg. & $\max$. & sd. & cv. & count \\
\hline 2 & 0.5 & 4 & 0.02 & 2.00 & 3 & 0.00 & 0.03 & 0.06 & 0.01 & 57.86 & 1 & 1.00 & 1 & 0.00 & 0.00 & 100 \\
\hline 2 & 0.25 & 20 & 0.05 & 8.67 & 3 & 0.01 & 0.08 & 0.19 & 0.05 & 56.35 & 2 & 2.31 & 3 & 0.46 & 20.02 & 100 \\
\hline 2 & 0.2 & 34 & 0.13 & 16.00 & 3 & 0.01 & 0.13 & 0.27 & 0.07 & 56.09 & 3 & 3.89 & 5 & 0.61 & 15.80 & 100 \\
\hline 2 & 0.15 & 54 & 0.25 & 24.00 & 3 & 0.00 & 0.18 & 0.38 & 0.11 & 61.40 & 4 & 4.70 & 7 & 0.62 & 13.29 & 100 \\
\hline 2 & 0.1 & 80 & 0.60 & 38.33 & 3 & 0.02 & 0.29 & 0.60 & 0.16 & 56.09 & 5 & 6.50 & 9 & 0.83 & 12.78 & 100 \\
\hline 2 & 0.05 & 200 & 3.02 & 94.33 & 3 & 0.06 & 0.73 & 1.54 & 0.41 & 55.85 & 13 & 16.00 & 19 & 1.33 & 8.34 & 100 \\
\hline 2 & 0.04 & 240 & 4.51 & 124.00 & 3 & 0.06 & 0.93 & 1.95 & 0.52 & 55.76 & 19 & 21.72 & 25 & 1.46 & 6.74 & 100 \\
\hline 2 & 0.03 & 299 & 6.05 & 152.33 & 3 & 0.04 & 1.14 & 2.40 & 0.66 & 58.07 & 21 & 27.46 & 31 & 1.77 & 6.46 & 100 \\
\hline 2 & 0.02 & 422 & 10.47 & 211.33 & 3 & 0.14 & 1.74 & 3.61 & 0.97 & 55.53 & 35 & 40.53 & 45 & 1.93 & 4.75 & 100 \\
\hline 2 & 0.01 & 669 & 23.79 & 333.00 & 3 & 0.32 & 3.07 & 6.07 & 1.67 & 54.36 & 61 & 69.34 & 75 & 2.82 & 4.06 & 100 \\
\hline 2 & 0.005 & 1056 & 50.97 & 518.33 & 3 & 0.30 & 4.92 & 9.60 & 2.75 & 55.91 & 113 & 118.83 & 126 & 2.91 & 2.44 & 100 \\
\hline 2 & 0.001 & 2652 & 265.32 & 1275.00 & 3 & 2.29 & 14.85 & 27.08 & 7.51 & 50.58 & 311 & 327.31 & 342 & 5.89 & 1.80 & 100 \\
\hline 2 & 0 & 22554 & 10389.32 & 9606.33 & 3 & 228.72 & 364.05 & 493.08 & 75.38 & 20.71 & 3589 & 3625.24 & 3666 & 17.06 & 0.47 & 100 \\
\hline 3 & 0.5 & 4 & 0.01 & 2.33 & 3 & 0.00 & 0.02 & 0.05 & 0.01 & 56.45 & 1 & 1.00 & 1 & 0.00 & 0.00 & 100 \\
\hline 3 & 0.25 & 25 & 0.10 & 8.33 & 3 & 0.01 & 0.08 & 0.18 & 0.04 & 58.47 & 2 & 2.26 & 3 & 0.44 & 19.41 & 100 \\
\hline 3 & 0.2 & 44 & 0.12 & 15.33 & 3 & 0.01 & 0.12 & 0.29 & 0.07 & 58.07 & 3 & 3.82 & 6 & 0.77 & 20.07 & 100 \\
\hline 3 & 0.15 & 89 & 0.61 & 32.00 & 3 & 0.01 & 0.16 & 0.38 & 0.10 & 58.08 & 4 & 4.96 & 8 & 0.95 & 19.11 & 100 \\
\hline 3 & 0.1 & 164 & 1.20 & 53.67 & 3 & 0.03 & 0.28 & 0.68 & 0.17 & 57.97 & 5 & 6.86 & 10 & 1.33 & 19.34 & 100 \\
\hline 3 & 0.05 & 474 & 8.16 & 170.00 & 3 & 0.06 & 0.79 & 2.66 & 0.50 & 63.02 & 14 & 18.54 & 24 & 2.22 & 12.00 & 100 \\
\hline 3 & 0.04 & 601 & 10.22 & 194.00 & 3 & 0.13 & 1.08 & 2.09 & 0.57 & 52.87 & 20 & 26.24 & 32 & 2.32 & 8.84 & 100 \\
\hline 3 & 0.03 & 811 & 20.99 & 285.33 & 3 & 0.12 & 1.37 & 2.82 & 0.74 & 54.17 & 27 & 34.60 & 42 & 3.14 & 9.08 & 100 \\
\hline 3 & 0.02 & 1281 & 40.73 & 456.00 & 3 & 0.19 & 2.30 & 4.48 & 1.26 & 54.66 & 45 & 57.47 & 68 & 3.95 & 6.87 & 100 \\
\hline 3 & 0.01 & 2201 & 106.93 & 781.67 & 3 & 0.58 & 4.66 & 9.26 & 2.48 & 53.10 & 103 & 113.27 & 129 & 4.67 & 4.13 & 100 \\
\hline 3 & 0.005 & 3751 & 251.85 & 1340.67 & 3 & 1.26 & 9.05 & 17.85 & 4.59 & 50.71 & 203 & 217.86 & 238 & 6.87 & 3.15 & 100 \\
\hline 3 & 0.001 & 11604 & 1813.94 & 4137.00 & 3 & 14.21 & 43.31 & 71.85 & 16.66 & 38.46 & 790 & 817.66 & 853 & 12.96 & 1.59 & 100 \\
\hline 3 & 0 & 182952 & 197208.59 & 72642.00 & 2 & 8266.04 & 8266.04 & 8266.04 & $\mathrm{n} / \mathrm{a}$ & $\mathrm{n} / \mathrm{a}$ & 24971 & 24971.00 & 24971 & $\mathrm{n} / \mathrm{a}$ & $\mathrm{n} / \mathrm{a}$ & 1 \\
\hline 4 & 0.5 & 4 & 0.02 & 2.33 & 3 & 0.00 & 0.02 & 0.06 & 0.01 & 60.87 & 1 & 1.00 & 1 & 0.00 & 0.00 & 100 \\
\hline 4 & 0.25 & 26 & 0.05 & 7.67 & 3 & 0.01 & 0.08 & 0.18 & 0.04 & 58.93 & 2 & 2.31 & 3 & 0.46 & 20.02 & 100 \\
\hline 4 & 0.2 & 49 & 0.15 & 16.00 & 3 & 0.01 & 0.11 & 0.28 & 0.07 & 59.04 & 3 & 3.65 & 5 & 0.73 & 19.90 & 100 \\
\hline 4 & 0.15 & 100 & 0.43 & 24.33 & 3 & 0.01 & 0.15 & 0.37 & 0.09 & 57.23 & 4 & 4.91 & 8 & 0.91 & 18.46 & 100 \\
\hline 4 & 0.1 & 235 & 1.69 & 65.33 & 3 & 0.01 & 0.24 & 0.55 & 0.14 & 58.37 & 5 & 6.32 & 10 & 1.22 & 19.23 & 100 \\
\hline 4 & 0.05 & 734 & 10.29 & 188.33 & 3 & 0.07 & 0.66 & 1.29 & 0.34 & 51.88 & 11 & 16.82 & 22 & 2.09 & 12.40 & 100 \\
\hline 4 & 0.04 & 964 & 16.06 & 235.00 & 3 & 0.12 & 0.90 & 1.99 & 0.49 & 54.20 & 16 & 23.26 & 31 & 3.06 & 13.15 & 100 \\
\hline 4 & 0.03 & 1395 & 31.92 & 368.67 & 3 & 0.14 & 1.25 & 2.85 & 0.66 & 52.82 & 26 & 32.07 & 41 & 2.90 & 9.04 & 100 \\
\hline 4 & 0.02 & 2382 & 74.45 & 606.67 & 3 & 0.30 & 2.17 & 4.51 & 1.10 & 50.58 & 44 & 54.46 & 66 & 3.93 & 7.21 & 100 \\
\hline 4 & 0.01 & 4475 & 189.34 & 1136.00 & 3 & 1.10 & 5.07 & 10.46 & 2.43 & 47.96 & 99 & 118.57 & 138 & 7.23 & 6.10 & 100 \\
\hline 4 & 0.005 & 7949 & 503.37 & 2073.33 & 3 & 2.97 & 11.06 & 19.49 & 4.85 & 43.84 & 223 & 248.91 & 270 & 9.51 & 3.82 & 100 \\
\hline 4 & 0.001 & 27890 & 3793.76 & 7266.00 & 3 & 39.78 & 76.39 & 114.12 & 20.34 & 26.63 & 1089 & 1124.70 & 1165 & 17.37 & 1.54 & 100 \\
\hline 4 & 0 & 658825 & * & * & 1 & 62011.15 & 62011.15 & 62011.15 & $\mathrm{n} / \mathrm{a}$ & $\mathrm{n} / \mathrm{a}$ & 59960 & 59960.00 & 59960 & $\mathrm{n} / \mathrm{a}$ & $\mathrm{n} / \mathrm{a}$ & 1 \\
\hline 5 & 0.5 & 4 & 0.01 & 3.00 & 3 & 0.00 & 0.02 & 0.05 & 0.01 & 56.31 & 1 & 1.00 & 1 & 0.00 & 0.00 & 100 \\
\hline 5 & 0.25 & 26 & 0.05 & 8.67 & 3 & 0.01 & 0.08 & 0.19 & 0.05 & 59.27 & 2 & 2.30 & 3 & 0.46 & 19.92 & 100 \\
\hline 5 & 0.2 & 50 & 0.10 & 14.33 & 3 & 0.00 & 0.11 & 0.28 & 0.07 & 60.11 & 3 & 3.76 & 5 & 0.72 & 19.22 & 100 \\
\hline 5 & 0.15 & 101 & 0.39 & 28.33 & 3 & 0.01 & 0.15 & 0.39 & 0.09 & 59.85 & 4 & 4.89 & 7 & 0.86 & 17.57 & 100 \\
\hline 5 & 0.1 & 264 & 1.31 & 53.00 & 3 & 0.02 & 0.21 & 0.46 & 0.12 & 56.31 & 5 & 5.84 & 10 & 1.01 & 17.25 & 100 \\
\hline 5 & 0.05 & 900 & 11.66 & 185.00 & 3 & 0.10 & 0.62 & 1.44 & 0.33 & 52.70 & 13 & 15.91 & 21 & 1.65 & 10.37 & 100 \\
\hline 5 & 0.04 & 1204 & 18.24 & 236.33 & 3 & 0.14 & 0.85 & 1.76 & 0.45 & 52.63 & 16 & 21.74 & 28 & 2.70 & 12.42 & 100 \\
\hline 5 & 0.03 & 1811 & 32.68 & 339.33 & 3 & 0.21 & 1.19 & 4.40 & 0.65 & 54.62 & 24 & 29.79 & 38 & 2.82 & 9.47 & 100 \\
\hline 5 & 0.02 & 3256 & 68.51 & 598.67 & 3 & 0.48 & 2.07 & 4.04 & 0.94 & 45.63 & 40 & 48.82 & 57 & 3.92 & 8.02 & 100 \\
\hline 5 & 0.01 & 6585 & 217.98 & 1188.67 & 3 & 1.20 & 4.64 & 7.92 & 1.95 & 41.96 & 89 & 106.41 & 126 & 7.00 & 6.58 & 100 \\
\hline 5 & 0.005 & 12028 & 564.90 & 2256.67 & 3 & 4.33 & 11.50 & 19.43 & 4.18 & 36.39 & 206 & 228.94 & 244 & 8.89 & 3.88 & 100 \\
\hline 5 & 0.001 & 45599 & 4400.78 & 8645.33 & 3 & 66.72 & 100.02 & 133.16 & 18.82 & 18.81 & 1056 & 1123.06 & 1168 & 20.17 & 1.80 & 100 \\
\hline 5 & 0 & 1240182 & * & * & 1 & 171331.44 & 171331.44 & 171331.44 & $\mathrm{n} / \mathrm{a}$ & $\mathrm{n} / \mathrm{a}$ & 88314 & 88314.00 & 88314 & $\mathrm{n} / \mathrm{a}$ & $\mathrm{n} / \mathrm{a}$ & 1 \\
\hline 6 & 0.5 & 4 & 0.01 & 2.33 & 3 & 0.00 & 0.03 & 0.05 & 0.01 & 56.15 & 1 & 1.00 & 1 & 0.00 & 0.00 & 100 \\
\hline 6 & 0.25 & 26 & 0.03 & 5.00 & 3 & 0.01 & 0.07 & 0.18 & 0.04 & 56.12 & 2 & 2.22 & 3 & 0.41 & 18.66 & 100 \\
\hline 6 & 0.2 & 50 & 0.14 & 14.33 & 3 & 0.00 & 0.11 & 0.64 & 0.08 & 75.56 & 3 & 3.73 & 5 & 0.72 & 19.28 & 100 \\
\hline 6 & 0.15 & 101 & 0.36 & 27.00 & 3 & 0.01 & 0.15 & 0.41 & 0.09 & 57.95 & 4 & 4.73 & 7 & 0.86 & 18.15 & 100 \\
\hline 6 & 0.1 & 269 & 1.18 & 43.33 & 3 & 0.02 & 0.20 & 0.59 & 0.12 & 57.36 & 5 & 5.84 & 10 & 0.97 & 16.55 & 100 \\
\hline 6 & 0.05 & 971 & 10.15 & 155.67 & 3 & 0.07 & 0.57 & 1.27 & 0.30 & 52.93 & 13 & 15.45 & 20 & 1.66 & 10.77 & 100 \\
\hline 6 & 0.04 & 1318 & 16.74 & 223.33 & 3 & 0.11 & 0.80 & 1.64 & 0.40 & 50.33 & 17 & 21.15 & 29 & 2.22 & 10.47 & 100 \\
\hline 6 & 0.03 & 2001 & 25.10 & 280.67 & 3 & 0.24 & 1.14 & 2.17 & 0.53 & 46.50 & 24 & 28.33 & 35 & 2.45 & 8.63 & 100 \\
\hline 6 & 0.02 & 3705 & 68.32 & 538.33 & 3 & 0.51 & 1.93 & 3.87 & 0.84 & 43.62 & 38 & 46.23 & 60 & 3.58 & 7.74 & 100 \\
\hline 6 & 0.01 & 7825 & 196.90 & 1061.00 & 3 & 1.39 & 4.44 & 8.35 & 1.77 & 39.80 & 78 & 95.96 & 112 & 6.31 & 6.58 & 100 \\
\hline 6 & 0.005 & 14634 & 493.02 & 2031.00 & 3 & 5.10 & 10.83 & 17.33 & 3.52 & 32.50 & 186 & 206.63 & 225 & 7.91 & 3.83 & 100 \\
\hline 6 & 0.001 & 57741 & 3880.13 & 7842.67 & 3 & 74.39 & 105.56 & 139.61 & 16.58 & 15.71 & 963 & 1015.85 & 1065 & 20.88 & 2.06 & 100 \\
\hline 6 & 0 & 1321685 & . & * & 1 & 179456.00 & 179456.00 & 179456.00 & $\mathrm{n} / \mathrm{a}$ & $\mathrm{n} / \mathrm{a}$ & 80350 & 80350.00 & 80350 & $\mathrm{n} / \mathrm{a}$ & $\mathrm{n} / \mathrm{a}$ & 1 \\
\hline
\end{tabular}


TABLE 19: Statistics about the F-CIT objects created for the $K_{\text {weighted }}$ coverage criterion, where the columns, respectively, report the coverage strengths, the weight cutoff values, the numbers of testable entities to be covered, and the average construction times (in seconds) as well as the average sizes of the F-CIT objects computed by the generate-and-cover and cover-and-generate constructors together with the minimum, maximum, standard deviation, and coefficient of variation statistics for the results obtained from the latter constructor. Furthermore, the number of times the experiments were repeated are given in the column "repeat count."

\begin{tabular}{|c|c|c|c|c|c|c|c|c|c|c|c|c|c|c|c|c|}
\hline \multirow[b]{3}{*}{$\mathrm{t}$} & \multirow[b]{3}{*}{ cutoff } & \multirow{3}{*}{$\begin{array}{r}\text { no of } \\
\text { entities }\end{array}$} & \multicolumn{3}{|c|}{$\begin{array}{l}\text { generate-and-cover } \\
\text { constructor }\end{array}$} & \multicolumn{11}{|c|}{ cover-and-generate constructor } \\
\hline & & & avg. & avg. & repeat & & & time & & & & & size & & & repeat \\
\hline & & & time & size & count & $\min$. & avg. & $\max$. & sd. & $\mathrm{cv}$. & $\min$. & avg. & $\max$ & sd. & $\mathrm{cv}$. & count \\
\hline 2 & 0.70 & 332 & 7.16 & 167.67 & 3 & 0.07 & 0.76 & 1.73 & 0.48 & 62.75 & 27 & 31.74 & 37 & 1.97 & 6.22 & 100 \\
\hline 2 & 0.75 & 426 & 10.23 & 209.67 & 3 & 0.09 & 0.99 & 2.16 & 0.63 & 63.43 & 36 & 41.10 & 47 & 2.20 & 5.36 & 100 \\
\hline 2 & 0.80 & 566 & 17.90 & 279.33 & 3 & 0.13 & 1.38 & 3.03 & 0.88 & 63.99 & 49 & 55.73 & 61 & 2.26 & 4.05 & 100 \\
\hline 2 & 0.85 & 793 & 34.33 & 400.33 & 3 & 0.24 & 2.11 & 4.64 & 1.30 & 61.60 & 77 & 85.40 & 94 & 3.00 & 3.51 & 100 \\
\hline 2 & 0.90 & 1185 & 63.95 & 574.67 & 3 & 0.46 & 3.33 & 7.03 & 1.99 & 59.87 & 126 & 133.28 & 141 & 3.60 & 2.70 & 100 \\
\hline 2 & 0.95 & 2073 & 162.53 & 990.33 & 3 & 1.10 & 6.42 & 14.11 & 3.73 & 58.04 & 235 & 245.66 & 256 & 4.46 & 1.82 & 100 \\
\hline 3 & 0.70 & 2171 & 100.70 & 777.33 & 3 & 0.49 & 2.73 & 5.86 & 1.54 & 56.41 & 100 & 111.32 & 120 & 4.15 & 3.73 & 100 \\
\hline 3 & 0.75 & 2951 & 181.72 & 1090.00 & 3 & 0.89 & 4.14 & 8.51 & 2.25 & 54.34 & 150 & 164.80 & 178 & 5.04 & 3.06 & 100 \\
\hline 3 & 0.80 & 4117 & 303.40 & 1488.67 & 3 & 1.59 & 6.60 & 12.70 & 3.40 & 51.50 & 227 & 242.96 & 263 & 6.28 & 2.58 & 100 \\
\hline 3 & 0.85 & 6051 & 582.78 & 2164.00 & 3 & 3.40 & 11.20 & 21.29 & 5.25 & 46.83 & 362 & 384.02 & 405 & 8.09 & 2.11 & 100 \\
\hline 3 & 0.90 & 9675 & 1291.66 & 3447.33 & 3 & 9.26 & 22.47 & 38.32 & 8.79 & 39.12 & 639 & 666.56 & 696 & 10.16 & 1.52 & 100 \\
\hline 3 & 0.95 & 17826 & 3671.09 & 6332.00 & 3 & 34.85 & 63.21 & 94.30 & 17.61 & 27.86 & 1320 & 1347.32 & 1387 & 14.03 & 1.04 & 100 \\
\hline 4 & 0.70 & 8037 & 493.52 & 2095.67 & 3 & 3.07 & 7.74 & 13.70 & 3.07 & 39.64 & 218 & 251.25 & 275 & 8.98 & 3.57 & 100 \\
\hline 4 & 0.75 & 11240 & 850.59 & 2905.67 & 3 & 6.23 & 13.25 & 25.50 & 4.72 & 35.59 & 357 & 382.40 & 414 & 10.73 & 2.80 & 100 \\
\hline 4 & 0.80 & 16252 & 1608.39 & 4222.33 & 3 & 12.85 & 23.91 & 37.19 & 7.35 & 30.76 & 568 & 605.22 & 645 & 13.96 & 2.31 & 100 \\
\hline 4 & 0.85 & 24798 & 3177.50 & 6454.67 & 3 & 31.82 & 50.10 & 71.79 & 11.96 & 23.88 & 947 & 984.22 & 1039 & 19.26 & 1.96 & 100 \\
\hline 4 & 0.90 & 40330 & 6942.08 & 10454.67 & 3 & 90.62 & 127.55 & 200.33 & 23.77 & 18.64 & 1675 & 1734.26 & 1816 & 24.83 & 1.43 & 100 \\
\hline 4 & 0.95 & 79330 & 21111.57 & 20702.00 & 3 & 403.89 & 459.80 & 500.68 & 19.50 & 4.24 & 3768 & 3868.14 & 3923 & 29.77 & 0.77 & 100 \\
\hline 5 & 0.70 & 18518 & 1070.09 & 3516.67 & 3 & 10.01 & 16.76 & 24.58 & 4.39 & 26.17 & 362 & 386.81 & 413 & 11.02 & 2.85 & 100 \\
\hline 5 & 0.75 & 26467 & 1896.90 & 5018.33 & 3 & 20.37 & 31.69 & 46.81 & 7.37 & 23.24 & 573 & 608.41 & 662 & 15.30 & 2.51 & 100 \\
\hline 5 & 0.80 & 39309 & 3540.80 & 7484.33 & 3 & 47.71 & 66.26 & 109.47 & 12.47 & 18.83 & 911 & 956.32 & 1003 & 19.17 & 2.00 & 100 \\
\hline 5 & 0.85 & 60222 & 6776.82 & 11397.67 & 3 & 115.87 & 132.83 & 148.14 & 7.46 & 5.61 & 1499 & 1555.03 & 1612 & 25.24 & 1.62 & 100 \\
\hline 5 & 0.90 & 101015 & 15938.67 & 19338.33 & 3 & 362.41 & 411.02 & 439.87 & 14.26 & 3.47 & 2751 & 2849.14 & 2937 & 37.23 & 1.31 & 100 \\
\hline 5 & 0.95 & 211007 & 50420.67 & 40552.50 & 2 & 1977.17 & 2081.92 & 2156.12 & 39.50 & 1.90 & 6738 & 6857.15 & 6974 & 48.97 & 0.71 & 100 \\
\hline 6 & 0.70 & 29272 & 1380.18 & 4027.33 & 3 & 18.73 & 27.23 & 40.74 & 5.67 & 20.81 & 442 & 475.20 & 502 & 12.64 & 2.66 & 100 \\
\hline 6 & 0.75 & 42694 & 2511.12 & 5857.00 & 3 & 40.92 & 54.86 & 90.64 & 9.36 & 17.06 & 708 & 743.64 & 789 & 16.65 & 2.24 & 100 \\
\hline 6 & 0.80 & 63498 & 4650.73 & 8831.33 & 3 & 86.44 & 102.17 & 113.87 & 5.70 & 5.58 & 1065 & 1113.34 & 1156 & 20.65 & 1.85 & 100 \\
\hline 6 & 0.85 & 98886 & 9152.57 & 13601.33 & 3 & 227.07 & 260.64 & 288.15 & 10.86 & 4.17 & 1765 & 1853.89 & 1936 & 32.88 & 1.77 & 100 \\
\hline 6 & 0.90 & 169254 & 22546.59 & 23611.67 & 3 & 775.99 & 846.07 & 896.63 & 25.20 & 2.98 & 3359 & 3449.67 & 3540 & 37.66 & 1.09 & 100 \\
\hline 6 & 0.95 & 368650 & 75936.20 & 52238.00 & 1 & 4482.59 & 4611.18 & 4785.40 & 61.42 & 1.33 & 8495 & 8627.77 & 8794 & 57.54 & 0.67 & 100 \\
\hline
\end{tabular}

were under 106 seconds, with a majority of them being under 12 seconds, on average.

Evaluating the $K_{\text {weighted }}$ coverage criterion. Table 19 summarizes the results we obtained from the experiments, in which we used the $K_{\text {weighted }}$ coverage criterion.

As was the case with $K_{\text {seen }}$, the cover-and-generate constructor, compared to the generate-and-cover constructor, computed smaller covering arrays at a fraction of the cost. More specifically, the cover-and-generate constructor reduced the sizes by an average of $77.39 \%$, $80.93 \%, 83.29 \%, 83.09 \%$, and $80.29 \%$ while at the same time reducing the construction times by an average of $94.94 \%, 98.20 \%, 98.00 \%, 95.88 \%$, and $92.00 \%$, when $t=2,3,4,5$, and 6 , respectively. We, therefore, focus on the results obtained from the cover-and-generate constructor in the remainder of this section.

We observed that the sizes of all the F-CIT objects we computed for the study, were profoundly smaller than the number of distinct configurations observed in the field (Table 19). More specifically, the maximum average size was 8627.77 , which occurred when $t=6$ and the weighted cutoff was 0.95 . That is, to cover $95 \%$ of the most frequently appearing $t$-tuples for all $1 \leq t \leq 6$ in a weighted manner as described in Definition 28, an F-CIT object of size 8627.77 was needed, on average.

For a fixed strength, as the cutoff decreased, the number of testable entities to be covered as well as the size of the F-CIT objects tended to decrease. For example, when $t=6$, the sizes of the F-CIT objects for cutoff $=0.95,0.90,0.85,0.80,0.75$, and 0.70 , were, respectively, 8627.77, 3449.67, 1853.89, 1113.34, 743.64, and 475.20 (Table 19). Similarly, for a fixed cutoff, as the coverage strength decreased, both the number of testable entities to be covered as well as the size of the F-CIT objects tended to decrease. For example, when cutoff $=0.95$, the average sizes were $8627.77,6857.15$, $3868.14,1347.32$, and 245.66 for $t=6,5,4,3$, and 2 , respectively. Last but not least, the maximum average construction time in all the experiments was 4611.18 seconds, which happened when $t=6$ and cutoff $=0.95$. A majority of the construction times $(79.4 \%)$ were, on the other hand, under 150 seconds (Table 19).

Evaluating sensitivity to the order of processing. Tables 18 and 19 report the minimum, maximum, standard deviation, and coefficient of variation (i.e., the ratio of the standard variation to the mean, in short 
$\mathrm{CV})$ results obtained from the cover-and-generate constructor by repeating the experiments 100 times (except for the experimental setups where the frequency cutoff was 0 and $t>2$, which were repeated only once due to their costs). Clearly, the performance of the cover-andgenerate constructor can be affected by the order, in which the testable entities are processed. Consequently, in the absence of any knowledge regarding a favorable order (or a partial order), a random order can be used by shuffling the entities to be covered before they are fed to the constructor. This process can further be repeated multiple times in an attempt to generate smaller CIT objects at the cost of increased construction times.

\subsubsection{Discussion}

Note that the maximum coverage strength that can be used with the $K_{\text {weighted }}$ coverage criterion is the number of configuration options that the system under test has. Therefore, $K_{\text {weighted }}$, in a sense, offers a solution to an important, but still an open question of how to determine the coverage strength in CIT, by automatically determining strength based on usage statistics. That is, the strength of a tuple to be covered by $K_{\text {weighted }}$, essentially depends on how frequently the tuple appears in the field. Consequently, the strength may vary across the test space. This is different than variable strength covering arrays [56], because in variable strength covering arrays, the strengths are determined a priori and they vary at the level of option combinations. In the $K_{\text {weighted }}$ coverage criterion, on the other hand, the strengths vary at the level of option setting combinations and they are determined based on usage statistics. Therefore, no strength needs to be determined beforehand.

Note that F-CIT does not aim to replace standard covering array constructors. We, indeed, don't see much value in using F-CIT to compute the same CIT objects that the existing CIT constructors compute, as the generalized F-CIT constructors may not be as efficient and as effective as their specialized counterparts. For example, when we used the cover-and-generate constructor to compute standard covering arrays for the configuration space models used in the experiments, the aforementioned F-CIT constructor generated a 2-way standard covering array of size 87586 in 32263.90 seconds (vs. a 2-way covering array of size 86241 generated in 100 seconds by ACTS) and failed to generate a 3-way standard covering array within a day, after which we stopped the constructor (vs. a 3way covering array of size 13283730 generated in 32 minutes by ACTS).

The point we want to emphasize, however, is that even if F-CIT was able to reduce the sizes by half and did so in seconds, it would still not be feasible at all (for the consumer company, for which we carried out the study) to run all the test cases selected. Therefore, the coverage criteria needed to be changed. However, existing constructors, as they are, could not take advantage of these new criteria, which required fewer tuples to be covered.

Last but not least, it seems that for the usage-based CIT problem, it may actually be possible to modify an existing constructor. This, however, requires that the source code of the constructor is available, the code is reversed engineered, and a modification strategy is implemented, tested, and maintained. Note, however, that even if this was possible, these modifications would be of little help (or of no help at all) to compute the structure-based and order-based CIT objects we discussed in Section 5.1 and Section 5.2, respectively. Consequently, another set of modifications would be required to compute the structure-based CIT objects, such that the values of model parameters can be expressed as arbitrarily complex Boolean expressions. Similarly, different set of modifications would be required to compute the order-based CIT objects, such that the reachability restrictions imposed by a graphbased model can be expressed as constraints to cover various orders of nodes. As a matter of fact, we don't know how these modifications can be made without the solution quickly converging to F-CIT. This is exactly why F-CIT aims to eliminate the need of modifying existing constructors or developing specialized constructors, by generalizing the construction of CIT objects as much as possible.

\section{User Studies}

To further evaluate the proposed approach, we have also carried out user studies.

\subsection{Study Setup}

We asked the Junior, Senior, and graduate-level computer science students studying at Sabanci University whether they would take part in the study. A total of 13 graduate-level and 7 undergraduate-level students agreed to participate on a voluntary basis. Table 20 summarizes the demographic information about the participants. Note that students at Sabanci University study standard combinatorial interaction approaches at different levels and/or for different purposes in the Software Engineering (undergraduate level), Software Verification and Validation (undergraduate/graduate level), and Automated Debugging (graduate level) courses, which explains the participants knowledgable of CIT in Table 20.

The participants were first given a 1-hour lecture. In this lecture, after a brief introduction of how the study would be carried out, the basic concepts in constraint solving, such as Boolean logic and satisfiability, were discussed. Then, F-CIT was introduced. To this end, 
TABLE 20: Demographic information about the participants.

\begin{tabular}{|c|c|c|c|c|c|c|c|c|}
\hline & \multicolumn{4}{|c|}{ undergraduate } & \multicolumn{4}{|c|}{ graduate } \\
\hline no of participants & \multicolumn{4}{|c|}{7} & \multicolumn{4}{|c|}{13} \\
\hline \multirow{2}{*}{ knowledgeable of CIT } & \multirow{2}{*}{\multicolumn{2}{|c|}{ yes }} & \multirow{2}{*}{\multicolumn{2}{|c|}{$\begin{array}{c}\text { no } \\
6\end{array}$}} & \multirow{2}{*}{\multicolumn{2}{|c|}{$\begin{array}{c}\text { yes } \\
10\end{array}$}} & \multicolumn{2}{|c|}{ no } \\
\hline & & 1 & & & & 10 & \multicolumn{2}{|c|}{3} \\
\hline \multirow{2}{*}{$\begin{array}{c}\text { experience in } \\
\text { programming (in years) }\end{array}$} & $\leq 2$ & 3 & 4 & $\geq 5$ & $\leq 2$ & 3 & 4 & $\geq 5$ \\
\hline & 2 & 3 & 2 & 0 & 0 & 2 & 5 & 6 \\
\hline \multirow{2}{*}{$\begin{array}{l}\text { experience in software } \\
\text { testing (in years) }\end{array}$} & $\leq 2$ & 3 & 4 & $\geq 5$ & $\leq 2$ & 3 & 4 & $\geq 5$ \\
\hline & 7 & 0 & 0 & 0 & 11 & 1 & 0 & 1 \\
\hline
\end{tabular}

the definitions and the algorithms given in Sections 34 were studied. Finally, a short tutorial on the F-CIT tool, which we had developed for the study was given (see below for more information about this tool).

The participants, after taking the lecture, took part in the study at their spare times. To gain better insight, each participant carried out the study with one of the authors playing the role of an observer, sitting by the participant and taking notes. The participants were asked to think out loud as much as possible. When it was not clear for the observer what the participant was doing, the observer prompted the participant with questions, such as what do you want to do now? Is the output what you were expecting? What do you think what went wrong? etc.

Each participant was given with the same three problems. These problems were, indeed, the smaller instances of the very same problems we studied in Section 5.1, Section 5.2, and Section 5.3, respectively. For each problem, the participants were first asked to develop an F-CIT model $M=\langle P, D, C\rangle$, then to express a number of F-CIT entities as constraints using $M$, and finally to generate an F-CIT object (by using the F-CIT constructor provided) to cover all of the given entities. The problems as well as the entities used in the study were given in Table 21.

Note that F-CIT is not a methodology for choosing the entities to be tested. It rather takes as input a set of entities to be tested. Therefore, the participants in the study were given with a set of entities to cover. For each problem, the entities were presented starting from the easier ones progressing to the more challenging ones. Furthermore, the number of entities was kept small not to tire the participants.

We designed the studies such that if a participant working on a problem got stuck after the first 10 minutes, the observer would remind the participant of the basic concepts that 1) the F-CIT model $M=<P, D, C>$ should define a set of parameters $P$ and their domains $D ; 2$ ) the model constraint $C$ is a constraint that should be satisfied by all of the FCIT test cases generated; and 3) the entities should be expressed as constraints over $P$.

Furthermore, for the second problem (Table 21), if the participant got stuck after the first 15 minutes, he/she was provided with a description of the network
TABLE 21: Problems used in the user studies.

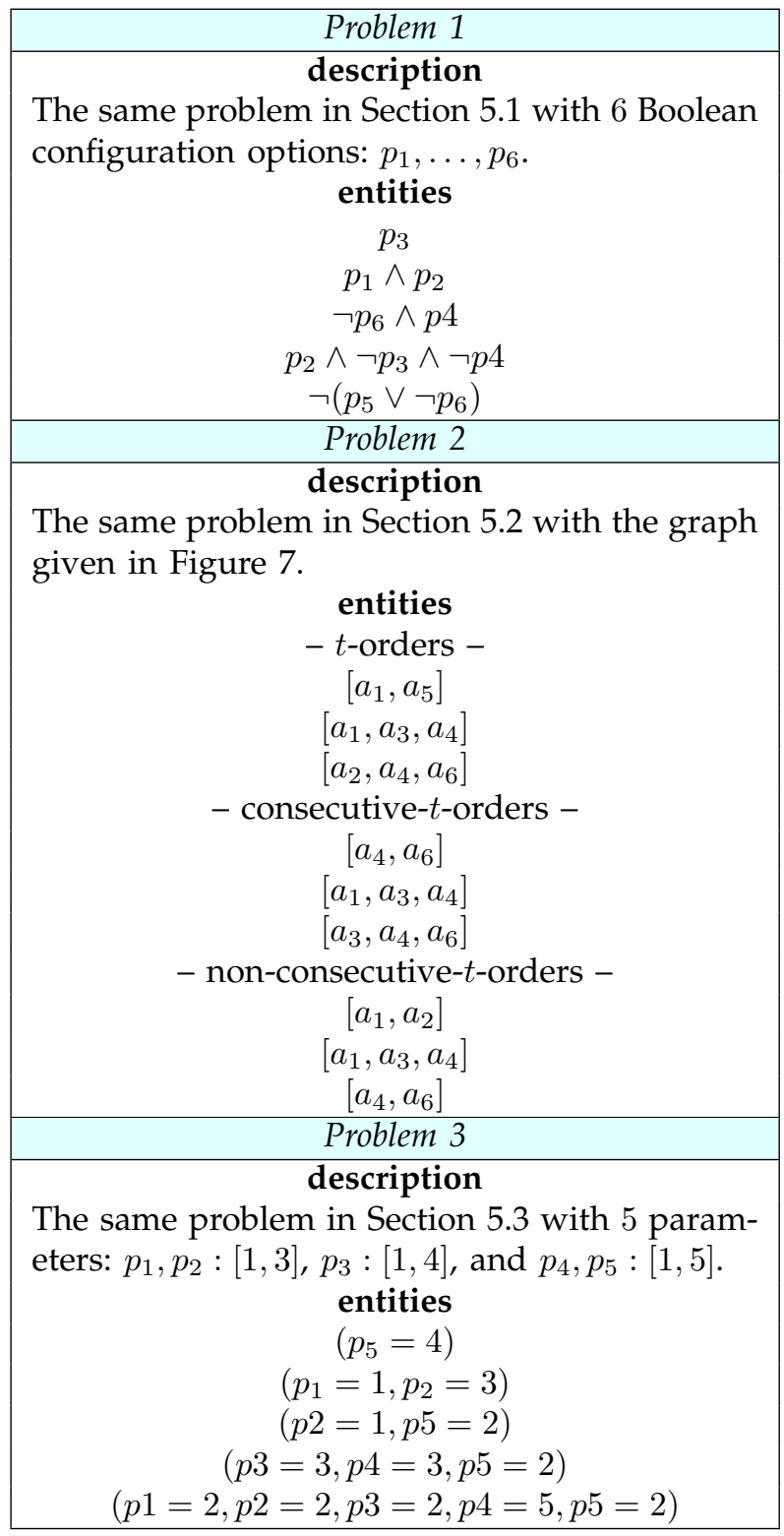

flow problem [57] given in Figure 6a. If the participant got stuck again 15 minutes after reading the description, a solution for the example flow problem given in Figure $6 \mathrm{~b}$ was presented to the participant. Note that both the description in Figure 6a and the example in Figure $6 \mathrm{~b}$ are general enough that they can be found in 
- The graph has a source vertex ' $s$ ' and a terminating vertex ' $t$ '.

- Every edge 'e' has a capacity.

- No edge can have flow exceeding its capacity.

- For every vertex, except for ' $s$ ' and ' $t$ ', the amount of total incoming flow to the vertex must be the same as the amount of total outgoing flow.

- To visit a vertex, there must be an incoming flow to the vertex, i.e., there must be flow on at least one of the incoming edges to the vertex.

(a)

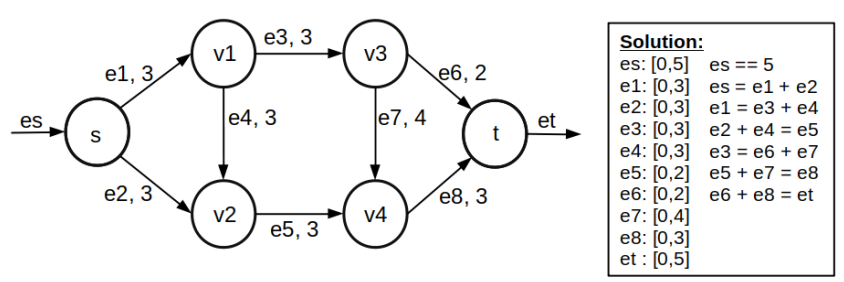

(b)

Fig. 6: Explanations used for the second problem in the user study: (a) the description of the network flow problem and (b) an example network flow with incoming flow as 5 (i.e., es $=5$ ) and its solution, where each

edge has a label in the form of $e x, c$, indicating that $c$ (except $e s$ and $e t$ ) is the capacity of the edge $e x$.

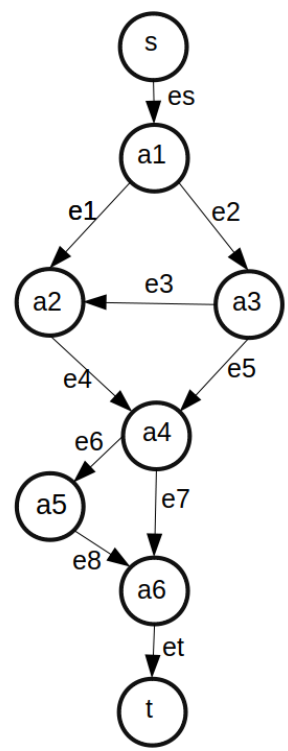

Fig. 7: The graph-based model used in the user studies.

any textbook on the subject [57]. Given these artifacts, the participants were still required to figure out how to express reachability in a graph as a flow problem and how to express different types of order-based entities (Section 5.1) as flow constraints.

We did this because solving the aforementioned problem requires specific knowledge of network flow problems and not all participants might have had the right background. Therefore, by providing a general description of the network flow problems together with an example, we aim to answer the following question: Had the participants had a basic background information on network flow problems, could they have leveraged it in F-CIT to obtain full coverage under various order-based coverage criteria?

Last but not least, we have developed an F-CIT tool for the practitioners to use in the study. Figure 8 presents a screen dump taken from this tool. At a very high level, the tool has three frames. A description frame on the left, presenting the problem to be solved. An F-CIT frame in the middle where the participant
TABLE 22: The exit survey used in the user studies.

All the questions, except for the last two, were Likert scale questions. Questions 1-2 and 6-8 had the following answer options: 1 - strongly disagree, 2 disagree, 3 - neutral, 4 - agree, and 5 - strongly agree. And, questions 3-5 had the following answer options: 1 - very difficult, 2 - difficult, 3 - normal, 4 - easy, and 5 - very easy. The last three questions (5-7) were open-ended questions.

\begin{tabular}{|r|l|}
\hline no & question \\
Q1 & I understand the following concepts: \\
b & constraints \\
c & satisfiability \\
\hline Q2 & I undisfiability \\
a & F-CIT models \\
b & F-CIT entities \\
c & F-CIT test cases \\
d & F-CIT objects \\
\hline Q3 & For problem 1 - Difficulty of encoding: \\
a & F-CIT model \\
b & F-CIT entities \\
\hline Q4 & For problem 2 - Difficulty of encoding: \\
a & F-CIT model \\
b & F-CIT entities \\
\hline Q5 & For problem 3 - Difficulty of encoding: \\
a & F-CIT model \\
b & F-CIT entities \\
\hline Q6 & I found F-CIT useful. \\
\hline Q7 & I would use F-CIT in projects. \\
\hline Q8 & I would recommend F-CIT to others. \\
\hline Q9 & What was the most challenging part in \\
& the study? \\
\hline Q10 & Any suggestions to improve F-CIT? \\
\hline
\end{tabular}

expresses a solution to the given problem in F-CIT. An output frame on the right, which (among other things, see below for more information) presents the results obtained from the cover-and-generate constructor (Section 4.1) for the F-CIT formulation given in the middle frame. 


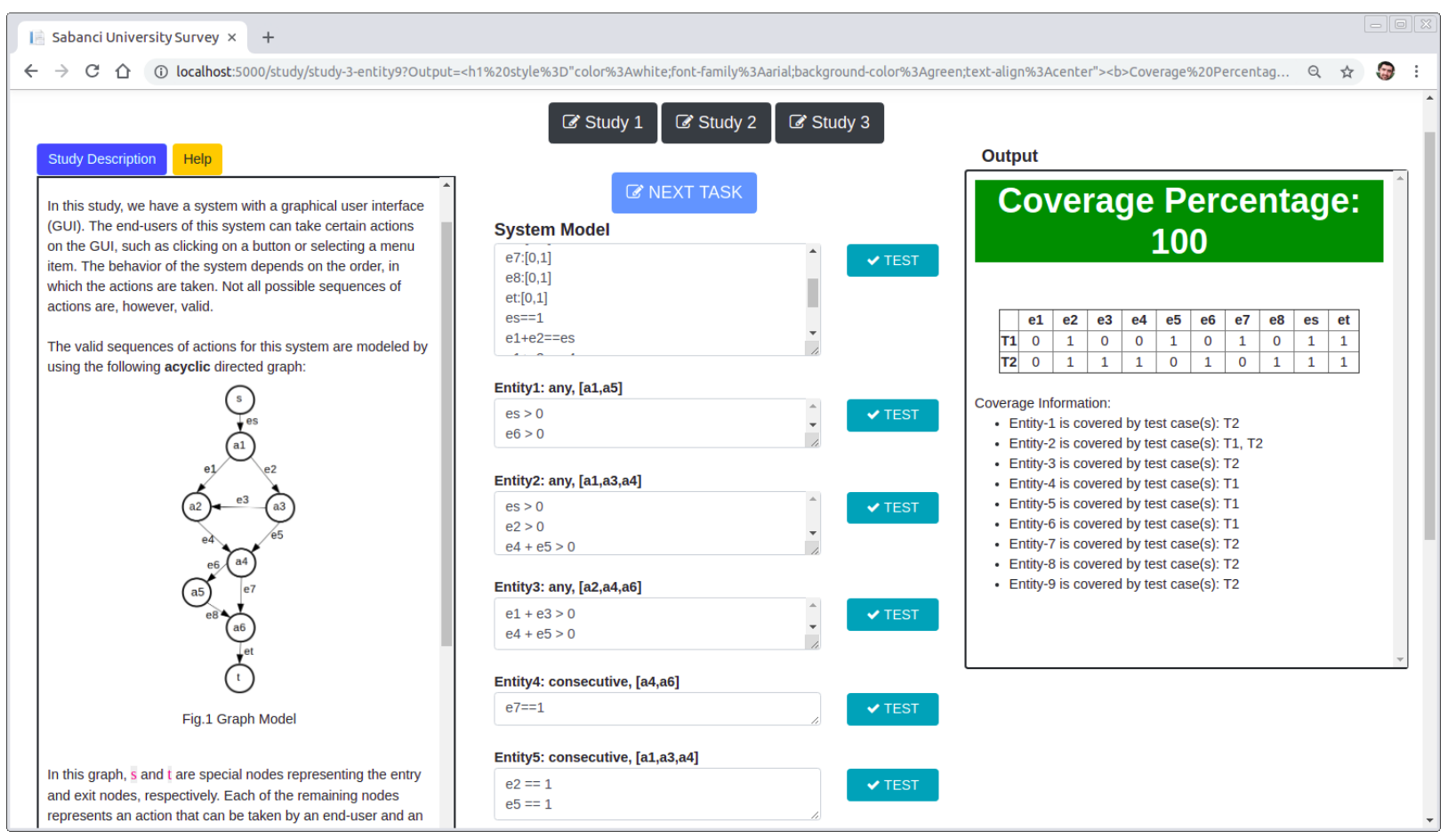

Fig. 8: A screen dump of the tool we have developed for the user studies.

The middle frame had a multi-line text field (model field) to express the F-CIT model and a multi-line text field (entity field) for expressing each entity to be covered. Each field had a "Test" button associated with it. When the Test button of the model field was clicked, the constraints entered for this field were fed to a constraint solver and the result was displayed in the output frame, allowing the participant to check whether the F-CIT model is capable of generating valid test cases. When the Test button of an entity field was clicked, on the other hand, the constraints entered in the respective entity field and those in the model field were combined and fed to the constraint solver. The result was then displayed in the output frame, allowing the participant to check whether the entity can be covered in a valid test case. In both cases, when the constraints were satisfiable, the output frame presented a solution where each parameter defined in the F-CIT model took on a valid value. Otherwise, a warning message indicating that the constraints were not satisfiable, was emitted.

In addition to the Test buttons, we also had a "Generate" button, which fed all the constraints entered (the ones entered in the model and entity fields) to the cover-and-generate constructor (Section 4.1) to compute an F-CIT object. When an F-CIT object was created (which is, indeed, a set of test cases), it was displayed in the output frame in the form of a table, where rows represented the test cases generated and columns depicted the parameters defined in the F-CIT model (Figure 8). Furthermore, the entities covered by each test case are reported.

After completing all the studies, participants filled out an exit survey. Table 22 presents this survey.

\subsection{Evaluation Framework}

To evaluate the proposed approach, we first counted the number of successful formulations. For a given problem, we define a successful formulation as a formulation where both the F-CIT model and the entities to be covered are correctly expressed in a generalizable manner, such that an F-CIT object obtaining full coverage can be computed. Note that we also take the generalizability of the formulation into account because we observed that (solely for the second problem) some participants came up with formulations that are too specific for the problem instance given in the study and that, therefore, are non-trivial to generalize for other instances of the same problem. These formulations were often obtained by introducing additional constraints in the F-CIT model in an ad hoc manner just to avoid some undesirable results. More discussion on this can be found in Section 6.3.

We also measured the time it took for the participants to complete the study. More specifically, for a given problem, we measured the completion time as the difference between the time the description of the problem was presented to the participant and the time the participant completed the study. Note that a study was completed whenever the participant chose to finish the study. In all but two cases, this 
TABLE 23: Demographic information about the participants categorized based on their performances in addressing the second problem.

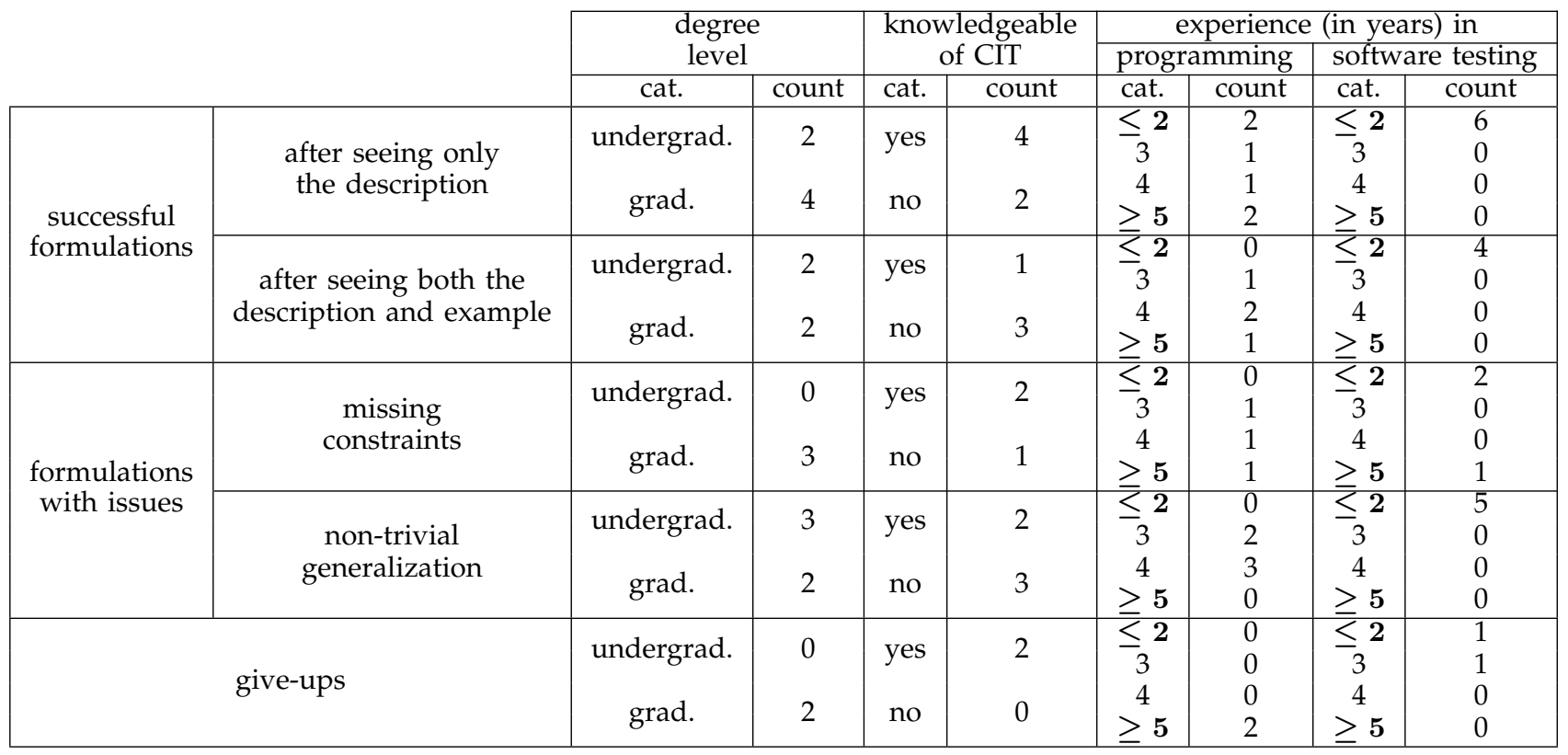

happened after computing an F-CIT object achieving full coverage. In two cases, however, the participants chose to stop working on the current problem in the middle of the study as they found the problem "very difficult" (see Section 6.3 for more information). Each participant worked on the problems one after another.

We, furthermore, counted the number of errors made by the participants. To this end, we counted the number of times the Test and the Generate buttons were clicked (Section 6.1) and the result obtained did not meet the expectation of the participant. More specifically, if a participant, after clicking on a Test button or a Generate button, made some changes and clicked on the same button, we assumed that the participant made an error before the first click (as the expectation of the participant after the first click did not seem to be met). Note that this metric provides an approximation of the number of errors made because on numerous occasions, we observed that the participants intentionally developed incorrect or missing constraints to test their hypotheses or to gain insight into the problem. We still opted to use this metric because attempting to figure out the actual intention of the participant after every click of a button would have introduced a great deal of intervention.

Furthermore, the percentage of the participants, who "agreed" or "strongly agreed" with a question group in the exit survey, was computed as the average percentage of the participants, who "agreed" or "strongly agreed" with the questions in the group. The percentage of the participants, who found the problems "difficult" or "very difficult," is computed in the same manner.

\subsection{Data and Analysis}

We first observed that all of the participants understood how F-CIT works. In particular, none of the participants were reminded of the basic F-CIT concepts during the study.

We then observed that the participants could also formulate previously unseen problems in F-CIT. More specifically, all of the participants successfully formulated the first and the third problems. That is, for each of these problems, all of the participants correctly expressed the F-CIT model as well as all of the F-CIT entities to be covered in a generalizable manner and generated an F-CIT object obtaining full coverage. And, they did so in a relatively fast manner. The average time it took for the participants to complete these studies was 4.88 minutes $(\min =1.47$ and $\max =13.89)$ for the first problem and 5.38 minutes $(\min =2.29$ and $\max =9.02)$ for the third problem.

As expected, the participants found the second problem more difficult than the other two problems, which was also reflected on the outcomes of the exit survey. While $65 \%$ of the participants found the second problem "difficult" or "very difficult," none of the participants thought the same thing for the first and third problems. As a matter of the fact, based on the answers given to the open-ended Q9 (Table 22), the most challenging part in the entire study turned out to be expressing an F-CIT model for the second problem. Two participants, indeed, chose to terminate this study in the middle of it after spending 42.56 minutes on average as they found the problem "very difficult" (see the row marked with "give-ups" in Table 23 for the demographic information of these participants). 
Half (10 out of 20) of the participants, however, successfully formulated the problem in a generalizable manner by using the same (or similar) approach introduced in Section 5.2.4 and obtained full coverage. 6 of them did so after seeing the description in Figure 6a and 4 after seeing both the description and the example flow problem in Figure $6 \mathrm{~b}$ (see the rows marked with "after seeing only the description" and "after seeing both the description and example" in Table 23, respectively, for the demographic information of these participants). None of the participants, who came up with a generalizable solution for this problem, did so without seeing the description or the example. The average completion time was 47.14 minutes $(\min =25.79$ and $\max =69.49$ ).

The remaining $40 \%$ (8 out of 20 ) of the participants, although generated F-CIT objects obtaining full coverage, either came up with a formulation, the generalization of which was non-trivial, or covered some of the entities by chance. More specifically, 3 participants developed generalizable formulations by representing edges using Boolean variables (rather than using integer variables), which were quite similar to the formulation we developed in Section 5.2.4. However, the constraints, which should have invalidated the presence of multiple independent flows, were missing from the F-CIT models (see the row marked with "missing constraints" in Table 23 for the demographic information of these participants). The participants failed to identify the issue because their formulations happened to obtain full coverage by generating valid test cases for the graph given in the study. Had they worked on larger graphs, however, they might have pinpointed and fixed the issue. The average completion time for this category of participants was 37.36 minutes $(\min =33.91$ and $\max =43.01)$.

The remaining 5 participants developed formulations, the generalizations of which were non-trivial (see the row marked with "non-trivial generalization" in Table 23 for the demographic information of these participants). In particular, all of these participants chose to represent each vertex (rather than each edge) in the graph by using an integer variable, the value of which represents the order in which the vertex is visited. For a given variable, the set of possible values were determined manually by considering all possible paths that could be traversed. The invalid combinations of variable values (i.e., invalid paths) were then prevented by introducing model constraints in a rather ad hoc manner every time the participant observed that some of the generated test cases were invalid and/or some of the entities could not be covered. 4 (out of 5) of these participants correctly expressed all the constraints as well as the entities for the graph given in the study and obtained full coverage. The remaining participant, although had some missing and/or faulty constraints in the F-CIT model, happened to obtain full coverage by chance. The average completion time for this category of participants was 46.42 minutes $(\min =23.26$ and $\max =64.54)$.

We did not find any correlations between the performances of the participants and their levels of degree, knowledge of CIT, or experiences in programming and testing. We, however, observed that the knowledge of the domain was influential in successfully completing the studies. More specifically, for the first and third problems, which required basic knowledge of programming and testing, all of the participants successfully formulated the problems in F-CIT. For the second problem, which required basic knowledge of network flow problems, all of the participants, who successfully formulated the problem in F-CIT, did so either after seeing a definition of the network flow problem or after seeing both the definition and an example flow problem. Table 23 provides demographic information about the participants categorized based on their performances in addressing the second problem.

Regarding the mistakes made during the study, we first observed that (as expected) the participants made more mistakes when solving the second problem, compared to the other two problems. While the average number of mistakes made for the second problem was 5.06 , those for the first and third problems were 0.50 and 0.67 , respectively.

We then observed a debugging pattern. The participants, solely for the second problem, found expressing the entities as constraints easier than expressing the FCIT models. They, therefore, used the entity constraints to debug the models. More specifically, to gain insight as well as to test their hypotheses, they tended to click on the Test buttons associated with the entity fields. When the results obtained were not expected, they modified and fixed the models.

Another interesting observation we made is that more than half of the participants (especially for the first and third problems) encoded the F-CIT models as they were reading the study descriptions. That is, as they discovered new system constraints (e.g., parameters and their domains), they updated the models, suggesting that they knew what to look for in the requirements to develop the F-CIT models.

Last but not least, Table 24 presents the outcome of the exit survey. Regarding the questions Q1-Q2 (i.e., Q1.a-Q1.c and Q2.a-Q2.d) and Q6-Q8, all of the participants "agreed" or "strongly agreed" that 1) they understood the basic concepts both in constraint solving and in F-CIT, 2) they found F-CIT useful, 3) they would use F-CIT in a project, and 4) they would recommend F-CIT to others.

Regarding Q10, one suggestion was to improve the syntax of the language we used for expressing the 
TABLE 24: Responses to the exit survey given in Table 22.

\begin{tabular}{|l|c|c|c|c|c|}
\hline & $\begin{array}{c}\text { strongly } \\
\text { disagree }\end{array}$ & disagree & neutral & agree & $\begin{array}{c}\text { strongly } \\
\text { agree }\end{array}$ \\
\hline Q1a & 0 & 0 & 0 & 2 & 18 \\
Q1b & 0 & 0 & 0 & 3 & 17 \\
Q1c & 0 & 0 & 0 & 4 & 16 \\
Q2a & 0 & 0 & 0 & 3 & 17 \\
Q2b & 0 & 0 & 0 & 6 & 14 \\
Q2c & 0 & 0 & 0 & 4 & 16 \\
Q2d & 0 & 0 & 0 & 7 & 13 \\
Q6 & 0 & 0 & 0 & 7 & 13 \\
Q7 & 0 & 0 & 0 & 7 & 13 \\
Q8 & 0 & 0 & 0 & 4 & 16 \\
\hline \hline & very & & & & very \\
& difficult & difficult & normal & easy & easy \\
\hline Q3a & 0 & 0 & 1 & 3 & 16 \\
Q3b & 0 & 0 & 1 & 4 & 15 \\
Q4a & 1 & 12 & 7 & 0 & 0 \\
Q4b & 1 & 6 & 9 & 4 & 0 \\
Q5a & 0 & 0 & 0 & 2 & 18 \\
Q5b & 0 & 0 & 0 & 2 & 18 \\
\hline
\end{tabular}

constraints in a way that closely resembles the Boolean expressions used in main stream programming languages. Another suggestion was to develop a means of expressing "long and repetitive" constraints in a more efficient and effective manner, which, in turn, can further simplify the processes of developing the constraints.

\subsection{Discussion}

We observed that some problems are more difficult to formulate in F-CIT than others. This is, indeed, to be expected. After all, solving some problems may require specific background knowledge and not everybody may possess it. Note, however, that the proposed approach still allows experts to formulate such problems in F-CIT and others to use the existing formulations to compute F-CIT objects. For example, the F-CIT formulation we developed to express reachability in graphbased models, can be used to obtain full coverage under other reachability-based coverage criteria (other than the ones we studied in this work) by changing the entities to be covered.

\section{General Discussion}

In this section, we informally discuss the proposed approach in an attempt to 1) address the additional questions that the reader may have, 2) discuss the big picture, in which we envision F-CIT to be an integral part, and 3) present possible avenues for future research. To further support the discussion, we also provide simple examples and code segments as we see fit.

F-CIT is not a methodology for choosing the entities to be tested. It rather takes as input a set of testable entities and aims to find a minimum number of test cases, such that every required entity is covered by at least one test case.

In the absence of a methodology or a tool that can automatically determine what needs to be tested, such as the existing structural code coverage criterion we used in Section 5.1, identifying the set of entities to be tested may require some effort. Note, however, that if the entities at question should really be tested, then they, in one way or another, must be defined and enumerated regardless of whether F-CIT or a specialized constructor is used.

Once the entities are determined, one may consider developing a specialized CIT constructor. To do that, however, a procedure, which determines whether a given set of entities can be covered together in a test case or not, needs to be devised. But, then, the very same procedure can be used as the "solver" in FCIT, which in turn offers a constructor for free. Note that, as we have discussed in Section 4.5, given such a procedure, the entities can be represented in any form desired (e.g., not necessarily in formal logic), since FCIT does not need to interpret them.

After all, developing specialized CIT constructors may not be easy. As a matter of fact, we introduced our generate-and-cover constructor (Algorithm 2) to mimic one of the simplest ways of generating CIT objects: Keep on randomly generating valid test cases until all the required entities have been covered. However, developing a high-performing specialized constructor is quite challenging, which is also apparent from more than 50 papers published in the literature, the sole purpose of which is to compute standard covering arrays [2]. Therefore, our goal is to generalize the construction as much as possible, so that the collective effort spent for developing F-CIT constructors can be leveraged in a wider spectrum of test scenarios, which in turn increases the flexibility of CIT.

We believe that the performance (i.e., the construction times) of F-CIT constructors can further be improved by using "hints." The idea behind using hints stems from a simple observation of ours: Testable entities to be covered are typically composed of the same set of sub-entities, e.g., the same conjuncts appear in multiple testable entities. Therefore, in the processes of computing F-CIT objects, the same constraints are often solved multiple times. Consequently, capturing the relationships between these recurring constraints (i.e., sub-entities) in the form of hints can improve the efficiency of F-CIT constructors by reducing the number of times the solver is called and/or by calling the solver with simpler constraints. For example, if it is known that the 2-orders $\left[v_{1}, v_{2}\right]$ and $\left[v_{2}, v_{3}\right]$ are covered by a test case for a DAG, then it can be inferred without even using a solver that the 2-order $\left[v_{1}, v_{3}\right]$ as well as the 3 -order $\left[v_{1}, v_{2}, v_{3}\right]$ are also covered by the same test case. We have indeed been working on expressing hints 
in a way (e.g., as constraints), such that they can be leveraged regardless of the F-CIT constructor in use. Although initial feasibility studies show promise, the aforementioned work is still at its early stages.

We also believe that tools (e.g., front-ends) that can provide various means for defining the coverage criteria as well as the input spaces, such that the testable entities selected by the coverage criteria are automatically generated, can be of further practical use. The important point to note, however, is that regardless of the way these tools operate, the cov-CSP problem, thus, the F-CIT constructors we have developed in this work, will stay intact.

To this end, we make two observations. First, declarative modeling approaches, such as the Answer Set Programming (ASP) approach we used in Section 5.2, is a good fit for F-CIT, as they express the logic of a computation without describing its control flow. Second, these approaches can further leverage the commonalities in the coverage criteria and the test spaces that may be present in different application domains. For example, the test spaces we have used in all of our three studies (Sections 5.1-5.3) can indeed be expressed by using a single DAG-based model. In such a model, a node can be associated with a possibly empty set of configuration options, each of which takes its value from a discrete domain. And, an edge can be associated with a condition, which needs to be satisfied before the edge can be taken.

With regard to Study 2. We, indeed, used such DAG-based models in Study 2 (Section 5.2). Since the only thing that mattered in that study was the possible orderings of nodes, no configuration option was associated with the nodes and all the edge conditions were true.

With regard to Study 1 . The virtual options in Study 1 (Section 5.1) can also be modeled by using DAGs. More specifically, each virtual option can be expressed by using the control flow graph (CFG) of the respective if-then-else directive, in which an edge originating from a decision node is associated with a condition that specifies the respective outcome of the decision. For example, one of the edges that originates from the decision if (c) then $\{.$.$\} else\{...\}$ would be associated with $c$, representing the then branch, and the other edge would be associated with $\neg \mathrm{c}$, representing the else branch. Then, covering the virtual settings of a virtual option is the same as making sure that all the edges originating from the decision nodes in the CFG are covered. That is, each edge originating from a decision node corresponds to a virtual setting, which is expressed as the conjunction of all the conditions on the path from the entry node to the destination node of the edge.

To demonstrate the proposed approach, we have extended the ASP encoding given in Figure 5. Figure 9

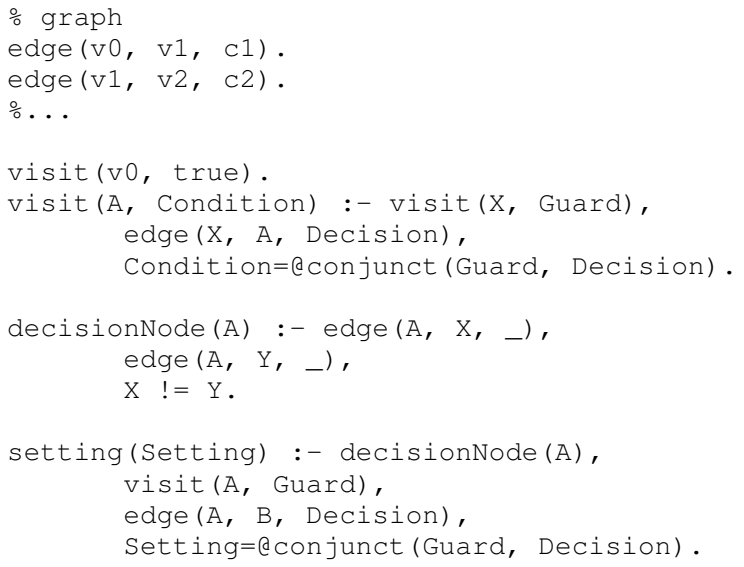

Fig. 9: An ASP encoding for determining the virtual settings of a virtual option expressed as a control flow graph, in which the edges originating from a decision are associated with the conditions required to take these edges.

presents the extended encoding. The first thing we did was to add another parameter to edge ( . . ) to express the conditions associated with edges. More specifically, a fact edge ( $A, B$, Condition) now indicates that there is an edge from $A$ to $B$, which can only be taken if the condition Condition holds. Note that the encoding in Figure 5 can be made compatible with the new encoding by replacing all the edge $(A, B)$ facts by edge ( $A, B$, true), indicating that all edge conditions are true.

Given the control flow graph of a virtual option, in which the entry node is $\mathrm{v} 0$ and the unconditional edges are associated with true, a decision node $A$ is a node, from which at least two edges originate (i.e., a node with an out-degree of at least 2):

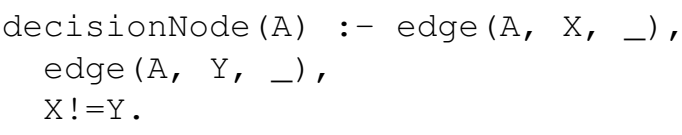

The condition Condition required to visit node $A$ starting from $\mathrm{v} 0$ is the conjunct of the condition Guard, which is required to visit the preceding node $X$, and the condition Decision, which is associated with the edge from $\mathrm{X}$ to $\mathrm{A}$ :

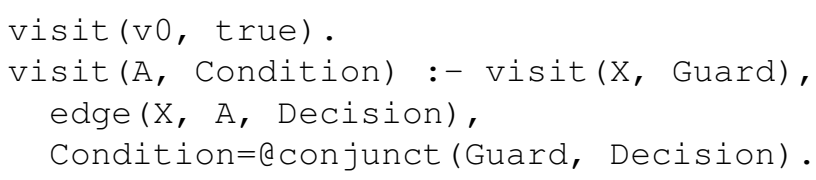

For every edge originating from a decision node, the condition required to take the edge then becomes a virtual setting:

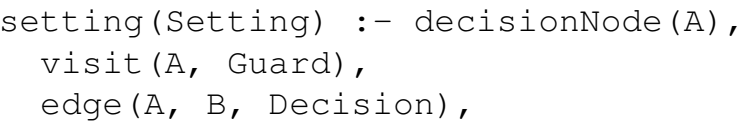


o graph

node (v0, o1, 0).

node (v0, o1, 1).

node (v0, o2, 0$)$.

node (v0, o2, 1).

$\circ$...

tuple(O1, S1, O2, S2) :- node(v0, o1, S1),

node (v0, $02, \mathrm{~S} 2)$,

$\mathrm{O} 1<\mathrm{O} 2$.

Fig. 10: An ASP encoding enumerating all 2-tuples.

Setting=@conjunct (Guard, Decision) .

Note that this encoding is not concerned with solving the conditions, which will indeed be carried out by the F-CIT constructor. It rather forms the virtual settings by joining the individual edge conditions, each of which is expressed as a Boolean expression. Therefore, the edge conditions can be as complex as possible and be processed in any format desired, e.g., as strings. Furthermore, since virtual settings will be Boolean expressions by construction, they can directly be used by our F-CIT constructors without any further processing.

With regard to Study 3. Last but not least, the space of all valid configurations (thus, the space of all valid $t$-tuples) in Study 3 can also be expressed by using the same DAG-based modeling approach. To this end, all we need is a single node, with which all the configuration options of the subject application under test are associated. Since all possible combinations of option settings were valid in Study 3, there is no need to leverage the reachability constraints that can be provided by a graph-based model. The space of all valid $t$-tuples can then be expressed as all possible combinations of settings for $t$ distinct options.

To demonstrate the proposed approach, we have extended the ASP encoding given in Figure 5, such that each node can be associated with a set of configuration options, each of which can take its value from a discrete domain. All the configuration options of the system under test can then be associated with a single node, e.g., the entry node $v 0$.

Figure 10 presents an example encoding for enumerating 2-tuples. In this encoding, the node $v 0$ has two binary options, namely $\circ 1$ and $\circ 2$, each of which can take on either 0 or 1 . All possible 2-tuples for this model can then be enumerated by using:

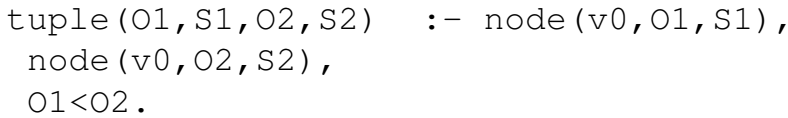

Note that the constraint $\mathrm{O} 1<02$ above is used to put the configuration options in an order, so that only distinct tuples are generated. The tuple weights can then be computed as described in Section 5.3.
Note further that both of the encodings given in Figures 9 and 10 can trivially be extended for different coverage strengths.

\section{ThREATS to Validity}

All empirical studies suffer from threats to their internal and external validity. For this work, we were primarily concerned with threats to external validity since they limit our ability to generalize the results of our studies to industrial practice.

One threat concerns the representativeness of the case studies as well as the subject applications used in the experiments. To alleviate this issue, we addressed a different CIT problem in each case study.

In the first study (Section 5.1), we enhanced standard CIT with a well-known structural code coverage metric, namely decision coverage, and conducted comparative studies on 12 well-known software systems, including Python, vim, and xterm. In the second study (Section 5.2), we enhanced a number of existing order-based coverage criteria. In the third study (Section 5.3), we developed solutions for a problem faced by a successful consumer electronics company and evaluated the proposed approach by using the data collected from the field. Furthermore, not only the CIT problems we have addressed, but also the solution approaches we have developed were diverse. In the first study (Section 5.1), the values of the parameters were Boolean constraints, rather than discrete values, and we used F-CIT with a SAT solver. In the second study (Section 5.2), we expressed the reachability problem in DAGs as a constraint satisfaction problem (CSP) and used a CSP solver. In the third study (Section 5.3), we worked with parameters, each of which takes on a value from a discrete set of values and used F-CIT with a simple, application-specific constraint solver.

We have, however, not directly studied the faultdetection abilities of the F-CIT objects we computed. In the first study (Section 5.1), we developed F-CIT objects to obtain full decision coverage. The decision coverage criterion is, indeed, a well-known structural code coverage criterion for measuring the adequacy of test suites. Therefore, its fault-detection abilities are well-studied [58]. In the second study (Section 5.2), we enhanced a number of existing order-based coverage criteria, which have already been shown to be effective in testing event-driven systems [9]. In the third study (Section 5.3), we developed usage-based CIT objects to reduce the size of the interaction test suites by covering only the $t$-tuples (or a fraction of them) seen in the field. Consequently, for the test scenarios, in which standard covering arrays are infeasible (or undesirable) due to their sizes, usage-based CIT objects would offer the same (or similar) fault revealing abilities for the faults caused by the $t$-tuples seen in the field. 
The number of times we repeated the experiments in the paper varied depending on the cost of the respective experiments. We, however, opted to work on larger CIT problems with smaller repetition counts, rather than working on smaller formulations with larger repetition counts. Furthermore, for each study, we have added a discussion for the costs involved in the study. The actual costs, however, may vary depending on the experience of the tester.

Regarding the user studies, all the participants in these studies were students. We, however, had both undergraduate- and graduate-level students with some background on software testing. Furthermore, more than half of these students had taken at least one course where standard CIT approaches were studied. A related threat concerns the representativeness of the problems used in the user studies. We, however, used the smaller instances of the very same problems we studied in this work (Sections 5.1-5.3). Furthermore, these problems were not known to the participants before taking part in the study. The participants were asked to finish working on one problem before moving to the subsequent problem. They were also required to finish all the studies in a single session. Had they been given more time and/or more instances of the same problems, more participants might have successfully formulated them in a generalizable manner and/or identified and fixed the issues with their formulations.

\section{Related Work}

The basic justification for using standard $t$-way covering arrays is that they can (under certain assumptions) reveal the failures caused by the interactions of $t$ or fewer parameters. As a matter of fact, the results of many empirical studies suggest that a majority of parameter-related failures in practice are often caused by the interactions of only a small number of parameters [59], [2], [60], [61]. That is, $t$ is generally much smaller than the number of parameters, typically $2 \leq t \leq 6$ with $t=2$ (i.e., pairwise testing) being the most common case [3]. Furthermore, for a fixed $t$, as the input space grows (e.g., as the number of parameters increases), the size of a covering array represents an increasingly smaller proportion of the whole input space. Thus, covering arrays can efficiently handle very large input spaces.

Computing standard covering arrays is an NP-hard problem [62]. Standard covering array constructors can be divided into four broad categories based on the construction approach they employ: random searchbased constructors [60], [63], greedy constructors [3], [64], [65], metaheuristic search-based constructors [66], [67], [68], [69], [70], [71], and mathematical constructors [72], [73]. Our work is different in that, while these constructors compute standard covering arrays, the constructors we have presented in this paper compute
F-CIT objects, of which the standard covering arrays are a special instance, by solving the cov-CSP problem.

Constraints in combinatorial interaction testing have also been of practical [15] and theoretical interest [23]. Based on their strictness, CIT constraints can be divided into hard constraints and soft constraints. Hard constraints mark combinations of option settings that are not permitted [17]. Soft constraints, on the other hand, mark combinations of option settings that are permitted, but not desirable [16]. In this work, we define two types of constraints: model constraints and constraints representing entities to be covered. The former types of constraints are system-wide hard constraints, whereas the latter types are hard constraints, the scope of which is limited to the test case covering the entities, i.e., only the test case that covers an entity must satisfy the respective constraint.

A relatively recent work [24] discovers the GUI widgets and Android Activities that interact with each other, by using static analysis and uses this information together with a constraint solver to reduce the number of combinations (thus the number of test cases) required for testing mobile applications. Our work is different in that the aforementioned work uses constraint solving (in a flexible manner by using Alloy [74]) solely to enumerate the testable entities to be covered, which in this context correspond to sequences of Android Activities that can be visited (e.g., prime paths in the activity transition graph of the application under test [24]) and/or the combinations of values that can be tested for the interacting GUI widgets. Once these entities are enumerated, the actual test cases are generated by first computing the Cartesian product of the values for the interacting GUI widgets in each Activity and then by computing the Cartesian product of the test cases generated for the interacting Activities. FCIT, on the other hand, takes the entities to be covered as input and uses constraint solving for generating test cases to cover all the required entities. And, it does so by generalizing the construction of CIT objects in the form of the cov-CSP problem. From this perspective, F-CIT complements the aforementioned work (rather than replacing it) because once the testable entities are enumerated in [24], F-CIT can be used to generate the test cases. Furthermore, the aforementioned work [24] does not concern about generalizing the construction of CIT objects, which is, indeed, the main concern addressed in our work. For example, it is not clear at all whether the construction approach in [24] can be used to compute the different types of F-CIT objects introduced in this work (Sections 5.1-5.3).

Seeding has also been frequently used in combinatorial interaction testing [2]. Some example uses can be summarized as follows: 1) to guarantee the inclusion of certain configurations by having them in the seed; 2) to reduce the cost of testing by including already 
tested configurations in the seed; and 3) for incremental construction of covering arrays by using lower strength covering arrays as seeds to compute higher strength covering arrays [30], [75]. In this work, we have developed a seeding mechanism for F-CIT and used it in two different ways: 1) to combine multiple coverage criteria and 2) to incrementally construct F-CIT objects.

\section{Concluding Remarks}

In this work, we have first presented F-CIT to make combinatorial interaction testing more flexible. In FCIT, both the testable entities to be covered and the space of test cases, from which the samples will be drawn, are expressed as constraints. Consequently, the problem of computing F-CIT objects, turns into an interesting constraint solving problem, which we call cov-CSP. Given a set of constraints, each representing a testable entity to be covered, cov-CSP aims to divide the set into a minimum number of satisfiable clusters, such that a solution for a cluster represents a test case, covering the testable entities included in the cluster. The collection of all the test cases computed for the clusters constitute the F-CIT object, covering each required testable entity at least once.

We have then developed two constructors, namely cover-and-generate and generate-and-cover, to solve the cov-CSP problem, thus to compute F-CIT objects. These constructors can work with any types of constraints as long as an appropriate solver, the purpose of which is to determine whether a given set of entities can be covered in a single test case or not, is provided.

To evaluate F-CIT, we have first carried out three case studies, each of which focused on a different CIT problem, demonstrating that F-CIT is more flexible than the existing CIT approaches. We have arrived at this conclusion by noting that, in these studies, it was either unclear how to use the existing constructors (if at all possible) to compute the requested CIT objects, or the existing constructors required nontrivial modifications or excessive number of test cases to guarantee full coverage. F-CIT, on the other hand, used the same F-CIT constructor to compute all the requested CIT objects these studies without requiring any modifications.

We have also carried out user studies to further evaluate F-CIT, demonstrating the usability of the proposed approach. One thing we observed in these studies is that some problems are more difficult to formulate in F-CIT than others as they require some specific background knowledge, which may not be possessed by everybody. To alleviate these issues, we are developing declarative modeling-based front-ends to flexibly define both the coverage criteria to be used and the test spaces, from which the samples are drawn.

Another avenue for future work is to develop alternative approaches for solving the cov-CSP problem, i.e., developing better constructors. Yet another avenue is to capture the domain knowledge in the form of "hints", which are also expressed as constraints, to develop even better constructors. We are also interested in demonstrating the flexibility of F-CIT in different application domains.

\section{ACKNOWLEDGments}

This research was supported by the Scientific and Technological Research Council of Turkey (118E204).

\section{REFERENCES}

[1] C. Yilmaz, S. Fouche, M. B. Cohen, A. Porter, G. Demiroz, and U. Koc, "Moving forward with combinatorial interaction testing," Computer, vol. 47, no. 2, pp. 37-45, 2014.

[2] C. Nie and H. Leung, "A survey of combinatorial testing," ACM Computing Surveys (CSUR), vol. 43, no. 2, p. 11, 2011.

[3] D. M. Cohen, S. R. Dalal, M. L. Fredman, and G. C. Patton, "The AETG system: an approach to testing based on combinatorial design," IEEE Trans. on Soft. Eng., vol. 23, no. 7, pp. 437-44, 1997.

[4] A. W. Williams and R. L. Probert, "A practical strategy for testing pair-wise coverage of network interfaces," in Proceedings of Seventh International Symposium on Software Reliability Engineering. IEEE, 1996, pp. 246-254.

[5] P. J. Schroeder, P. Faherty, and B. Korel, "Generating expected results for automated black-box testing," in In Proceedings of the 17th IEEE International Conference on Automated Software Engineering, ASE 2002. IEEE, 2002, pp. 139-148.

[6] C. Yilmaz, M. B. Cohen, and A. A. Porter, "Covering arrays for efficient fault characterization in complex configuration spaces," IEEE Trans. Software Eng., vol. 32, no. 1, pp. 20-34, 2006.

[7] M. F. Johansen, Ø. Haugen, and F. Fleurey, "An algorithm for generating $\mathrm{t}$-wise covering arrays from large feature models," in Proceedings of the 16th International Software Product Line Conference-Volume 1. ACM, 2012, pp. 46-55.

[8] Y. Lei, R. H. Carver, R. Kacker, and D. Kung, "A combinatorial testing strategy for concurrent programs," Software Testing, Verification and Reliability, vol. 17, no. 4, pp. 207-225, 2007.

[9] X. Yuan, M. B. Cohen, and A. M. Memon, "GUI interaction testing: Incorporating event context," IEEE Transactions on Software Engineering, vol. 37, no. 4, pp. 559-574, 2011.

[10] C. Yilmaz, "Test case-aware combinatorial interaction testing," IEEE Transactions on Software Engineering, vol. 39, no. 5, pp. 684706, 2013.

[11] G. Demiroz and C. Yilmaz, "Cost-aware combinatorial interaction testing," in Proceedings of the Internatinoal Conference on Advances in System Testing and Validation Lifecycles, 2012, pp. 916.

[12] A. Javeed and C. Yilmaz, "Combinatorial interaction testing of tangled configuration options," in 2015 IEEE Eight International Conference on Software Testing, Verification and Validation Workshops (ICSTW). IEEE, 2015, pp. 1-4.

[13] J. Lawrence, R. N. Kacker, Y. Lei, D. R. Kuhn, and M. Forbes, "A survey of binary covering arrays," the electronic journal of combinatorics, vol. 18, no. 1, p. P84, 2011.

[14] E. Dumlu, C. Yilmaz, M. B. Cohen, and A. Porter, "Feedback driven adaptive combinatorial testing," in Proceedings of the International Symposium on Software Testing and Analysis. ACM, 2011, pp. 243-253.

[15] M. B. Cohen, M. B. Dwyer, and J. Shi, "Interaction testing of highly-configurable systems in the presence of constraints," in Proceedings of the 2007 International Symposium on Software Testing and Analysis, 2007, pp. 129-139.

[16] R. C. Bryce and C. J. Colbourn, "Prioritized interaction testing for pair-wise coverage with seeding and constraints," Information and Software Technology, vol. 48, no. 10, pp. 960 - 970, 2006, advances in Model-based Testing. 
[17] G. Mats, O. Jeff, and M. Jonas, "Handling constraints in the input space when using combination strategies for software testing," University of Skvde, School of Humanities and Informatics, Tech. Rep. HS- IKI -TR-06-001, 2006.

[18] C. Yilmaz, "Test case-aware combinatorial interaction testing," IEEE Transactions on Software Engineering, vol. 39, no. 5, pp. 684706, 2013.

[19] M. Makaś, "A certain version of preservationism," Logic and Logical Philosophy, vol. 26, no. 1, pp. 63-77, 2016.

[20] P. K. Schotch and R. E. Jennings, "Inference and necessity," Journal of Philosophical Logic, vol. 9, no. 3, pp. 327-340, 1980.

[21] N. Rescher and R. Manor, "On inference from inconsistent premisses," Theory and decision, vol. 1, no. 2, pp. 179-217, 1970.

[22] A. Biere, M. Heule, and H. van Maaren, Handbook of satisfiability. IOS press, 2009, vol. 185

[23] P. Danziger, E. Mendelsohn, L. Moura, and B. Stevens, "Covering arrays avoiding forbidden edges," Theoretical Computer Science, vol. 410, no. 52, pp. 5403-5414, 2009.

[24] N. Mirzaei, J. Garcia, H. Bagheri, A. Sadeghi, and S. Malek, "Reducing combinatorics in gui testing of android applications," in Proceedings of the 38th International Conference on Software Engineering, ser. ICSE $16 . \quad$ New York, NY, USA: Association for Computing Machinery, 2016, p. 559570.

[25] H. Mercan and C. Yilmaz, "A constraint solving problem towards unified combinatorial interaction testing," in Proceedings of the 7th Workshop on Constraint Solvers in Testing, Verification, and Analysis, vol. 1639, no. 5. CEUR, 2016, pp. 24-30.

[26] A. Javeed, "Gray-box combinatorial interaction testing," Master's thesis, 2015.

[27] Y. T. Yu and M. F. Lau, "A comparison of $\mathrm{mc} / \mathrm{dc}$, mumcut and several other coverage criteria for logical decisions," Journal of Systems and Software, vol. 79, no. 5, pp. 577-590, 2006.

[28] "Radon." [Online]. Available: https://radon.readthedocs.io/ en/latest/

[29] L. Yu, Y. Lei, R. N. Kacker, and D. R. Kuhn, "ACTS: A combinatorial test generation tool," in 2013 IEEE Sixth International Conference on Software Testing, Verification and Validation (ICST). IEEE, 2013, pp. 370-375

[30] S. Fouché, M. B. Cohen, and A. Porter, "Incremental covering array failure characterization in large configuration spaces," in Proceedings of the eighteenth international symposium on Software testing and analysis. ACM, 2009, pp. 177-188.

[31] J. Liebig, S. Apel, C. Lengauer, C. Kästner, and M. Schulze, “An analysis of the variability in forty preprocessor-based software product lines," in Proceedings of the 32nd ACM/IEEE International Conference on Software Engineering-Volume 1. ACM, 2010, pp. $105-114$.

[32] "SATisPy." [Online]. Available: https://github.com/netom/ satispy

[33] N. Eén and N. Sörensson, "An extensible SAT-solver," in International conference on theory and applications of satisfiability testing. Springer, 2003, pp. 502-518.

[34] P. Samuel and A. T. Joseph, "Test sequence generation from uml sequence diagrams," in Ninth ACIS International Conference on Software Engineering, Artificial Intelligence, Networking, and Parallel/Distributed Computing, 2008. SNPD'08. IEEE, 2008, pp. 879-887.

[35] F. Belli, M. Beyazit, and N. Güler, "Event-Based GUI testing and reliability assessment techniques-an experimental insight and preliminary results," in IEEE Fourth International Conference on Software Testing, Verification and Validation Workshops (ICSTW). IEEE, 2011, pp. 212-221.

[36] A. M. Memon, "An event-flow model of GUI-based applications for testing," Software testing, verification and reliability, vol. 17, no. 3, pp. 137-157, 2007.

[37] M. Ç. Çalpur, "Interleaving coverage criteria oriented testing of multithreaded applications," Master's thesis, 2012.

[38] D. L. Bruening, "Systematic testing of multithreaded Java programs," Ph.D. dissertation, Massachusetts Institute of Technology, 1999.

[39] M. Musuvathi, S. Qadeer, T. Ball, M. Musuvathi, S. Qadeer, and T. Ball, "Chess: A systematic testing tool for concurrent software," Technical Report MSR-TR-2007-149, Microsoft Research, Tech. Rep., 2007.
[40] S. Lu, W. Jiang, and Y. Zhou, "A study of interleaving coverage criteria," in the 6th joint meeting on European software engineering conference and the ACM SIGSOFT symposium on the foundations of software engineering: companion papers. ACM, 2007, pp. 533-536.

[41] D. R. Kuhn, J. M. Higdon, J. F. Lawrence, R. N. Kacker, and Y. Lei, "Combinatorial methods for event sequence testing," in Proceedings of the 5th IEEE International Conference on Software Testing, Verification and Validation, 2012, pp. 601-609.

[42] "Apache ActiveMQ." [Online]. Available: http://activemq. apache.org/

[43] A. Biere, A. Cimatti, E. M. Clarke, O. Strichman, Y. Zhu et al., "Bounded model checking." Advances in computers, vol. 58, no. 11, pp. 117-148, 2003.

[44] R. Ahlswede, N. Cai, S.-Y. Li, and R. W. Yeung, "Network information flow," IEEE Transactions on information theory, vol. 46, no. 4, pp. 1204-1216, 2000.

[45] J. E. Hopcroft, Introduction to Automata Theory, Languages and Computation: For VTU, 3/e. Pearson Education India, 2013.

[46] S. Lombardy, Y. Régis-Gianas, and J. Sakarovitch, "Introducing vaucanson," Theoretical Computer Science, vol. 328, no. 1-2, pp. 77-96, 2004

[47] V. W. Marek and M. Truszczyński, "Stable models and an alternative logic programming paradigm," in The Logic Programming Paradigm. Springer, 1999, pp. 375-398.

[48] I. Niemelä, "Logic programs with stable model semantics as a constraint programming paradigm," Annals of Mathematics and Artificial Intelligence, vol. 25, no. 3-4, pp. 241-273, 1999.

[49] T. Eiter, G. Ianni, and T. Krennwallner, "Answer set programming: A primer," in Reasoning Web International Summer School. Springer, 2009, pp. 40-110.

[50] C. Baral, Knowledge representation, reasoning and declarative problem solving. Cambridge university press, 2003.

[51] N. Tamura and M. Banbara, "Sugar: A CSP to SAT translator based on order encoding," Proceedings of the Second International CSP Solver Competition, pp. 65-69, 2008.

[52] B. Jenkins, "jenny: A pairwise testing tool," http://www. burtleburtle.net/bob/index.html, 2005.

[53] J. Czerwonka, "Pairwise testing in the real world: Practical extensions to test-case scenarios," Microsoft Corporation, Software Testing Technical Articles, 2008.

[54] H. Katebi, K. Sakallah, and J. Marques-Silva, "Empirical study of the anatomy of modern sat solvers," Theory and Applications of Satisfiability Testing-SAT 2011, pp. 343-356, 2011.

[55] L. M. de Moura and N. Bjørner, "Satisfiability modulo theories: An appetizer," SBMF, vol. 5902, pp. 23-36, 2009.

[56] M. B. Cohen, P. B. Gibbons, W. B. Mugridge, C. J. Colbourn, and J. S. Collofello, "A variable strength interaction testing of components," in Proceedings of 27th IEEE Annual International Computer Software and Applications Conference, (COMPSAC). IEEE, 2003, pp. 413-418.

[57] M. S. Bazaraa, J. J. Jarvis, and H. D. Sherali, Linear programming and network flows. John Wiley \& Sons, 2011.

[58] X. Cai and M. R. Lyu, "The effect of code coverage on fault detection under different testing profiles," ACM SIGSOFT software engineering notes, vol. 30, no. 4, pp. 1-7, 2005

[59] R. Kuhn, Y. Lei, and R. Kacker, "Practical combinatorial testing: Beyond pairwise," It Professional, vol. 10, no. 3, 2008.

[60] P. J. Schroeder, P. Bolaki, and V. Gopu, "Comparing the fault detection effectiveness of n-way and random test suites," in Proc. of the 2004 Int'l Symp. on Empirical Software Engineering, 2004, pp. 49-59.

[61] D. R. Kuhn and V. Okum, "Pseudo-exhaustive testing for software," in 30th Annual IEEE/NASA Software Engineering Workshop. SEW'06. IEEE, 2006, pp. 153-158.

[62] E. Maltais and L. Moura, "Hardness results for covering arrays avoiding forbidden edges and error-locating arrays," Theoretical Computer Science, vol. 412, no. 46, pp. 6517-6530, 2011.

[63] R. Huang, X. Xie, T. Y. Chen, and Y. Lu, "Adaptive random test case generation for combinatorial testing," in Proceedings of 36 th IEEE Annual International Computer Software and Applications Conference, (COMPSAC). IEEE, 2012, pp. 52-61.

[64] Y. Lei, R. Kacker, D. R. Kuhn, V. Okun, and J. Lawrence, "IPOG/IPOG-D: efficient test generation for multi-way combinatorial testing," Softw. Test. Verif. Reliab., vol. 18, pp. 125-148, September 2008. 
[65] Z. Wang and H. He, "Generating variable strength covering array for combinatorial software testing with greedy strategy." JSW, vol. 8, no. 12, pp. 3173-3181, 2013.

[66] M. B. Cohen, P. B. Gibbons, W. B. Mugridge, and C. J. Colbourn, "Constructing test suites for interaction testing," in Proceedings of the 25th International Conference on Software Engineering, 2003, pp. $38-48$.

[67] R. C. Bryce and C. J. Colbourn, "One-test-at-a-time heuristic search for interaction test suites," in Proceedings of the 9th annual conference on Genetic and evolutionary computation. New York, NY, USA: ACM, 2007, pp. 1082-1089.

[68] H. Wu, C. Nie, F.-C. Kuo, H. Leung, and C. J. Colbourn, "A discrete particle swarm optimization for covering array generation," IEEE Transactions on Evolutionary Computation, vol. 19, no. 4, pp. 575-591, 2015.

[69] P. Galinier, S. Kpodjedo, and G. Antoniol, "A penalty-based Tabu search for constrained covering arrays," in Proceedings of the Genetic and Evolutionary Computation Conference. ACM, 2017, pp. 1288-1294.

[70] Y. Jia, M. B. Cohen, M. Harman, and J. Petke, "Learning combinatorial interaction test generation strategies using hyperheuristic search," in Proceedings of the 37th IEEE International Conference on Software Engineering (ICSE), vol. 1. IEEE, 2015, pp. $540-550$.

[71] J. Torres-Jimenez and E. Rodriguez-Tello, "New bounds for binary covering arrays using simulated annealing," Information Sciences, vol. 185, no. 1, pp. 137-152, 2012.

[72] N. Kobayashi, "Design and evaluation of automatic test generation strategies for functional testing of software," Ph.D. dissertation, Osaka University, Osaka, Japan, 2002.

[73] C. J. Colbourn, "Combinatorial aspects of covering arrays," Le Matematiche (Catania), vol. 58, no. 121-167, pp. 0-10, 2004.

[74] D. Jackson, "Alloy: a lightweight object modelling notation," ACM Transactions on Software Engineering and Methodology (TOSEM), vol. 11, no. 2, pp. 256-290, 2002.

[75] C. Yilmaz, E. Dumlu, M. B. Cohen, and A. Porter, "Reducing masking effects in combinatorial interaction testing: A feedback driven adaptive approach," IEEE Transactions on Software Engineering, vol. 40, no. 1, pp. 43-66, 2014.

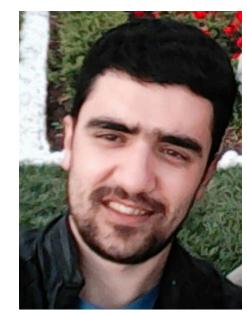

Hanefi Mercan received the BS degree in mathematics and the MS degree in computer science in 2012 and 2015, respectively. He is currently a PhD student in Sabanci University. His research interests include software testing and parallel computing.

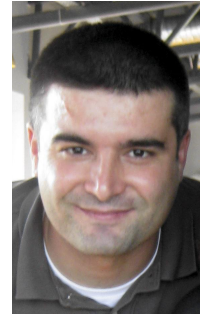

Cemal Yilmaz received the BS and MS degrees in computer engineering and information science from Bilkent University, Ankara, Turkey, in 1997 and 1999, respectively. In 2005, he received the $\mathrm{PhD}$ degree in computer science from the University of Maryland at College Park. Between 2005 and 2008, he worked as a post-doctoral researcher at IBM Thomas J. Watson Research Center, Hawthorne, New York. He is currently an assistant professor of computer science in the Faculty of Engineering and Natural Sciences, Sabanci University, Istanbul, Turkey. His current research interests include software engineering and software quality assurance.

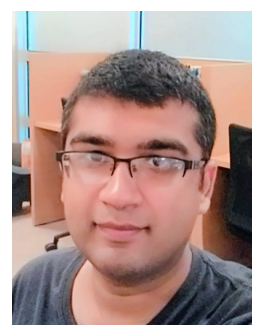

Arsalan Javeed received BS degree in Telecommunication Engineering and $\mathrm{MS}$ in Computer Science and Engineering in 2011 and 2015 respectively. $\mathrm{He}$ is currently a $\mathrm{PhD}$ student at Sabanci University, Turkey. His research interests include Software Engineering, Testing and Security. 